\title{
Cascade Synthesis of 3-Alkylidene Dihydrobenzofuran Derivatives via Rhodium(III)-Catalyzed Redox-Neutral C-H Functionalization/Cyclization
}

Zhi Zhou, Guixia Liu*, Yan Chen and Xiyan Lu*

State Key Laboratory of Organometallic Chemistry, Shanghai Institute of Organic Chemistry, Chinese Academy of Sciences, 345 Lingling Lu, Shanghai, 200032, China E-mail: guixia@sioc.ac.cn; xylu@mail.sioc.ac.cn

\section{Supporting Information}

\section{Contents}

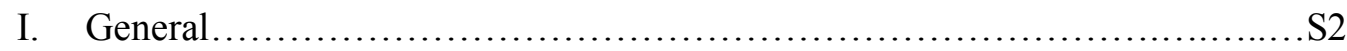

II. Synthesis of tert-butyl Propargyl Carbonates $2 \ldots \ldots \ldots \ldots \ldots \ldots \ldots \ldots \ldots \ldots$. 2

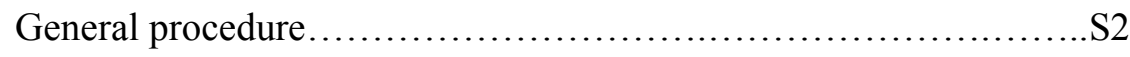

Characterization of tert-butyl Propargyl Carbonates 2.....................S3

III. Synthesis of 3-Alkylidene Dihydrobenzofuran Derivatives..................S5

Optimization Studies........................................... 5

General procedure.........................................S6

Characterization of products...................................................... 7

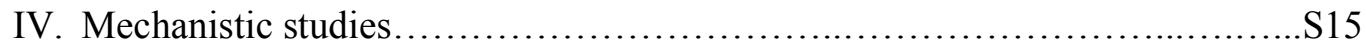

V. Gram Scale Synthesis and Derivatization of 3aa.......................................S20

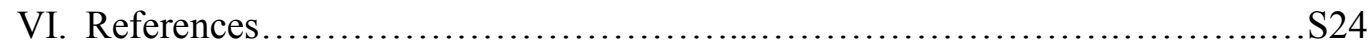

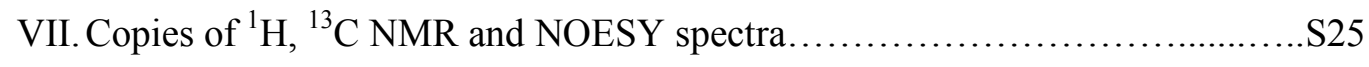




\section{General}

NMR spectra were recorded on a Varian Mercury Vx400 spectrometer in solvents as indicated. Chemical shifts $(\delta)$ are given in ppm relative to TMS. The residual solvent signals were used as references and the chemical shifts converted to the TMS scale $\left(\mathrm{CDCl}_{3}: \delta_{\mathrm{H}}=7.26 \mathrm{ppm}, \delta_{\mathrm{C}}=77.16 \mathrm{ppm} ; \mathrm{d}_{6}-\mathrm{DMSO}: \delta_{\mathrm{H}}=2.50 \mathrm{ppm}, \delta_{\mathrm{C}}=39.52 \mathrm{ppm}\right)$. Infrared spectra were obtained on a Bio-Rad FTS-185 instrument. Mass spectra were provided on Agilent 5973 or Agilent 1100 instruments. All melting points were uncorrected. $\left[\mathrm{Cp}^{*} \mathrm{RhCl}_{2}\right]_{2}$ was purchased from Strem. $N$-phenoxyacetamides were synthesized according to known procedures. ${ }^{1}$ All other reagents were from commercial sources. No attempts were made to optimize yields for substrate synthesis.

\section{Synthesis of tert-butyl Propargyl Carbonates 2}

\section{General Procedure:}

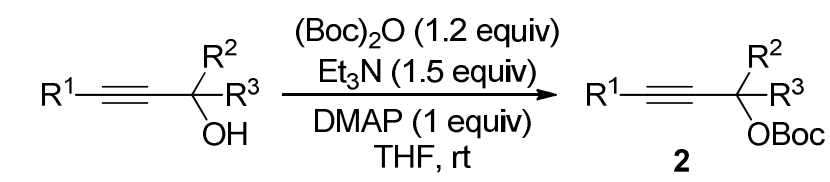

$$
\begin{aligned}
& R^{1}=\text { Phenyl, } R^{2}=R^{3}=H: 2 a ; R^{1}=(4-M e) \text { phenyl, } R^{2}=R^{3}=H: 2 b \\
& R^{1}=(4-M e O) \text { phenyl, } R^{2}=R^{3}=H: 2 c ; R^{1}=\left(4-N_{2}\right) \text { phenyl, } R^{2}=R^{3}=H: 2 d
\end{aligned}
$$$$
R^{1}=(4-F) \text { phenyl, } R^{2}=R^{3}=H: 2 e ; R^{1}=(3-M e) \text { phenyl, } R^{2}=R^{3}=H: 2 f
$$$$
R^{1}=\text { thienyl, } R^{2}=R^{3}=H: 2 g ; R^{1}=\text { Phenyl, } R^{2}=M e, R^{3}=H: 2 i
$$$$
R^{1}=R^{2}=\text { Phenyl, } R^{3}=H: 2 j ; R^{1}=\text { Phenyl, } R^{2}=R^{3}=\text { Me: } 2 k
$$

The alkyne substrates tert-butyl propargyl carbonates $\mathbf{2}$ were prepared following a published procedure ${ }^{2}$ : In a reaction flask, a mixture of (Boc) $)_{2} \mathrm{O}$ (1.2 equiv), $\mathrm{Et}_{3} \mathrm{~N}$ (1.5 equiv) and DMAP (1 equiv) in THF $(0.3 \mathrm{M})$ were stirred at room temperature, followed by dropwise addition of the propargyl alcohol derivative (1 equiv). The reaction was monitored by TLC and then quenched with aqueous $\mathrm{NH}_{4} \mathrm{Cl}$ solution and extracted with $\mathrm{Et}_{2} \mathrm{O}(\mathrm{x} 3)$. The organic phase was washed with brine after which it was dried over $\mathrm{Na}_{2} \mathrm{SO}_{4}$, filtered and evaporated under reduced pressure. The crude product was purified by flash column chromatography on silica gel to give the desired tert-butyl propargyl carbonates $\mathbf{2}$. 


\section{Characterization of tert-butyl Propargyl Carbonates 2:}

2a, $2 \mathbf{a a}, \mathbf{2 a b}, \mathbf{2 a c}, \mathbf{2 h}, \mathbf{2 i}, \mathbf{2} \mathbf{k}$ are known compounds and all data were in agreement with those reported. ${ }^{2,3}$

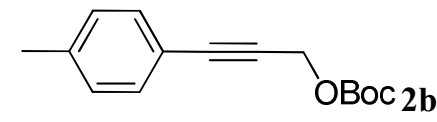

Yield 60\%; light yellow liquid; ${ }^{1} \mathbf{H}$ NMR (400 MHz, $\left.\mathbf{C D C l}_{3}\right): \delta 7.33$ (d, $J=8.0 \mathrm{~Hz}$, 2H), $7.11(\mathrm{~d}, J=8.4 \mathrm{~Hz}, 2 \mathrm{H}), 4.89(\mathrm{~s}, 2 \mathrm{H}), 2.34(\mathrm{~s}, 3 \mathrm{H}), 1.51(\mathrm{~s}, 9 \mathrm{H}) ;{ }^{13}$ C NMR (100 MHz, $\left.\mathbf{C D C l}_{3}\right): \delta 153.0,139.0,131.9,129.2,119.2,87.1,83.0,82.0,55.5,27.9,21.6$; IR (neat, $\mathbf{c m}^{-1}$ ): $v$ 2981, 1740, 1272, 1251, 1157, 1088, 816; MS (m/z, ESI): 264, 208, 129; HRMS calculated for $\mathrm{C}_{15} \mathrm{H}_{22} \mathrm{NO}_{3}\left(\mathrm{M}+\mathrm{NH}_{4}\right)^{+}:$264.1594; Found: 264.1595; $\mathbf{R}_{\mathbf{f}}=0.8(\mathrm{PE}:$ EtOAc, $3: 1)$.

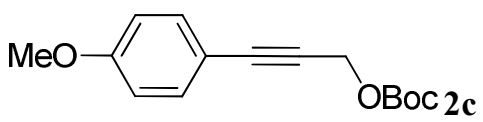

Yield 68\%; white solid; m.p. 56-57 ${ }^{\circ} \mathrm{C} ;{ }^{1} \mathbf{H}$ NMR (400 $\left.\mathbf{M H z}, \mathbf{C D C l}_{3}\right): \delta 7.38$ (d, $J=$ $8.4 \mathrm{~Hz}, 2 \mathrm{H}), 6.83(\mathrm{~d}, J=8.8 \mathrm{~Hz}, 2 \mathrm{H}), 4.89(\mathrm{~s}, 2 \mathrm{H}), 3.80(\mathrm{~s}, 3 \mathrm{H}), 1.51(\mathrm{~s}, 9 \mathrm{H}) ;{ }^{13} \mathrm{C}$ NMR (100 MHz, $\left.\mathbf{C D C l}_{3}\right): \delta$ 160.0, 153.0, 133.5, 114.3, 114.0, 87.0, 82.9, 81.4, 55.5, 55.4, 27.8; IR (neat, $\mathbf{c m}^{-1}$ ): $v$ 2969, 1741, 1512, 1281, 1249, 1149, 837; MS (m/z, EI): 262, 206, 162, 145, 135, 102, 57; HRMS calculated for $\mathrm{C}_{15} \mathrm{H}_{18} \mathrm{O}_{4}(\mathrm{M})^{+}:$: 262.1205; Found: $262.1202 ; \mathbf{R}_{\mathbf{f}}=0.6$ (PE: EtOAc, $\left.3: 1\right)$.

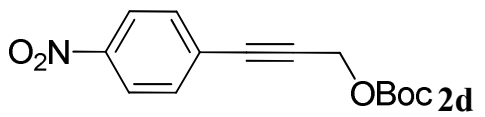

Yield 82\%; yellow solid; m.p. 69-70 ${ }^{\circ} \mathrm{C} ;{ }^{1} \mathbf{H}$ NMR (400 $\left.\mathbf{M H z}, \mathbf{C D C l}_{3}\right): \delta 8.19(\mathrm{~d}, J=$ $8.0 \mathrm{~Hz}, 2 \mathrm{H}), 7.59$ (d, $J=8.4 \mathrm{~Hz}, 2 \mathrm{H}), 4.93$ (s, 2H), 1.52 (s, 9H); ${ }^{13}$ C NMR (100 MHz, $\left.\mathbf{C D C l}_{3}\right): \delta 152.8,147.5,132.7,129.1,123.7,88.1,84.9,83.4,54.9,27.8$; IR (neat, $\left.\mathbf{c m}^{-1}\right):$ 2976, 1741, 1516, 1342, 1276, 1253, 1156, 855; MS (m/z, EI): 277, 221, 160, 130, 102, 57; HRMS calculated for $\mathrm{C}_{14} \mathrm{H}_{15} \mathrm{NO}_{5}(\mathrm{M})^{+}$: 277.0950; Found: $277.0943 ; \mathbf{R}_{\mathbf{f}}=0.7($ PE: EtOAc, $3: 1)$. 


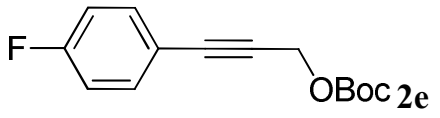

Yield 87\%; yellow liquid; ${ }^{1} \mathbf{H}$ NMR (400 $\left.\mathbf{M H z}, \mathbf{C D C l}_{3}\right): \delta$ 7.45-7.41 (m, 2H), 7.03-6.98 (m, 2H), 4.88 (s, 2H), 1.51 (s, 9H); ${ }^{13} \mathbf{C}$ NMR (100 MHz, CDCl $\left.\mathbf{3}\right): \delta 162.9$ $\left(\mathrm{d}, J_{C-F}=249.0 \mathrm{~Hz}\right), 153.0,133.9\left(\mathrm{~d}, J_{C-F}=8.4 \mathrm{~Hz}\right), 118.3\left(\mathrm{~d}, J_{C-F}=3.0 \mathrm{~Hz}\right), 115.7(\mathrm{~d}$, $\left.J_{C-F}=22.1 \mathrm{~Hz}\right), 85.9,83.1,82.5,55.3,27.8 ;{ }^{19}$ F NMR (376 MHz, DMSO-d 6 ): -145.6; IR (neat, $\left.\mathbf{c m}^{\mathbf{- 1}}\right):$ v 2982, 1744, 1507, 1276, 1254, 1158, 838; MS (m/z, EI): 250, 194, 150, 133, 123, 57; HRMS calculated for $\mathrm{C}_{14} \mathrm{H}_{15} \mathrm{FO}_{3}(\mathrm{M})^{+}:$250.1005; Found: 250.1010; $\mathbf{R}_{\mathbf{f}}=0.8(\mathrm{PE}:$ EtOAc, $3: 1)$.

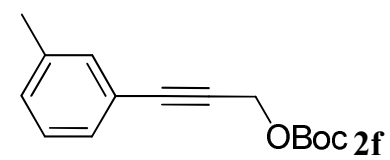

Yield 89\%; colorless liquid; ${ }^{1} \mathbf{H}$ NMR (400 MHz, $\left.\mathbf{C D C l}_{3}\right): \delta$ 7.27-7.24 (m, 2H), 7.19 (t, $J=7.6 \mathrm{~Hz}, 1 \mathrm{H}), 7.13(\mathrm{~d}, J=7.6 \mathrm{~Hz}, 1 \mathrm{H}), 4.89(\mathrm{~s}, 2 \mathrm{H}), 2.32(\mathrm{~s}, 3 \mathrm{H}), 1.51(\mathrm{~s}, 9 \mathrm{H})$; ${ }^{13}$ C NMR (100 MHz, CDCl $)$ ): $\delta$ 153.0, 138.1, 132.5, 129.7, 129.0, 128.3, 122.1, 87.1, 83.0, 82.4, 55.4, 27.9, 21.3; IR (neat, $\mathbf{~ c m}^{-1}$ ): v 2980, 1744, 1371, 1275, 1253, 1159, 1091, 787; MS (m/z, EI): 246, 190, 146, 128, 119, 57; HRMS calculated for $\mathrm{C}_{15} \mathrm{H}_{18} \mathrm{O}_{3}(\mathrm{M})^{+}:$246.1256; Found: 246.1255; $\mathbf{R}_{\mathbf{f}}=0.8$ (PE: EtOAc, 3:1).

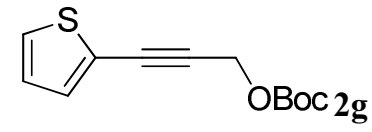

Yield 85\%; yellow liquid; ${ }^{1} \mathbf{H}$ NMR (400 MHz, $\left.\mathbf{C D C l}_{3}\right): \delta$ 7.28-7.23 (m, 2H), 6.96 (m, 1H), 4.91 (s, 2H), 1.51 (s, 9H); ${ }^{13} \mathbf{C}$ NMR (100 MHz, $\left.\mathbf{C D C l}_{3}\right): \delta$ 152.9, 133.1, 128.0, 127.1, 122.1, 86.8, 83.1, 80.4, 55.4, 27.9; IR (neat, $\mathbf{c m}^{-1}$ ): v 2980, 2230, 1744, 1274, 1253, 1160, 1090, 851, 705; MS (m/z, EI): 238, 182, 138, 121, 110, 77, 57; HRMS calculated for $\mathrm{C}_{12} \mathrm{H}_{14} \mathrm{SO}_{3}(\mathrm{M})^{+}:$238.0664; Found: $238.0670 ; \mathbf{R}_{\mathbf{f}}=0.7(\mathrm{PE}$ : EtOAc, 3:1). 


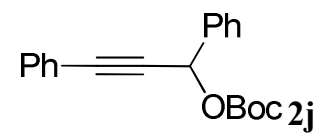

Yield 95\%; yellow solid; m.p. 50-51 ${ }^{\circ} \mathrm{C} ;{ }^{1} \mathbf{H}$ NMR (400 $\left.\mathbf{M H z}, \mathbf{C D C l}_{3}\right): \delta 7.61(\mathrm{~d}, J=$ 8.0 Hz, 2H), 7.48-7.45 (m, 2H), 7.42-7.36 (m, 3H), 7.33-7.28 (m, 3H), 6.49 (s, 1H), $1.50(\mathrm{~s}, 9 \mathrm{H}) ;{ }^{13} \mathbf{C}$ NMR (100 MHz, $\left.\mathbf{C D C l}_{3}\right): \delta$ 152.7, 137.0, 132.0, 129.2, 128.9, 128.8, 128.4, 128.0, 122.3, 87.7, 85.4, 83.1, 69.2, 27.9; IR (neat, cm$^{-1}$ ): v 2984, 1727, 1273, 1246, 1150, 1075, 866, 748, 695; MS (m/z, EI): 252, 208, 191, 179, 165, 130, 57; Anal. calculated for $\mathrm{C}_{20} \mathrm{H}_{20} \mathrm{O}_{3}$ : C, 77.90; H, 6.54; Found: C, 77.86; H, 6.61; $\mathbf{R}_{\mathbf{f}}=$ 0.8 (PE: EtOAc, 3:1).

\section{Synthesis of 3-Alkylidene Dihydrobenzofuran Derivatives Optimization Studies (Table S1)}

Without any particular precautions to extrude oxygen or moisture, the $\mathrm{N}$-phenoxy amide 1 (0.1 mmol, 1 equiv), 3-phenylprop-2-yn-1-yl derivative 2 (0.12 mmol, 1.2 equiv), [Cp* $\left.\mathrm{RhCl}_{2}\right]_{2}(1.6 \mathrm{mg}, 0.0025 \mathrm{mmol}, 2.5 \mathrm{~mol} \%$ ), and additives were weighted in a $4 \mathrm{~mL}$ vial equipped with a stir bar. Solvent $(0.2 \mathrm{M})$ was then added. The reaction was stirred at room temperature for 24 hours under air. Afterwards, it was diluted with EtOAc and transferred to a round bottom flask. Then, the reaction mixture was concentrated and ${ }^{1} \mathrm{H}$ NMR was taken using trimethoxybenzene $(5.6 \mathrm{mg})$ as the internal standard. 
Table S1 Optimization Studies ${ }^{a}$

\begin{tabular}{|c|c|c|c|c|c|}
\hline & & 2 & $\mathrm{OR}^{2} \frac{\begin{array}{c}{\left[\mathrm{Cp}^{*} \mathrm{RhCl}_{2}\right]_{2}(2.5 \mathrm{~m}} \\
\text { additives }\end{array}}{\text { solvent }(0.2 \mathrm{M}), \mathrm{r}}$ & $\%)$ & $\underbrace{\mathrm{NHR}}_{-\mathrm{Ph}}$ \\
\hline Entry & $\mathrm{R}^{1}$ & $\mathrm{R}^{2}$ & Additives (equiv) & Solvent & Yield $^{b}(\%)$ \\
\hline 1 & Ac & $\mathrm{CO}_{2} \mathrm{Me}$ & $\mathrm{CsOAc}(0.5)$ & DCM & 46 \\
\hline 2 & $\mathrm{Ac}$ & $\mathrm{CO}_{2} \mathrm{Me}$ & CsOAc (0.5) & $\mathrm{MeOH}$ & 68 \\
\hline 3 & $\mathrm{Ac}$ & $\mathrm{CO}_{2} \mathrm{Me}$ & $\mathrm{CsOAc}(0.5)$ & dioxane & 22 \\
\hline 4 & $\mathrm{Ac}$ & $\mathrm{CO}_{2} \mathrm{Me}$ & $\mathrm{CsOAc}(0.5)$ & $\mathrm{CH}_{3} \mathrm{CN}$ & 67 \\
\hline 5 & $\mathrm{Ac}$ & $\mathrm{CO}_{2} \mathrm{Me}$ & $\mathrm{CsOAc}(0.5)$ & Toluene & 37 \\
\hline 6 & Ac & $\mathrm{CO}_{2} \mathrm{Me}$ & $\mathrm{CsOAc}(0.5)$ & $\mathrm{EtOH}$ & 54 \\
\hline 7 & Ac & $\mathrm{CO}_{2} \mathrm{Me}$ & $\mathrm{CsOAc}(0.5)$ & ${ }^{i} \mathrm{PrOH}$ & 41 \\
\hline 8 & $\mathrm{Ac}$ & $\mathrm{CO}_{2} \mathrm{Me}$ & $\mathrm{CsOAc}(0.5)$ & ${ }^{t} \mathrm{AmOH}$ & 46 \\
\hline 9 & Ac & $\mathrm{CO}_{2} \mathrm{Me}$ & $\mathrm{CsOAc}(0.5)$ & DMF & NR \\
\hline 10 & $\mathrm{Ac}$ & $\mathrm{CO}_{2} \mathrm{Me}$ & $\mathrm{NaOAc}(0.5)$ & $\mathrm{MeOH}$ & 53 \\
\hline 11 & $\mathrm{Ac}$ & $\mathrm{CO}_{2} \mathrm{Me}$ & $\mathrm{K}_{2} \mathrm{CO}_{3}(0.5)$ & $\mathrm{MeOH}$ & 47 \\
\hline 12 & $\mathrm{Ac}$ & $\mathrm{CO}_{2} \mathrm{Me}$ & $\mathrm{DBU}(0.5)$ & $\mathrm{MeOH}$ & 34 \\
\hline 13 & Piv & $\mathrm{CO}_{2} \mathrm{Me}$ & $\mathrm{CsOAc}(0.5)$ & $\mathrm{MeOH}$ & 19 \\
\hline 14 & Ts & $\mathrm{CO}_{2} \mathrm{Me}$ & $\mathrm{CsOAc}(0.5)$ & $\mathrm{MeOH}$ & 40 \\
\hline 15 & $\mathrm{Ac}$ & Ac & $\mathrm{CsOAc}(0.5)$ & $\mathrm{MeOH}$ & 25 \\
\hline 16 & $\mathrm{Ac}$ & Ts & $\mathrm{CsOAc}(0.5)$ & $\mathrm{MeOH}$ & 18 \\
\hline 17 & $\mathrm{Ac}$ & Boc & CsOAc (0.5), HOAc (1) & $\mathrm{MeOH}$ & 48 \\
\hline 18 & Ac & Boc & $\mathrm{CsOAc}(0.5), \mathrm{K}_{2} \mathrm{CO}_{3}(0.5)$ & $\mathrm{MeOH}$ & 90 \\
\hline 19 & Ac & Boc & 1 & $\mathrm{MeOH}$ & NR \\
\hline $20^{c}$ & $\mathrm{Ac}$ & Boc & $\mathrm{CsOAc}(0.5), \mathrm{K}_{2} \mathrm{CO}_{3}(0.5)$ & $\mathrm{MeOH}$ & NR \\
\hline
\end{tabular}

${ }^{a}$ Reaction conditions: $1(0.1 \mathrm{mmol}), 2(0.12 \mathrm{mmol}),\left[\mathrm{Cp}^{*} \mathrm{RhCl}_{2}\right]_{2}(2.5 \mathrm{~mol} \%)$ and additives in solvent $(0.2 \mathrm{M})$ at $\mathrm{rt}$ for $24 \mathrm{~h}$ under air. ${ }^{b 1} \mathrm{H}$ NMR yield. ${ }^{c} \mathrm{No}\left[\mathrm{Cp} * \mathrm{RhCl}_{2}\right]_{2}$ was used.

\section{General Procedure:}

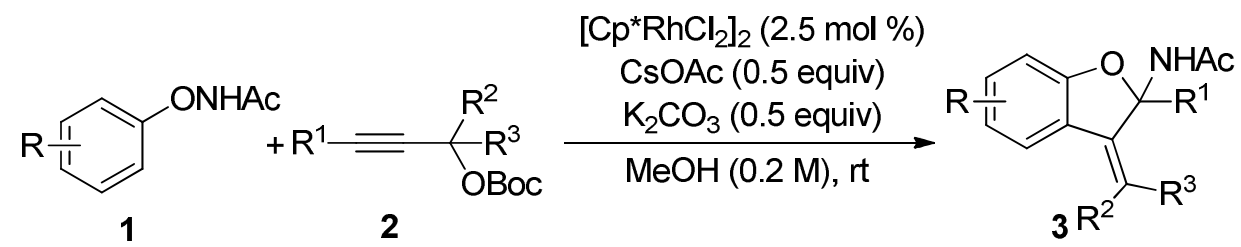

Without any particular precautions to extrude oxygen or moisture, the aryloxyacetamide 1 ( 1 equiv), the tert-butyl propargyl carbonate $\mathbf{2}$ (1.2 equiv), [Cp* $\left.\mathrm{RhCl}_{2}\right]_{2}(2.5 \mathrm{~mol} \%), \mathrm{CsOAc}\left(0.5\right.$ equiv) and $\mathrm{K}_{2} \mathrm{CO}_{3}(0.5$ equiv) were weighted in a $4 \mathrm{~mL}$ vial equipped with a stir bar. $\mathrm{MeOH}(0.2 \mathrm{M})$ was then added. The reaction was stirred at room temperature and monitored by TLC. Afterwards, it was diluted with EtOAc and transferred to a round bottom flask. Silica gel was added to the flask 
and volatiles were evaporated under reduced pressure. The purification was performed by flash column chromatography on silica gel (see below for specific eluents).

\section{Characterization of products 3:}<smiles>C=C1c2ccccc2OC1(NC(C)=O)c1ccccc1</smiles>

Yield 88\% (47 mg); white solid; m.p. 170-171 ${ }^{\circ} \mathrm{C} ;{ }^{1}$ H NMR (400 MHz, DMSO-d 6 ): $\delta 8.99(\mathrm{~s}, 1 \mathrm{H}), 7.50-7.44(\mathrm{~m}, 3 \mathrm{H}), 7.39-7.26(\mathrm{~m}, 4 \mathrm{H}), 6.98-6.92(\mathrm{~m}, 2 \mathrm{H}), 5.59(\mathrm{~s}, 1 \mathrm{H})$, 5.14 (s, 1H), 1.91 (s, 3H); ${ }^{13}$ C NMR (100 MHz, CDCl 3 ): $\delta 169.9,160.6,148.4,140.7$, 131.1, 128.8, 128.5, 124.6, 123.9, 121.5, 110.3, 104.5, 96.4, 23.7; IR (neat, $\mathbf{c m}^{-1}$ ): v 3283, 2922, 1674, 1525, 1244, 941, 744, 696; MS (m/z, ESI): 266, 207; HRMS calculated for $\mathrm{C}_{17} \mathrm{H}_{16} \mathrm{NO}_{2}(\mathrm{M}+\mathrm{H})^{+}: 266.1176$; Found: $266.1165 ; \mathbf{R}_{\mathbf{f}}=0.5$ (PE: EtOAc, 1:1).

X-ray crystallography of $\mathbf{3 a a}$ :
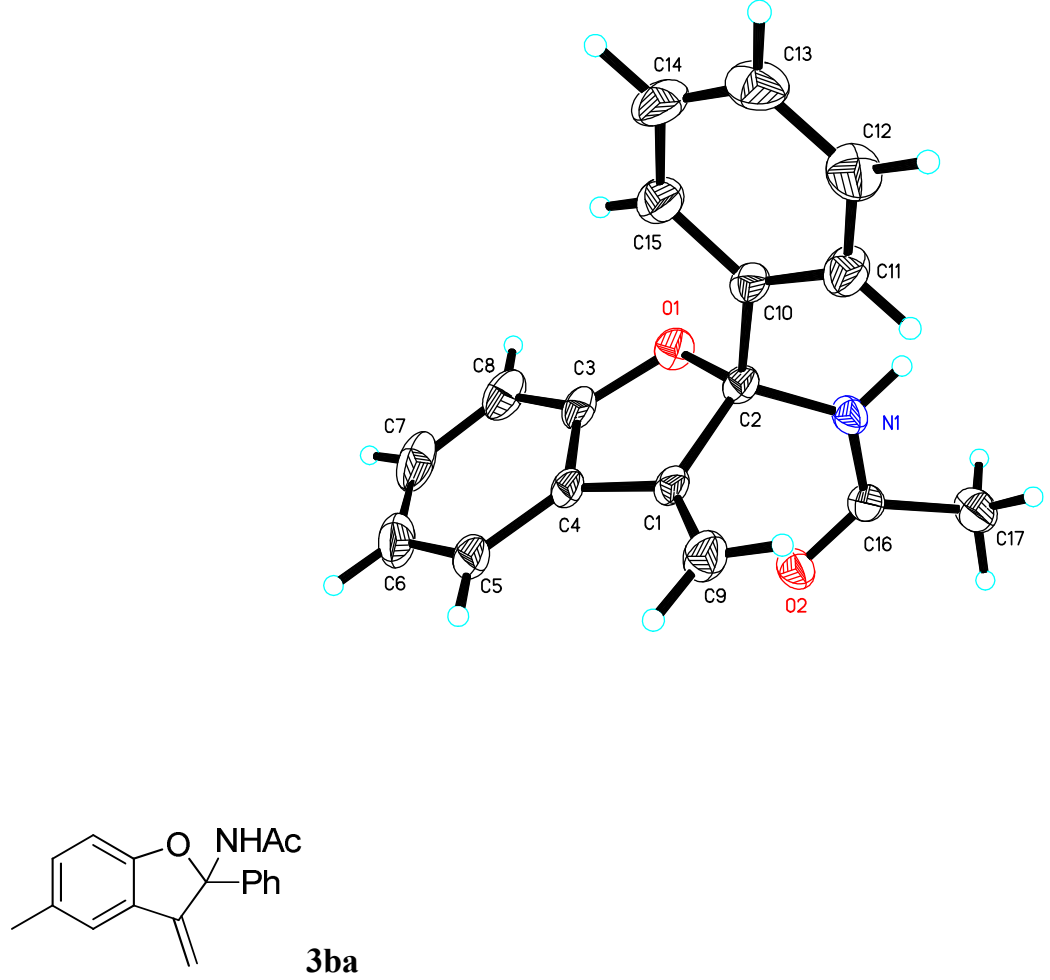
Yield 81\% (45 mg); white solid; m.p. 173-174 ${ }^{\circ} \mathrm{C} ;{ }^{1}$ H NMR (400 MHz, DMSO-d 6 ): $\delta 8.96(\mathrm{~s}, 1 \mathrm{H}), 7.44$ (d, $J=7.2 \mathrm{~Hz}, 2 \mathrm{H}), 7.38-7.29$ (m, 4H), 7.08 (d, $J=8.0 \mathrm{~Hz}, 1 \mathrm{H})$, $6.85(\mathrm{~d}, J=8.0 \mathrm{~Hz}, 1 \mathrm{H}), 5.53(\mathrm{~s}, 1 \mathrm{H}), 5.10(\mathrm{~s}, 1 \mathrm{H}), 2.26(\mathrm{~s}, 3 \mathrm{H}), 1.91(\mathrm{~s}, 3 \mathrm{H}) ;{ }^{13} \mathrm{C}$ NMR (100 MHz, DMSO-d $)$ ): $\delta$ 169.6, 158.2, 147.8, 141.5, 131.2, 129.8, 128.4, 127.9, 124.6, 124.2, 121.8, 109.2, 104.0, 96.3, 23.3, 20.4; IR (neat, $\mathbf{c m}^{-1}$ ): v 3247, 2922, 1671, 1483, 1248, 954, 698; MS (m/z, ESI): 280, 221; HRMS calculated for $\mathrm{C}_{18} \mathrm{H}_{18} \mathrm{NO}_{2}(\mathrm{M}+\mathrm{H})^{+}: 280.1332 ;$ Found: 280.1327; $\mathbf{R}_{\mathbf{f}}=0.4$ (PE: EtOAc, 1:1).

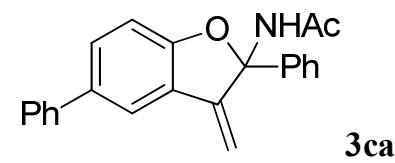

Yield 84\% (57 mg); light yellow solid; m.p. 185-186 ${ }^{\circ} \mathrm{C} ;{ }^{1} \mathbf{H}$ NMR (400 MHz, DMSO-d $\left.)_{6}\right): \delta 9.06(\mathrm{~s}, 1 \mathrm{H}), 7.82(\mathrm{~d}, J=2.0 \mathrm{~Hz}, 1 \mathrm{H}), 7.64(\mathrm{~d}, J=7.6 \mathrm{~Hz}, 2 \mathrm{H}), 7.58(\mathrm{dd}$, $\left.J_{1}=8.4 \mathrm{~Hz}, J_{2}=2.0 \mathrm{~Hz}, 1 \mathrm{H}\right), 7.51-7.30(\mathrm{~m}, 8 \mathrm{H}), 7.06(\mathrm{~d}, J=8.4 \mathrm{~Hz}, 1 \mathrm{H}), 5.75(\mathrm{~s}$, 1H), 5.19 (s, 1H), 1.93 (s, 3H); ${ }^{13}$ C NMR (100 MHz, DMSO-d 6 ): $\delta$ 169.7, 159.8, $147.3,141.3,140.1,133.6,129.4,128.9,128.5,128.1,126.8,126.4,125.2,124.7$, 120.0, 110.0, 105.0, 96.9, 23.3; IR (neat, $\mathbf{c m}^{-1}$ ): $v$ 3194, 2921, 1667, 1537, 1471, 1250, 946, 696; MS (m/z, ESI): 342, 283; HRMS calculated for $\mathrm{C}_{23} \mathrm{H}_{20} \mathrm{NO}_{2}(\mathrm{M}+\mathrm{H})^{+}$: 342.1489; Found: 342.1478; $\mathbf{R}_{\mathbf{f}}=0.4$ (PE: EtOAc, 1:1).

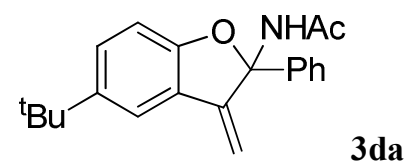

Yield 84\% (54 mg); white solid; m.p. 183-185 ${ }^{\circ} \mathrm{C} ;{ }^{1}$ H NMR (400 MHz, DMSO-d 6 ): $\delta 8.95(\mathrm{~s}, 1 \mathrm{H}), 7.49\left(\mathrm{dd}, J_{1}=7.2 \mathrm{~Hz}, J_{2}=2.0 \mathrm{~Hz}, 2 \mathrm{H}\right), 7.45(\mathrm{~s}, 1 \mathrm{H}), 7.38-7.28(\mathrm{~m}, 4 \mathrm{H})$, $6.89(\mathrm{~d}, J=8.8 \mathrm{~Hz}, 1 \mathrm{H}), 5.62(\mathrm{~s}, 1 \mathrm{H}), 5.11(\mathrm{~s}, 1 \mathrm{H}), 1.91(\mathrm{~s}, 3 \mathrm{H}), 1.28(\mathrm{~s}, 9 \mathrm{H}) ;{ }^{13} \mathrm{C}$ NMR (100 MHz, DMSO-d $\left.{ }_{6}\right): \delta 169.6,158.1,147.9,143.4,141.5,128.4,127.9$, 127.7, 124.7, 123.8, 118.3, 108.9, 104.0, 96.5, 34.2, 31.5, 23.3; IR (neat, $\mathbf{c m}^{-1}$ ): v 3259, 3211, 2953, 1675, 1547, 1485, 1248, 947, 697; MS (m/z, ESI): 322, 263; HRMS calculated for $\mathrm{C}_{21} \mathrm{H}_{24} \mathrm{NO}_{2}(\mathrm{M}+\mathrm{H})^{+}: 322.1802$; Found: $322.1791 ; \mathbf{R}_{\mathbf{f}}=0.5$ (PE: 
EtOAc, 1:1).

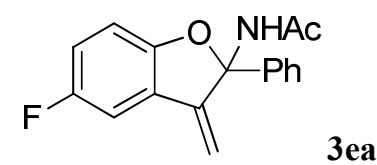

Yield 67\% (38 mg); brown solid; m.p. $157-158{ }^{\circ} \mathrm{C} ;{ }^{1} \mathbf{H}$ NMR (400 MHz, DMSO-d 6 ): $\delta 9.06(\mathrm{~s}, 1 \mathrm{H}), 7.47(\mathrm{~d}, J=7.2 \mathrm{~Hz}, 2 \mathrm{H}), 7.43-7.31(\mathrm{~m}, 4 \mathrm{H}), 7.10\left(\mathrm{td}, J_{1}=8.8 \mathrm{~Hz}, J_{2}=\right.$ $2.4 \mathrm{~Hz}, 1 \mathrm{H}), 6.97\left(\mathrm{dd}, J_{l}=8.4 \mathrm{~Hz}, J_{2}=4.0 \mathrm{~Hz}, 1 \mathrm{H}\right), 5.68(\mathrm{~s}, 1 \mathrm{H}), 5.21(\mathrm{~s}, 1 \mathrm{H}), 1.92(\mathrm{~s}$, $3 \mathrm{H}) ;{ }^{13}$ C NMR (100 MHz, DMSO-d $\left.{ }_{6}\right): \delta 169.7,157.2\left(\mathrm{~d}, J_{C-F}=233.8 \mathrm{~Hz}\right), 156.2$, $147.1\left(\mathrm{~d}, J_{C-F}=3.4 \mathrm{~Hz}\right), 141.1,128.5,128.2,125.8\left(\mathrm{~d}, J_{C-F}=9.3 \mathrm{~Hz}\right), 124.7,116.9(\mathrm{~d}$, $\left.J_{C-F}=24.5 \mathrm{~Hz}\right), 110.3\left(\mathrm{~d}, J_{C-F}=8.2 \mathrm{~Hz}\right), 108.3\left(\mathrm{~d}, J_{C-F}=25.3 \mathrm{~Hz}\right), 106.1,97.0,23.2$; ${ }^{19}$ F NMR (376 MHz, DMSO-d 6 ): -122.8; IR (neat, cm$^{-1}$ ): v 3258, 3024, 1674, 1531, 1473, 1194, 948, 808, 693; MS (m/z, ESI): 284, 225; HRMS calculated for $\mathrm{C}_{17} \mathrm{H}_{15} \mathrm{FNO}_{2}(\mathrm{M}+\mathrm{H})^{+}:$284.1081; Found: 284.1070; $\mathbf{R}_{\mathbf{f}}=0.3$ (PE: EtOAc, 1:1).

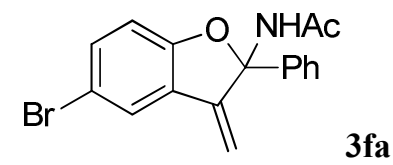

Yield 74\% (51 mg); white solid; m.p. 176-178 ${ }^{\circ} \mathrm{C} ;{ }^{1}$ H NMR (400 MHz, DMSO-d 6 ): $\delta 9.11(\mathrm{~s}, 1 \mathrm{H}), 7.76(\mathrm{~d}, J=2.0 \mathrm{~Hz}, 1 \mathrm{H}), 7.48-7.32(\mathrm{~m}, 6 \mathrm{H}), 6.95(\mathrm{~d}, J=8.8 \mathrm{~Hz}, 1 \mathrm{H})$, $5.73(\mathrm{~s}, 1 \mathrm{H}), 5.21(\mathrm{~s}, 1 \mathrm{H}), 1.92(\mathrm{~s}, 3 \mathrm{H}) ;{ }^{13} \mathbf{C}$ NMR (100 MHz, DMSO-d 6 ): $\delta$ 169.8, $159.1,146.2,140.8,132.9,128.6,128.3,127.2,124.7,124.4,112.4,111.7,106.3$, 97.1, 23.2; IR (neat, $\mathbf{c m}^{-1}$ ): v 3244, 3028, 1668, 1531, 1461, 1247, 950, 812; MS (m/z, ESI): 344, 285; HRMS calculated for $\mathrm{C}_{17} \mathrm{H}_{15} \mathrm{BrNO}_{2}(\mathrm{M}+\mathrm{H})^{+}$: 344.0281 ; Found: $344.0279 ; \mathbf{R}_{\mathbf{f}}=0.6(\mathrm{DCM}:$ EtOAc, $10: 1)$.

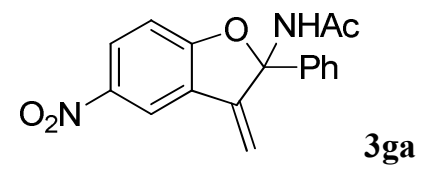

Yield 37\% (23 mg); orange solid; m.p. $157-159{ }^{\circ} \mathrm{C} ;{ }^{1} \mathbf{H}$ NMR (400 MHz, $\left.\mathbf{C D C l}_{3}\right): \delta$ $8.30(\mathrm{~d}, J=2.4 \mathrm{~Hz}, 1 \mathrm{H}), 8.22(\mathrm{~d}, J=8.4 \mathrm{~Hz}, 1 \mathrm{H}), 7.54-7.52(\mathrm{~m}, 2 \mathrm{H}), 7.45-7.38(\mathrm{~m}$, 
3H), $7.01(\mathrm{~d}, J=8.8 \mathrm{~Hz}, 1 \mathrm{H}), 6.64(\mathrm{~s}, 1 \mathrm{H}), 5.79(\mathrm{~s}, 1 \mathrm{H}), 5.36(\mathrm{~s}, 1 \mathrm{H}), 2.05(\mathrm{~s}, 3 \mathrm{H})$;

${ }^{13}$ C NMR (100 MHz, DMSO-d $)$ ): $\delta$ 170.1, 164.5, 144.8, 141.9, 140.0, 128.8, 127.1, 126.3, 124.8, 117.9, 110.0, 108.3, 99.0, 23.2; IR (neat, $\mathbf{c m}^{-1}$ ): $v 3284,2926,2855$, 1680, 1519, 1337, 1261, 941, 898; MS (m/z, ESI): 328, 252; HRMS calculated for $\mathrm{C}_{17} \mathrm{H}_{18} \mathrm{~N}_{3} \mathrm{O}_{4}\left(\mathrm{M}+\mathrm{NH}_{4}\right)^{+}:$328.1292; Found: 328.1293; $\mathbf{R}_{\mathbf{f}}=0.3$ (PE: EtOAc, 1:1).

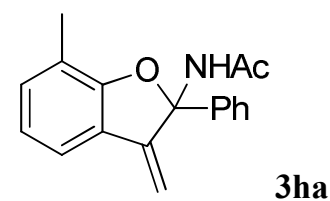

Yield 90\% (50 mg); light yellow solid; m.p. 166-167 ${ }^{\circ} \mathrm{C} ;{ }^{1} \mathbf{H}$ NMR (400 MHz,

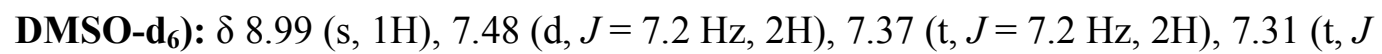
$=7.2 \mathrm{~Hz}, 2 \mathrm{H}), 7.12(\mathrm{~d}, J=7.2 \mathrm{~Hz}, 1 \mathrm{H}), 6.85(\mathrm{t}, J=7.6 \mathrm{~Hz}, 1 \mathrm{H}), 5.54(\mathrm{~s}, 1 \mathrm{H}), 5.15(\mathrm{~s}$, 1H), 2.26 (s, 3H), 1.93 (s, 3H); ${ }^{13}$ C NMR (100 MHz, DMSO-d 6 ): $\delta$ 169.5, 158.5, $148.1,141.5,131.5,128.4,128.0,124.6,123.7,120.9,119.1,104.0,96.1,23.3,14.9$;

IR (neat, $\left.\mathbf{c m}^{-1}\right):$ v 3253, 3030, 1693, 1539, 966, 744, 694; MS (m/z, ESI): 280, 221; HRMS calculated for $\mathrm{C}_{18} \mathrm{H}_{18} \mathrm{NO}_{2}(\mathrm{M}+\mathrm{H})^{+}:$: 280.1332; Found: 280.1332; $\mathbf{R}_{\mathbf{f}}=0.5$ (PE: EtOAc, 1:1).

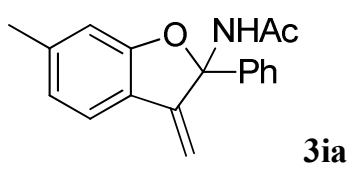

Yield 79\% (44 mg); white solid; m.p. $158-160{ }^{\circ} \mathrm{C} ;{ }^{1}$ H NMR (400 MHz, DMSO-d 6 ): $\delta 8.96(\mathrm{~s}, 1 \mathrm{H}), 7.46(\mathrm{~d}, J=7.2 \mathrm{~Hz}, 2 \mathrm{H}), 7.39-7.28(\mathrm{~m}, 4 \mathrm{H}), 7.11(\mathrm{~d}, J=7.2 \mathrm{~Hz}, 1 \mathrm{H})$, $6.84(\mathrm{t}, J=7.6 \mathrm{~Hz}, 1 \mathrm{H}), 5.53(\mathrm{~s}, 1 \mathrm{H}), 5.14(\mathrm{~s}, 1 \mathrm{H}), 2.25(\mathrm{~s}, 3 \mathrm{H}), 1.92(\mathrm{~s}, 3 \mathrm{H}) ;{ }^{13} \mathrm{C}$ NMR (100 MHz, DMSO-d $\left.{ }_{6}\right): \delta$ 169.6, 158.5, 148.1, 141.5, 131.5, 128.5, 128.0, 124.7, 123.7, 120.9, 119.1, 104.1, 96.1, 23.3, 14.9; IR (neat, $\mathbf{c m}^{-1}$ ): v 3256, 3035, 1671, 1536, 967, 747, 695; MS (m/z, ESI): 280, 221; HRMS calculated for $\mathrm{C}_{18} \mathrm{H}_{18} \mathrm{NO}_{2}(\mathrm{M}+\mathrm{H})^{+}:$280.1332; Found: 280.1326; $\mathbf{R}_{\mathbf{f}}=0.5$ (DCM: EtOAc, 10:1). 


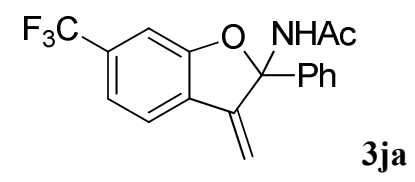

Yield 50\% (33 mg); white solid; m.p. 179-180 ${ }^{\circ} \mathrm{C} ;{ }^{1} \mathbf{H}$ NMR (400 MHz, DMSO-d $\mathbf{d}_{6}$ ): $\delta 9.20(\mathrm{~s}, 1 \mathrm{H}), 7.72(\mathrm{~d}, J=7.6 \mathrm{~Hz}, 1 \mathrm{H}), 7.51(\mathrm{~d}, J=7.2 \mathrm{~Hz}, 2 \mathrm{H}), 7.43-7.28$ (m, 5H), 5.81 (s, 1H), 5.33 (s, 1H), 1.92 (s, 3H); ${ }^{13}$ C NMR (100 MHz, DMSO-d 6 ): $\delta$ 170.0, 159.9, 146.0, 140.6, 128.9, 128.7, 128.5, 124.8, 122.8, 122.4, 117.9, 107.6, 106.5, 97.3, 23.2; ${ }^{19}$ F NMR (376 MHz, DMSO-d ( $_{\text {): }}$-60.8; IR (neat, $\mathbf{~ c m}^{-1}$ ): v 3259, 2924, 1671, 1537, 1437, 1328, 1154, 1121，948; MS (m/z， ESI): 334, 275; HRMS calculated for $\mathrm{C}_{18} \mathrm{H}_{15} \mathrm{~F}_{3} \mathrm{NO}_{2}(\mathrm{M}+\mathrm{H})^{+}$: 334.1049; Found: 334.1046; $\mathbf{R}_{\mathbf{f}}=0.5$ (PE: EtOAc, 1:1).

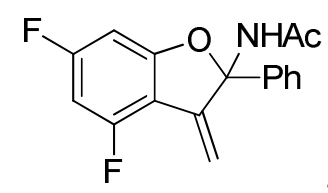

Yield 63\% (38 mg); white solid; m.p. $174-176{ }^{\circ} \mathrm{C} ;{ }^{1} \mathbf{H}$ NMR (400 MHz, DMSO-d 6 ): $\delta 9.22(\mathrm{~s}, 1 \mathrm{H}), 7.49$ (d, $J=6.8 \mathrm{~Hz}, 2 \mathrm{H}), 7.43-7.37$ (m, 3H), 6.86-6.82 (m, 2H), 5.52 (s, 1H), 5.30 (s, 1H), 1.93 (s, 3H); ${ }^{13}$ C NMR (100 MHz, DMSO-d d $_{\text {): }} \delta$ 169.8, 164.8 (dd, $\left.J_{C-F}=247.7,11.0 \mathrm{~Hz}\right), 162.2\left(\mathrm{t}, J_{C-F}=13.0 \mathrm{~Hz}\right), 159.1\left(\mathrm{dd}, J_{C-F}=252.8,14.9 \mathrm{~Hz}\right)$, 143.5, 140.0, 129.1, 124.6, $109.0\left(\mathrm{~d}, J_{C-F}=17.9 \mathrm{~Hz}\right), 108.5,98.2,97.4\left(\mathrm{t}, J_{C-F}=25.6\right.$ $\mathrm{Hz}), 94.8\left(\mathrm{~d}, J_{C-F}=26.0 \mathrm{~Hz}\right), 23.9$; ${ }^{19}$ F NMR (376 MHz, DMSO-d $\left.)_{6}\right):-107.4,-116.2$; IR (neat, $\left.\mathbf{c m}^{-1}\right):$ v 3305, 1681, 1493, 1118, 989, 912, 833; MS (m/z, ESI): 302, 243, 221; HRMS calculated for $\mathrm{C}_{17} \mathrm{H}_{14} \mathrm{~F}_{2} \mathrm{NO}_{2}(\mathrm{M}+\mathrm{H})^{+}:$302.0987; Found: 302.0980; $\mathbf{R}_{\mathbf{f}}=$ 0.7 (DCM: EtOAc, 10:1).

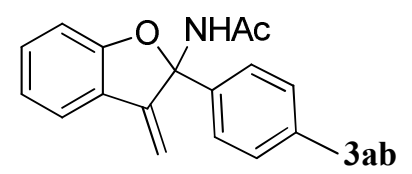

Yield 81\% (45 mg); brown solid; m.p. 170-171 ${ }^{\circ} \mathrm{C} ;{ }^{1} \mathbf{H}$ NMR (400 MHz, DMSO-d 6 ): $\delta 8.96$ (s, 1H), 7.49 (d, $J=7.6 \mathrm{~Hz}, 1 \mathrm{H}), 7.35$ (d, $J=8.4 \mathrm{~Hz}, 2 \mathrm{H}), 7.28$ (t, $J=8.4 \mathrm{~Hz}$, $1 \mathrm{H}), 7.17(\mathrm{~d}, J=8.4 \mathrm{~Hz}, 2 \mathrm{H}), 6.97-6.91(\mathrm{~m}, 2 \mathrm{H}), 5.58(\mathrm{~s}, 1 \mathrm{H}), 5.11(\mathrm{~s}, 1 \mathrm{H}), 2.28(\mathrm{~s}$, 
3H), 1.91 (s, 3H); ${ }^{13}$ C NMR (100 MHz, DMSO-d 6 ): $\delta$ 169.5, 160.1, 147.6, 138.5, $137.3,130.6,128.9,124.7,124.5,121.6,120.9,109.6,104.1,96.3,23.3,20.6$; IR (neat, $\left.\mathbf{c m}^{-1}\right): ~ v 3295,1675,1509,1464,1245,864,751 ;$ MS (m/z, ESI): 280, 221; HRMS calculated for $\mathrm{C}_{18} \mathrm{H}_{18} \mathrm{NO}_{2}(\mathrm{M}+\mathrm{H})^{+}:$280.1332; Found: 280.1332; $\mathbf{R}_{\mathbf{f}}=0.6(\mathrm{PE}$ : EtOAc, 1:1).

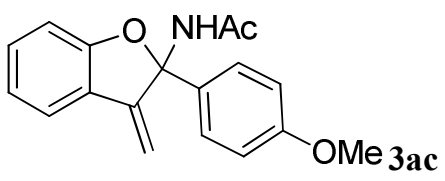

Yield 71\% (42 mg); white solid; m.p. 149-150 ${ }^{\circ} \mathrm{C} ;{ }^{1}$ H NMR (400 MHz, DMSO-d 6 ): $\delta 8.95(\mathrm{~s}, 1 \mathrm{H}), 7.49(\mathrm{~d}, J=7.6 \mathrm{~Hz}, 1 \mathrm{H}), 7.39\left(\mathrm{dd}, J_{1}=6.8 \mathrm{~Hz}, J_{2}=2.0 \mathrm{~Hz}, 2 \mathrm{H}\right), 7.27(\mathrm{t}$, $J=8.0 \mathrm{~Hz}, 1 \mathrm{H}), 6.95-6.91(\mathrm{~m}, 4 \mathrm{H}), 5.75(\mathrm{~s}, 1 \mathrm{H}), 5.10(\mathrm{~s}, 1 \mathrm{H}), 3.73(\mathrm{~s}, 3 \mathrm{H}), 1.91(\mathrm{~s}$, $3 \mathrm{H}) ;{ }^{13}$ C NMR (100 MHz, DMSO-d 6 ): $\delta$ 169.6, 160.0, 159.1, 147.7, 133.3, 130.6, 126.2, 124.6, 121.7, 120.8, 113.7, 109.6, 103.9, 96.3, 55.2, 23.3; IR (neat, $\mathbf{c m}^{-1}$ ): v 3289, 1674, 1463, 1369, 1248, 1170, 866, 751; MS (m/z, ESI): 296, 237; HRMS calculated for $\mathrm{C}_{18} \mathrm{H}_{18} \mathrm{NO}_{3}(\mathrm{M}+\mathrm{H})^{+}:$296.1281; Found: $296.1271 ; \mathbf{R}_{\mathbf{f}}=0.4(\mathrm{DCM}$ : EtOAc, 10:1).

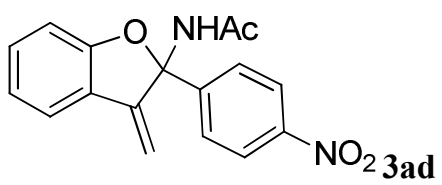

Yield 94\% (58 mg); orange solid; m.p. 179-180 ${ }^{\circ}$ C; ${ }^{1}$ H NMR (400 MHz, DMSO-d 6 ): $\delta 9.20(\mathrm{~s}, 1 \mathrm{H}), 8.21(\mathrm{~d}, J=8.8 \mathrm{~Hz}, 2 \mathrm{H}), 7.67(\mathrm{~d}, J=8.8 \mathrm{~Hz}, 2 \mathrm{H}), 7.55(\mathrm{~d}, J=7.6 \mathrm{~Hz}$, 1H), 7.35 (t, $J=7.6 \mathrm{~Hz}, 1 \mathrm{H}), 7.07(\mathrm{~d}, J=7.6 \mathrm{~Hz}, 1 \mathrm{H}), 7.00(\mathrm{t}, J=7.6 \mathrm{~Hz}, 1 \mathrm{H}), 5.74(\mathrm{~s}$, 1H), 5.25 (s, 1H), 1.95 (s, 3H); ${ }^{13}$ C NMR (100 MHz, DMSO-d 6 ): $\delta$ 170.0, 159.9, $148.7,147.0,146.9,131.3,125.9,123.9,123.4,122.0,121.7,110.2,106.6,95.5,23.2$; IR (neat, $\mathbf{c m}^{-1}$ ): $v$ 3268, 1672, 1513, 1346, 1275, 959, 850, 734; MS (m/z, ESI): 311, 252; HRMS calculated for $\mathrm{C}_{17} \mathrm{H}_{15} \mathrm{~N}_{2} \mathrm{O}_{4}(\mathrm{M}+\mathrm{H})^{+}: 311.1026$; Found: $311.1026 ; \mathbf{R}_{\mathbf{f}}=0.7$ (DCM: EtOAc, 5:1). 


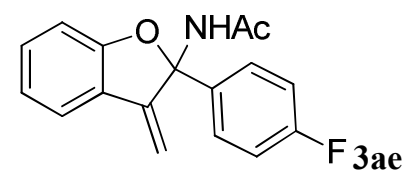

Yield 83\% (47 mg); white solid; m.p. 173-174 ${ }^{\circ} \mathrm{C} ;{ }^{1}$ H NMR (400 MHz, DMSO-d 6 ): $\delta 9.02(\mathrm{~s}, 1 \mathrm{H}), 7.52-7.46(\mathrm{~m}, 3 \mathrm{H}), 7.29\left(\mathrm{td}, J_{1}=8.0 \mathrm{~Hz}, J_{2}=0.8 \mathrm{~Hz}, 1 \mathrm{H}\right), 7.55\left(\mathrm{td}, J_{1}\right.$ $\left.=8.8 \mathrm{~Hz}, J_{2}=2.0 \mathrm{~Hz}, 2 \mathrm{H}\right), 6.99-6.93(\mathrm{~m}, 2 \mathrm{H}), 5.62(\mathrm{~s}, 1 \mathrm{H}), 5.16(\mathrm{~s}, 1 \mathrm{H}), 1.91(\mathrm{~s}, 3 \mathrm{H})$; ${ }^{13}$ C NMR (100 MHz, DMSO-d 6 ): $\delta 169.7,161.9$ (d, $\left.J_{C-F}=242.9 \mathrm{~Hz}\right), 159.9,147.5$, $137.6\left(\mathrm{~d}, J_{C-F}=3.1 \mathrm{~Hz}\right), 130.8,127.0\left(\mathrm{~d}, J_{C-F}=8.4 \mathrm{~Hz}\right), 124.2,121.8,121.2,115.2(\mathrm{~d}$, $J_{C-F}=21.3 \mathrm{~Hz}$ ), 109.8, 104.8, 95.9, 23.3; ${ }^{19}$ F NMR (376 MHz, DMSO-d 6 ): -114.9; IR (neat, $\left.\mathbf{c m}^{-1}\right):$ v 3262, 1671, 1534, 1503, 1465, 1247, 1224, 830, 747; MS (m/z, ESI): 284, 225; HRMS calculated for $\mathrm{C}_{17} \mathrm{H}_{15} \mathrm{FNO}_{2}(\mathrm{M}+\mathrm{H})^{+}$: 284.1081; Found: 284.1076; $\mathbf{R}_{\mathbf{f}}=0.5(\mathrm{PE}:$ EtOAc, $1: 1)$.

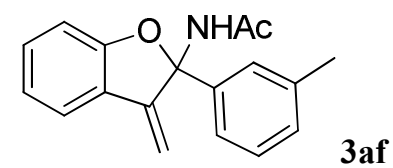

Yield 79\% (44 mg); white solid; m.p. $150-151{ }^{\circ} \mathrm{C} ;{ }^{1} \mathbf{H}$ NMR (400 MHz, DMSO-d 6 ): $\delta 8.99(\mathrm{~s}, 1 \mathrm{H}), 7.49(\mathrm{~d}, J=7.2 \mathrm{~Hz}, 2 \mathrm{H}), 7.30-7.24(\mathrm{~m}, 4 \mathrm{H}), 7.14-7.12(\mathrm{~m}, 1 \mathrm{H})$, 6.98-6.91 (m, 2H), 5.58 (s, 1H), $5.13(\mathrm{~s}, 1 \mathrm{H}), 2.30$ (s, 3H), $1.91(\mathrm{~s}, 3 \mathrm{H}) ;{ }^{13} \mathbf{C}$ NMR (100 MHz, DMSO-d 6 ): $\delta 169.7,160.1,147.5,141.3,137.7,130.7,128.7,128.4$, 125.2, 124.5, 121.9, 121.7, 121.0, 109.7, 104.4, 96.3, 23.3, 21.3; IR (neat, $\mathbf{c m}^{-1}$ ): v 3307, 1679, 1519, 1244, 955, 743; MS (m/z, ESI): 280, 221; HRMS calculated for $\mathrm{C}_{18} \mathrm{H}_{18} \mathrm{NO}_{2}(\mathrm{M}+\mathrm{H})^{+}: 280.1332 ;$ Found: 280.1326; $\mathbf{R}_{\mathbf{f}}=0.5$ (PE: EtOAc, 1:1).

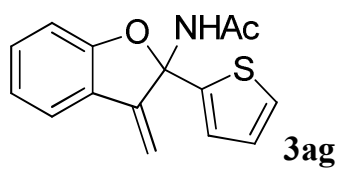

Yield 85\% (46 mg); white solid; m.p. 150-152 ${ }^{\circ} \mathrm{C} ;{ }^{1}$ H NMR (400 MHz, DMSO-d 6 ): $\delta 9.18(\mathrm{~s}, 1 \mathrm{H}), 7.54-7.49(\mathrm{~m}, 2 \mathrm{H}), 7.28-7.21(\mathrm{~m}, 2 \mathrm{H}), 7.03(\mathrm{t}, J=4.4 \mathrm{~Hz}, 1 \mathrm{H})$, 6.96-6.88 (m, 2H), 5.67 (s, 1H), 5.23 (s, 1H), 1.90 (s, 3H); ${ }^{13}$ C NMR (100 MHz, DMSO-d $\left.{ }_{6}\right): \delta 169.4,159.5,147.2,145.1,130.6,127.2,126.3,124.6,124.4,121.7$, 
121.0, 109.7, 104.1, 94.7, 23.2; IR (neat, $\mathbf{c m}^{-1}$ ): v 3271, 1673, 1520, 1202, 881, 710;

MS (m/z, ESI): 272, 213; HRMS calculated for $\mathrm{C}_{15} \mathrm{H}_{14} \mathrm{NO}_{2} \mathrm{~S}(\mathrm{M}+\mathrm{H})^{+}:$: 272.0740;

Found: $272.0741 ; \mathbf{R}_{\mathbf{f}}=0.6(\mathrm{DCM}$ : EtOAc, 10:1).

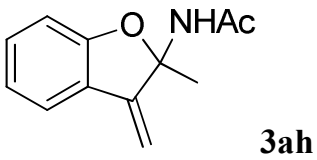

Yield 42\% (17 mg); white solid; m.p. $112-114{ }^{\circ} \mathrm{C} ;{ }^{1}$ H NMR (400 MHz, DMSO-d ${ }_{6}$ ): $\delta$ $8.84(\mathrm{~s}, 1 \mathrm{H}), 7.48\left(\mathrm{dd}, J_{1}=7.6 \mathrm{~Hz}, J_{2}=0.8 \mathrm{~Hz}, 1 \mathrm{H}\right), 7.20(\mathrm{~m}, 1 \mathrm{H}), 6.88\left(\mathrm{td}, J_{1}=7.6\right.$ $\left.\mathrm{Hz}, J_{2}=0.8 \mathrm{~Hz}, 1 \mathrm{H}\right), 6.75(\mathrm{~d}, J=8.4 \mathrm{~Hz}, 1 \mathrm{H}), 5.46(\mathrm{~s}, 1 \mathrm{H}), 4.95(\mathrm{~s}, 1 \mathrm{H}), 1.81(\mathrm{~s}, 3 \mathrm{H})$, $1.52(\mathrm{~s}, 3 \mathrm{H}) ;{ }^{13} \mathbf{C}$ NMR (100 MHz, DMSO-d 6 ): $\delta 168.7,159.5,148.5,130.3,125.0$, 121.3, 120.2, 109.6, 101.4, 94.4, 27.5, 23.1; IR (neat, $\mathbf{c m}^{-1}$ ): v 3293, 2925, 1676, 1547, 1465, 1259, 878, 750; MS (m/z, ESI): 204, 145; HRMS calculated for $\mathrm{C}_{12} \mathrm{H}_{14} \mathrm{NO}_{2}(\mathrm{M}+\mathrm{H})^{+}:$204.1019; Found: 204.1021; $\mathbf{R}_{\mathbf{f}}=0.4$ (DCM: EtOAc, 10:1).

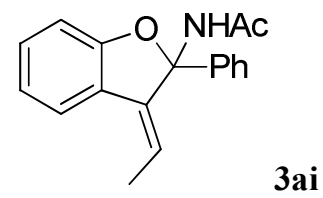

Yield 81\% (45 mg); white solid; m.p. $185-187{ }^{\circ} \mathrm{C} ;{ }^{1}$ H NMR (400 MHz, DMSO-d 6 ): $\delta 8.88(\mathrm{~s}, 1 \mathrm{H}), 7.55(\mathrm{~d}, J=7.6 \mathrm{~Hz}, 1 \mathrm{H}), 7.41-7.24(\mathrm{~m}, 6 \mathrm{H}), 6.99-6.94(\mathrm{~m}, 2 \mathrm{H}), 5.66(\mathrm{q}$, $J=7.2 \mathrm{~Hz}, 1 \mathrm{H}), 1.93(\mathrm{~d}, J=7.6 \mathrm{~Hz}, 3 \mathrm{H}), 1.91(\mathrm{~s}, 3 \mathrm{H}) ;{ }^{13} \mathbf{C}$ NMR (100 MHz,

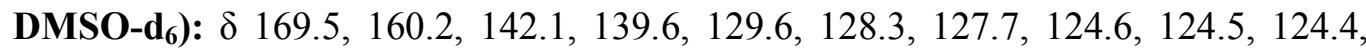
121.0, 119.3, 109.5, 96.5, 23.4, 14.0; IR (neat, $\mathbf{c m}^{-1}$ ): $v$ 3283, 3027, 1673, 1524, 1239 , 942, 742; MS (m/z, ESI): 280, 221; HRMS calculated for $\mathrm{C}_{18} \mathrm{H}_{18} \mathrm{NO}_{2}(\mathrm{M}+\mathrm{H})^{+}$: 280.1332; Found: 280.1333; $\mathbf{R}_{\mathbf{f}}=0.6$ (DCM: EtOAc, 10:1).

NOESY (400 MHz, DMSO-d $\mathbf{d}_{\mathbf{6}}$ ). The copy of NOESY spectra is shown in page 53.<smiles>CC(C)(NC1(c2ccccc2)Oc2cccc3c2C1=CCCC3)c1ccccc1</smiles> 


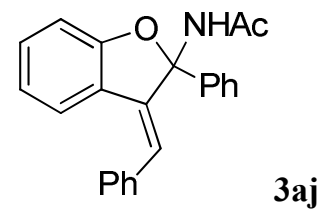

Yield 81\% (55 mg); white solid; m.p. $128-129{ }^{\circ} \mathrm{C} ;{ }^{1} \mathbf{H}$ NMR (400 MHz, DMSO-d 6 ): $\delta 9.10(\mathrm{~s}, 1 \mathrm{H}), 7.53(\mathrm{~d}, J=8.0 \mathrm{~Hz}, 2 \mathrm{H}), 7.43-7.29(\mathrm{~m}, 8 \mathrm{H}), 7.25(\mathrm{t}, J=7.6 \mathrm{~Hz}, 1 \mathrm{H})$, $7.18(\mathrm{~d}, J=7.6 \mathrm{~Hz}, 1 \mathrm{H}), 6.99(\mathrm{~d}, J=8.4 \mathrm{~Hz}, 1 \mathrm{H}), 6.75(\mathrm{t}, J=7.6 \mathrm{~Hz}, 1 \mathrm{H}), 6.59(\mathrm{~s}$, 1H), 1.97(s, 3H); ${ }^{13}$ C NMR (100 MHz, DMSO-d $)$ ): $\delta$ 169.7, 161.1, 141.4, 140.4, $136.1,130.8,128.53,128.50,128.32,128.13,127.7,124.9,123.2,123.0,122.5,120.5$, 110.0, 97.1, 23.4; IR (neat, $\left.\mathbf{c m}^{-1}\right):$ v 3302, 2920, 2852, 1674, 1522, 1461, 1185, 754, 697; MS (m/z, ESI): 364, 283; HRMS calculated for $\mathrm{C}_{23} \mathrm{H}_{19} \mathrm{NO}_{2} \mathrm{Na}(\mathrm{M}+\mathrm{Na})^{+}$: 364.1308; Found: 364.1308; $\mathbf{R}_{\mathbf{f}}=0.4$ (PE: EtOAc, 1:1).

\section{Mechanistic studies}

\section{Control Experiments}

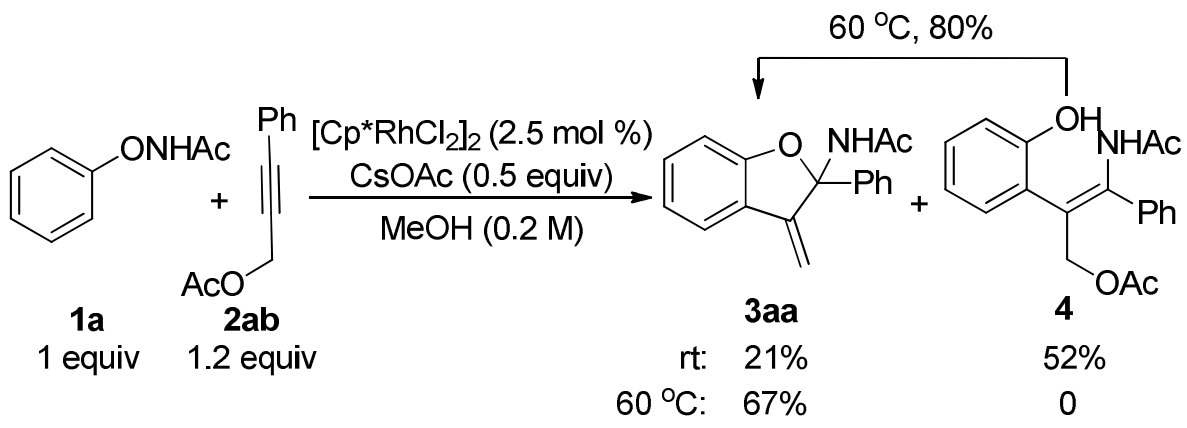

Without any particular precautions to extrude oxygen or moisture, the phenyloxyacetamide 1a (45.3 mg, $0.3 \mathrm{mmol}, 1$ equiv), 3-phenylprop-2-yn-1-yl acetate 2ab (62.6 mg, $0.36 \mathrm{mmol}, 1.2$ equiv), $\left[\mathrm{Cp}^{*} \mathrm{RhCl}_{2}\right]_{2}(4.8 \mathrm{mg}, 0.0075 \mathrm{mmol}, 2.5 \mathrm{~mol} \%)$ and $\mathrm{CsOAc}$ (30 mg, $0.15 \mathrm{mmol}, 0.5$ equiv) were weighted in a $4 \mathrm{~mL}$ vial equipped with a stir bar. $\mathrm{MeOH}(1.5 \mathrm{~mL}, 0.2 \mathrm{M})$ was then added. The reaction was stirred at room temperature or $60{ }^{\circ} \mathrm{C}$, and monitored by TLC. Afterwards, two reaction mixtures were diluted with EtOAc separately transferred to round bottom flasks. Silica gel was added to the flasks and volatiles were evaporated under reduced pressure. The purification was performed by flash column chromatography on silica gel to afford 3aa (17 mg, 21\%) and $4(51 \mathrm{mg}, 52 \%)$. 
Without any particular precautions to extrude oxygen or moisture, the ortho-hydroxyphenyl substituted enamide $4(16.3 \mathrm{mg}, 0.05 \mathrm{mmol})$ was weighted in a $4 \mathrm{~mL}$ vial equipped with a stir bar. $\mathrm{MeOH}(0.2 \mathrm{M})$ was then added. The reaction was stirred at $60{ }^{\circ} \mathrm{C}$ and monitored by TLC. Afterwards, it was diluted with EtOAc and transferred to a round bottom flask. Silica gel was added to the flask and volatiles were evaporated under reduced pressure. The purification was performed by flash column chromatography on silica gel to give 3aa (10.6 mg) in $80 \%$ yield.<smiles>CC(=O)N/C(=C(\COC(C)=O)c1ccccc1)c1ccccc1</smiles>

Yield 52\% (51 mg); white solid; m.p. 140-141 ${ }^{\circ} \mathrm{C} ;{ }^{1}$ H NMR (400 MHz, DMSO-d 6 ): $\delta 9.56(\mathrm{~s}, 1 \mathrm{H}), 9.07(\mathrm{~s}, 1 \mathrm{H}), 7.40-7.33(\mathrm{~m}, 5 \mathrm{H}), 7.14-7.09(\mathrm{~m}, 2 \mathrm{H}), 6.87(\mathrm{~d}, J=7.6 \mathrm{~Hz}$, 1H), $6.79(\mathrm{t}, J=7.2 \mathrm{~Hz}, 1 \mathrm{H}), 4.76(\mathrm{~s}, 2 \mathrm{H}), 1.91(\mathrm{~s}, 3 \mathrm{H}), 1.68(\mathrm{~s}, 3 \mathrm{H}) ;{ }^{13} \mathrm{C}$ NMR (100 MHz, DMSO-d $\left.\mathbf{d}_{\mathbf{6}}\right): \delta 170.2,168.5,154.5,138.0,137.7,130.7,128.7,128.5,127.9$, 126.7, 124.9, 118.9, 115.9, 63.7, 22.6, 20.8; IR (neat, $\mathbf{c m}^{-1}$ ): v 3270, 2923, 1707, 1653, 1521, 1446, 1377, 1251, 1021, 750, 704; MS (m/z, ESI): 348, 266, 207;

HRMS calculated for $\mathrm{C}_{19} \mathrm{H}_{19} \mathrm{NaNO}_{4}(\mathrm{M}+\mathrm{Na})^{+}: 348.1206$; Found: $348.1201 ; \mathbf{R}_{\mathbf{f}}=0.2$ (PE: EtOAc, 1:1).

\section{Deuteration Experiments}

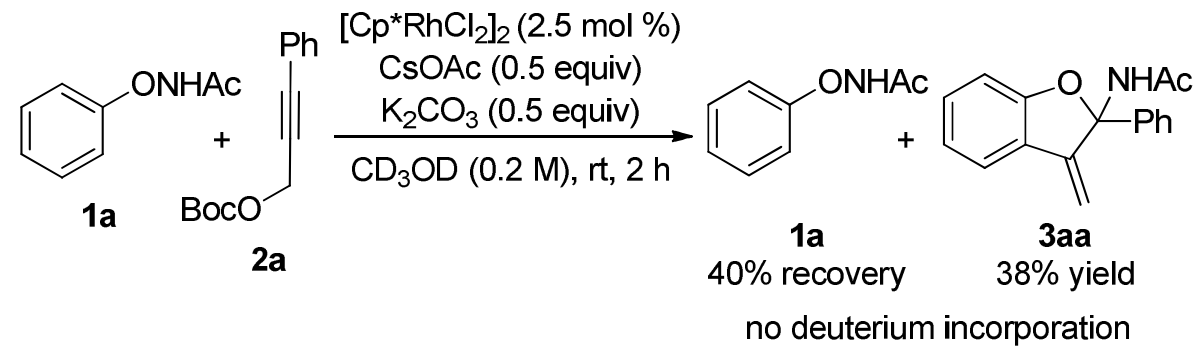

Without any particular precautions to extrude oxygen or moisture, the phenyloxyacetamide $1 \mathrm{a} \quad(30.2 \mathrm{mg}, \quad 0.2 \mathrm{mmol}, 1$ equiv), tert-butyl (3-phenylprop-2-yn-1-yl) carbonate $2 \mathbf{a}\left(55.7 \mathrm{mg}, 0.24 \mathrm{mmol}, 1.2 \text { equiv), [Cp* } \mathrm{RhCl}_{2}\right]_{2}$ (3.2 mg, $0.005 \mathrm{mmol}, 2.5 \mathrm{~mol} \%$ ), $\mathrm{CsOAc}\left(19.2 \mathrm{mg}, 0.1 \mathrm{mmol}, 0.5\right.$ equiv) and $\mathrm{K}_{2} \mathrm{CO}_{3}$ 
(13.8 mg, $0.1 \mathrm{mmol}, 0.5$ equiv) were weighted in a $4 \mathrm{~mL}$ vial equipped with a stir bar. $\mathrm{CD}_{3} \mathrm{OD}(0.2 \mathrm{M})$ was then added. The reaction was stirred at room temperature for 2 hours. Afterwards, it was diluted with EtOAc and transferred to a round bottom flask. Silica gel was added to the flask and volatiles were evaporated under reduced pressure. After purification by flash column chromatography, 3aa was isolated in $38 \%$ yield (20 $\mathrm{mg})$ and $40 \%$ of $1 \mathrm{a}(12 \mathrm{mg})$ was recovered, no deuterium incorporation was found in both of them.

\section{KIE experiments}

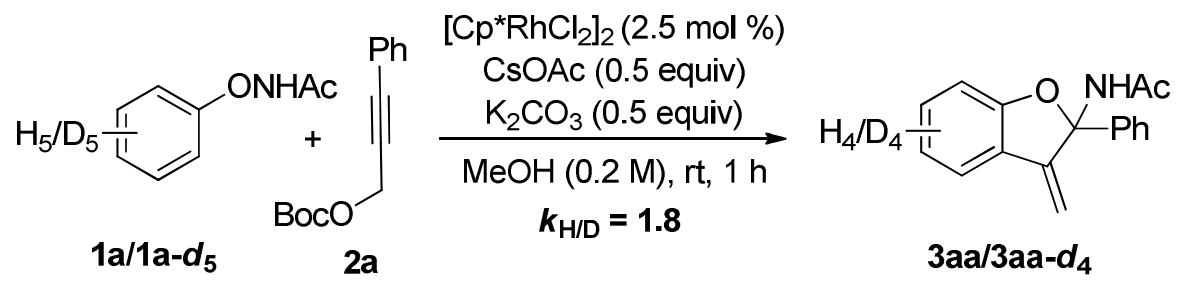

Intermolecular KIE experiment was performed. Without any particular precautions to extrude oxygen or moisture, the phenyloxyacetamide $1 \mathrm{a}(15.1 \mathrm{mg}, 0.1 \mathrm{mmol}, 1$ equiv), 1a-d 5 (15.6 mg, $0.1 \mathrm{mmol}, 1$ equiv), tert-butyl (3-phenylprop-2-yn-1-yl) carbonate $2 \mathrm{a}\left(27.8 \mathrm{mg}, 0.12 \mathrm{mmol}, 1.2 \text { equiv), [Cp* } \mathrm{RhCl}_{2}\right]_{2}(1.6 \mathrm{mg}, 0.0025 \mathrm{mmol}$, $2.5 \mathrm{~mol} \%$ ), CsOAc (10 mg, $0.05 \mathrm{mmol}, 0.5$ equiv) and $\mathrm{K}_{2} \mathrm{CO}_{3}(6.9 \mathrm{mg}, 0.05 \mathrm{mmol}$, 0.5 equiv) were weighted in a $4 \mathrm{~mL}$ vial equipped with a stir bar. $\mathrm{MeOH}(0.2 \mathrm{M})$ was then added. The reaction was stirred at room temperature for 1 hour. Afterwards, it was diluted with EtOAc and transferred to a round bottom flask. Silica gel was added to the flask and volatiles were evaporated under reduced pressure. After purification by flash column chromatography, 3aa and $\mathbf{3 a a}-\boldsymbol{d}_{4}$ were isolated as inseparable mixture. The KIE was determined to be $\mathrm{k}_{H / D}=1.8$. 


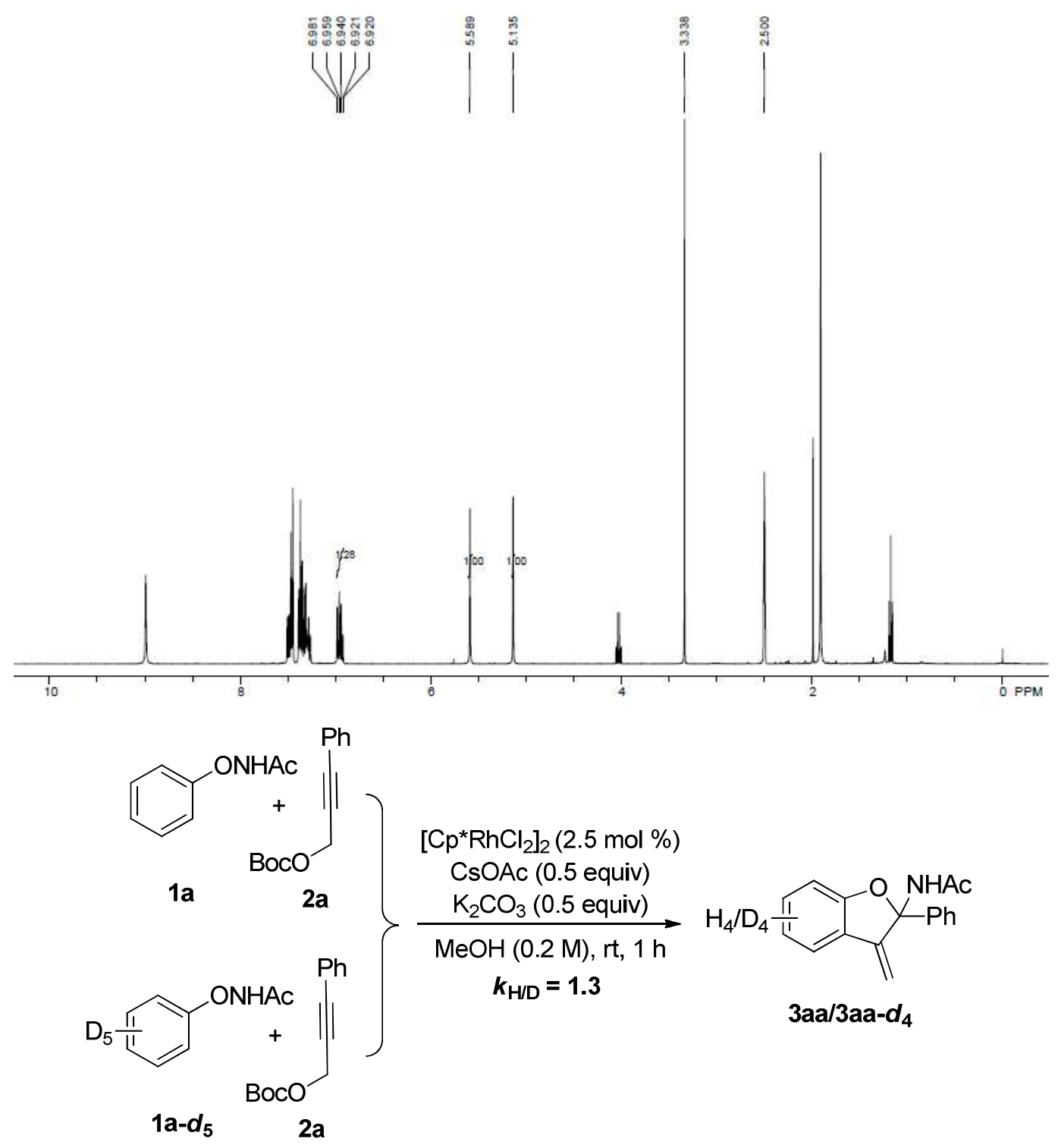

Independent side-by-side experiments were performed. Without any particular precautions to extrude oxygen or moisture, the phenyloxyacetamide 1a (15.1 mg, 0.1 mmol, 1 equiv) or $\mathbf{1 a - d _ { 5 }}(15.6 \mathrm{mg}, 0.1 \mathrm{mmol}, 1$ equiv), tert-butyl (3-phenylprop-2-yn-1-yl) carbonate $\mathbf{2 a}\left(27.8 \mathrm{mg}, 0.12 \mathrm{mmol}, 1.2 \text { equiv), [Cp* } \mathrm{RhCl}_{2}\right]_{2}$ (1.6 mg, $0.0025 \mathrm{mmol}, 2.5 \mathrm{~mol} \%$ ), CsOAc (10 mg, $0.05 \mathrm{mmol}, 0.5$ equiv) and $\mathrm{K}_{2} \mathrm{CO}_{3}$ (6.9 mg, $0.05 \mathrm{mmol}, 0.5$ equiv) were weighted in two separated vials equipped with a stir bar. $\mathrm{MeOH}(0.2 \mathrm{M})$ was then added. The two reactions were stirred side by side at room temperature for 1 hour. Afterwards, two reaction mixtures were filtered separately through Celite, washed with $10 \mathrm{~mL}$ of DCM. The solvent was then removed under reduced pressure and ${ }^{1} \mathrm{H}$ NMR was taken for these two samples 
separately using trimethoxybenzene $(5.6 \mathrm{mg})$ as the internal standard. 3aa was obtained in $34 \%$ yield and 3aa- $\boldsymbol{d}_{4}$ was obtained in $26 \%$ yield. Thus the KIE was found to be 1.3 .

Crude ${ }^{1} \mathrm{H}$ NMR of the reaction of $\mathbf{1 a}$ and $\mathbf{2 a}$ under standard conditions for $1 \mathrm{~h}$
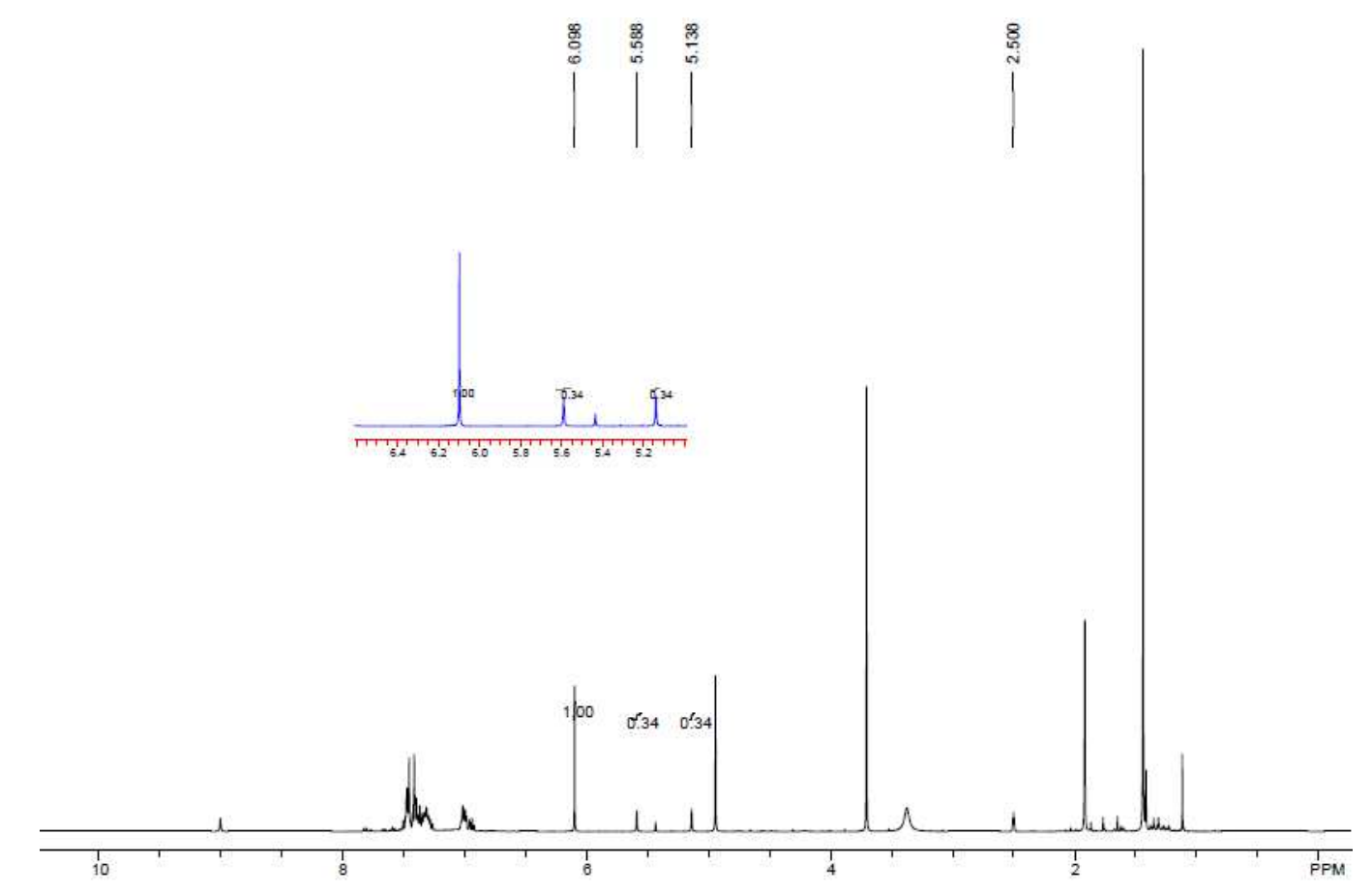

Crude ${ }^{1} \mathrm{H}$ NMR of the reaction of $\mathbf{1 a}-\boldsymbol{d}_{5}$ and $\mathbf{2 a}$ under standard conditions for $1 \mathrm{~h}$

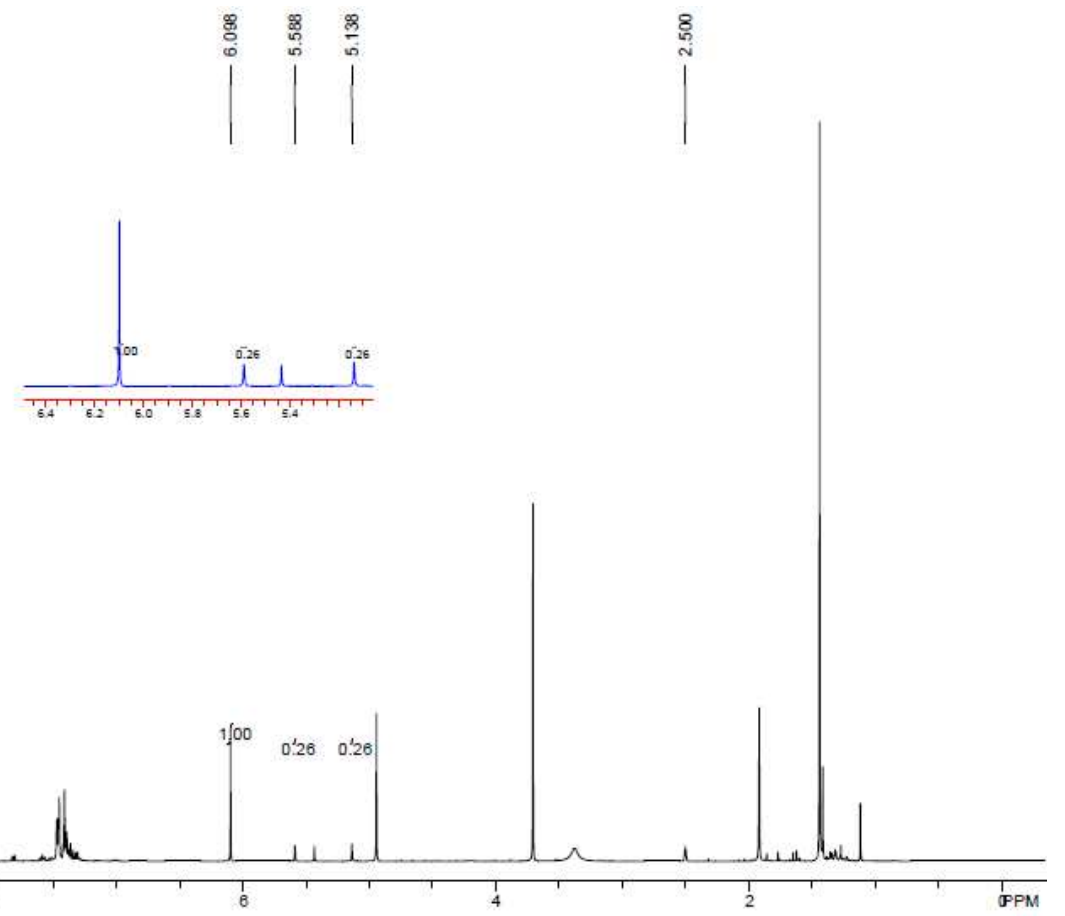




\section{Gram Scale Synthesis and Derivatization of 3aa}

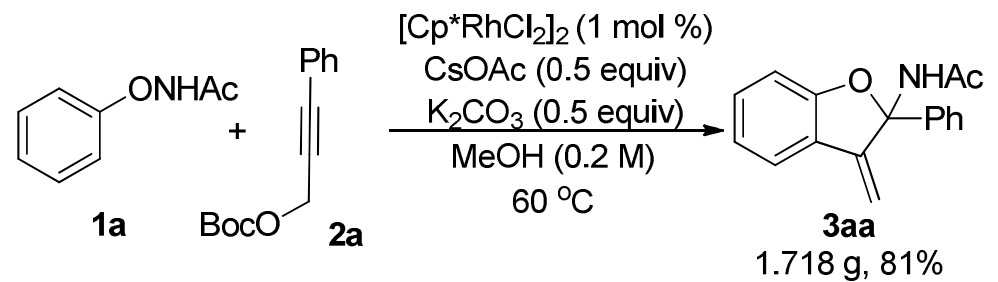

Without any particular precautions to extrude oxygen or moisture, the phenyloxyacetamide 1a $(1.208 \mathrm{~g}, 8 \mathrm{mmol}, 1$ equiv), tert-butyl (3-phenylprop-2-yn-1-yl) carbonate $2 \mathbf{a}\left(2.042 \mathrm{~g}, 8.8 \mathrm{mmol}, 1.1 \text { equiv), [Cp* } \mathrm{RhCl}_{2}\right]_{2}$ (49.4 mg, 0.08 mmol, 1 mol \%), CsOAc (0.768 g, 4 mmol, 0.5 equiv) and $\mathrm{K}_{2} \mathrm{CO}_{3}$ ( $0.552 \mathrm{~g}, 4 \mathrm{mmol}, 0.5$ equiv) were weighted in a $100 \mathrm{~mL}$ tube equipped with a stir bar. $\mathrm{MeOH}(0.2 \mathrm{M})$ was then added. The reaction was stirred at $60{ }^{\circ} \mathrm{C}$ and monitored by TLC. Afterwards, it was diluted with EtOAc and transferred to a round bottom flask. Silica gel was added to the flask and volatiles were evaporated under reduced pressure. The purification was performed by flash column chromatography on silica gel to afford 3aa $(1.718 \mathrm{~g}, 81 \%)$.

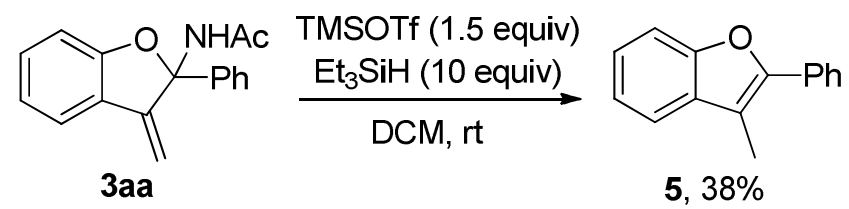

Without any particular precautions to extrude oxygen or moisture, 3aa (26.5 mg, $0.1 \mathrm{mmol}, 1$ equiv) was dissolved in DCM $(0.1 \mathrm{M})$ in a $4 \mathrm{ml}$ vial equipped with a stir bar, followed by the addition of $\mathrm{Et}_{3} \mathrm{SiH}(160 \mu \mathrm{l}, 1 \mathrm{mmol}, 10$ equiv) and TMSOTf (30 $\mu 1,0.15$ mmol, 1.5 equiv). The mixture was stirred at room temperature and monitored by TLC. Afterwards, it was diluted with EtOAc and transferred to a round bottom flask. Silica gel was added to the flask and volatiles were evaporated under reduced pressure. The purification was performed by flash column chromatography on silica gel to afford 5 ( $8 \mathrm{mg}, 38 \%)$.

5 is a known compound and all data were in agreement with those reported. ${ }^{1}$ 


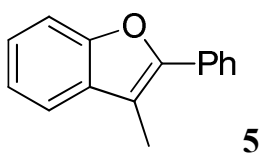

Yield 38\%; white solid; ${ }^{\mathbf{1}} \mathbf{H}$ NMR (400 MHz, $\left.\mathbf{C D C l}_{3}\right): \delta 7.82(\mathrm{~d}, J=8.0 \mathrm{~Hz}, 2 \mathrm{H}), 7.54$ (d, $J=8.4 \mathrm{~Hz}, 1 \mathrm{H}), 7.50-7.46(\mathrm{~m}, 3 \mathrm{H}), 7.36(\mathrm{t}, J=7.6 \mathrm{~Hz}, 1 \mathrm{H}), 7.32-7.23(\mathrm{~m}, 2 \mathrm{H})$, 2.49 (s, 3H); ${ }^{13} \mathbf{C}$ NMR (100 MHz, $\left.\mathbf{C D C l}_{3}\right): \delta 153.9,150.8,131.6,131.3,128.8$, 128.0, 126.8, 124.4, 122.5, 119.4, 111.4, 111.1, 9.6; IR (neat, $\mathbf{c m}^{-1}$ ): v 3059, 2924, 1457, 1362, 743, 693; MS (m/z, EI): 208, 178, 152, 131; $\mathbf{R}_{\mathbf{f}}=0.7$ in PE.

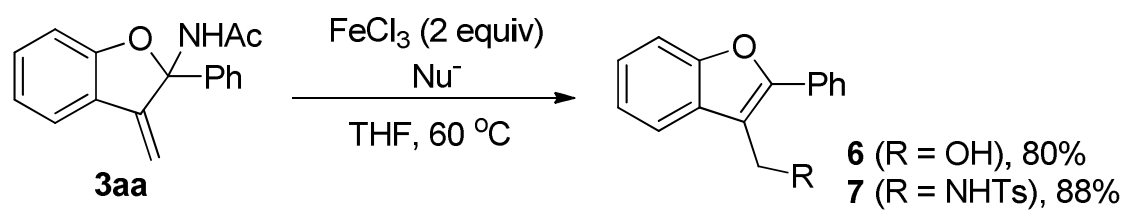

Without any particular precautions to extrude oxygen or moisture, 3aa $(26.5 \mathrm{mg}$, $0.1 \mathrm{mmol}, 1$ equiv) and $\mathrm{FeCl}_{3}$ (32.4 mg, $0.2 \mathrm{mmol}, 2$ equiv) were weighted in a $4 \mathrm{ml}$ vial equipped with a stir bar, followed by the addition of $\mathrm{H}_{2} \mathrm{O}$ (50 equiv) or $\mathrm{TsNH}_{2}$ ( $85.5 \mathrm{mg}, 0.5 \mathrm{mmol}, 5$ equiv). THF $(0.2 \mathrm{M})$ was then added. The mixture was stirred at $60{ }^{\circ} \mathrm{C}$ and monitored by TLC. Afterwards, it was diluted with EtOAc and transferred to a round bottom flask. Silica gel was added to the flask and volatiles were evaporated under reduced pressure. The purification was performed by flash column chromatography on silica gel to give $6(18 \mathrm{mg}, 80 \%)$ or 7 (33 mg, 88\%).

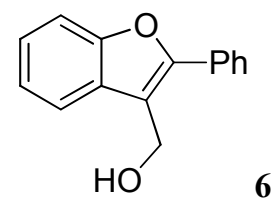

Yield 80\%; white solid; m.p. $77-78{ }^{\circ} \mathrm{C} ;{ }^{1} \mathbf{H}$ NMR (400 MHz, DMSO-d 6 ): $\delta 7.88(\mathrm{~d}, J$ $=8.4 \mathrm{~Hz}, 2 \mathrm{H}), 7.79\left(\mathrm{dd}, J_{I}=6.8 \mathrm{~Hz}, J_{2}=0.8 \mathrm{~Hz}, 1 \mathrm{H}\right), 7.61(\mathrm{~d}, J=8.4 \mathrm{~Hz}, 1 \mathrm{H}), 7.56$ (t, $J=7.6 \mathrm{~Hz}, 2 \mathrm{H}), 7.47$ (t, $J=7.6 \mathrm{~Hz}, 1 \mathrm{H}), 7.37-7.27(\mathrm{~m}, 2 \mathrm{H}), 5.36(\mathrm{t}, J=5.2 \mathrm{~Hz}$, $1 \mathrm{H}), 4.77(\mathrm{~d}, J=5.2 \mathrm{~Hz}, 2 \mathrm{H}) ;{ }^{13} \mathbf{C}$ NMR (100 MHz, DMSO-d $\left.\mathbf{6}\right): \delta 153.3,151.9$, $129.9,129.5,129.0,128.9,127.0,124.7,122.8,120.5,116.4,111.0,53.4$; IR (neat, $\left.\mathbf{c m}^{-1}\right):$ v 3374, 2938, 1453, 1121, 1069, 744, 694; MS (m/z, ESI): 266, 207; HRMS calculated for $\mathrm{C}_{17} \mathrm{H}_{16} \mathrm{NO}_{2}\left(\mathrm{M}+\mathrm{NH}_{4}\right)^{+}$: 266.1176; Found: 266.1177; $\mathbf{R}_{\mathbf{f}}=0.5(\mathrm{PE}$ : 
EtOAc, 5:1).

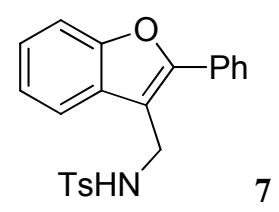

Yield 88\%; white solid; m.p. 139-141 ${ }^{\circ} \mathrm{C} ;{ }^{1}$ H NMR (400 MHz, DMSO-d $\left.{ }_{6}\right): \delta 8.13$ (t, $J=5.6 \mathrm{~Hz}, 1 \mathrm{H}), 7.69-7.65(\mathrm{~m}, 4 \mathrm{H}), 7.57(\mathrm{~d}, J=8.8 \mathrm{~Hz}, 2 \mathrm{H}), 7.49-7.44(\mathrm{~m}, 3 \mathrm{H})$, 7.36-7.27 (m, 3H), $7.25(\mathrm{t}, J=7.6 \mathrm{~Hz}, 1 \mathrm{H}), 4.21(\mathrm{~d}, J=5.6 \mathrm{~Hz}, 2 \mathrm{H}), 2.39(\mathrm{~s}, 3 \mathrm{H}) ;{ }^{13} \mathbf{C}$ NMR (100 MHz, DMSO-d $\left.{ }_{6}\right): \delta$ 153.3, 152.2, 142.8, 137.1, 129.5, 129.4, 129.1, $128.9,128.8,127.2,126.7,124.9,122.9,120.2,111.5,111.0,37.0,21.0$; IR (neat, $\left.\mathbf{c m}^{-1}\right): v 3284,2924,2854,1340,1158,1039,818,747,671 ;$ MS (m/z, ESI): 395, 306, 207; HRMS calculated for $\mathrm{C}_{22} \mathrm{H}_{23} \mathrm{~N}_{2} \mathrm{O}_{3} \mathrm{~S}\left(\mathrm{M}+\mathrm{NH}_{4}\right)^{+}$: 395.1424; Found: 395.1430; $\mathbf{R}_{\mathbf{f}}=0.4(\mathrm{PE}:$ EtOAc, $5: 1)$.

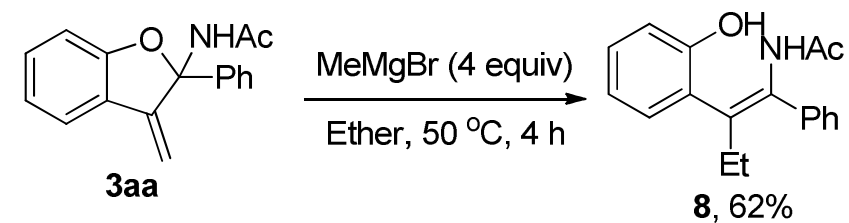

In a reaction flask, 3aa $\left(39.8 \mathrm{mg}, 0.15 \mathrm{mmol}\right.$, 1equiv) was dissolved in $\mathrm{Et}_{2} \mathrm{O}(0.3$ $\mathrm{M})$, followed by the addition of $\mathrm{MeMgBr}(3 \mathrm{M}$ in ether, $0.2 \mathrm{ml}, 0.6 \mathrm{mmol}, 4$ equiv). The mixture was stirred at $50{ }^{\circ} \mathrm{C}$ for 4 hours under $\mathrm{N}_{2}$ atmosphere. Afterwards, it was quenched with aqueous $\mathrm{HCl}(1 \mathrm{M})$ solution and extracted with $\mathrm{Et}_{2} \mathrm{O}(3 \mathrm{x})$. The organic phase was washed with brine after which it was dried over $\mathrm{Na}_{2} \mathrm{SO}_{4}$, filtered and evaporated under reduced pressure. The crude product was purified by flash column chromatography on silica gel to afford $8(26 \mathrm{mg}, 62 \%)$.<smiles>CC/C(=C(\NC(C)=O)c1ccccc1)c1ccccc1O</smiles>

Yield 62\%; light yellow solid; m.p. $148-150{ }^{\circ} \mathrm{C} ;{ }^{1} \mathbf{H}$ NMR (400 MHz, DMSO-d $\left.{ }_{6}\right): \delta$ $9.24(\mathrm{~s}, 1 \mathrm{H}), 9.15(\mathrm{~s}, 1 \mathrm{H}), 7.04-6.93(\mathrm{~m}, 6 \mathrm{H}), 6.72(\mathrm{~d}, J=8.0 \mathrm{~Hz}, 1 \mathrm{H}), 6.67\left(\mathrm{dd}, J_{l}=\right.$ 
$\left.7.2 \mathrm{~Hz}, J_{2}=1.2 \mathrm{~Hz}, 1 \mathrm{H}\right), 6.54(\mathrm{t}, J=7.6 \mathrm{~Hz}, 1 \mathrm{H}), 2.43(\mathrm{q}, J=7.6 \mathrm{~Hz}, 2 \mathrm{H}), 1.98(\mathrm{~s}$, 3H), 0.84 (t, $J=7.6 \mathrm{~Hz}, 3 \mathrm{H}) ;{ }^{13} \mathbf{C}$ NMR (100 MHz, CD $\left.\mathbf{C l}\right): \delta 170.8,153.1,137.4$, $137.3,131.0,129.7,129.1,128.9,128.1,127.9,126.0,119.2,115.7,28.1,23.1,11.1$; IR (neat, $\mathbf{c m}^{-1}$ ): $v$ 3353, 3198, 2992, 2935, 1638, 1484, 1458, 1443, 1369, 1236, 972, 762, 745, 693; MS (m/z, ESI): 282, 223; HRMS calculated for $\mathrm{C}_{18} \mathrm{H}_{20} \mathrm{NO}_{2}(\mathrm{M}+\mathrm{H})^{+}$: 282.1489; Found: 282.1490; $\mathbf{R}_{\mathbf{f}}=0.2$ (DCM: EtOAc, 10:1).

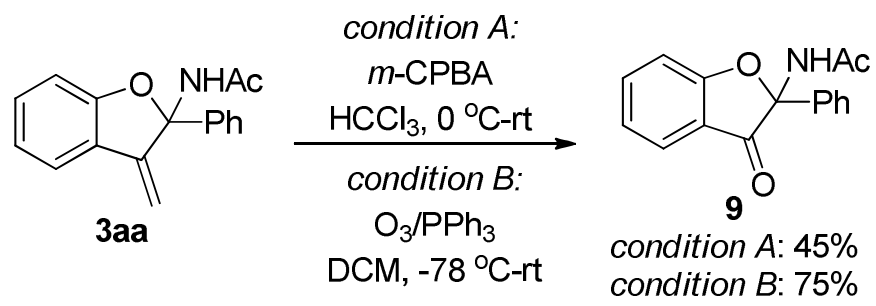

Condition A: Without any particular precautions to extrude oxygen or moisture, 3aa (26.5 mg, $0.1 \mathrm{mmol}, 1$ equiv) was dissolved in $\operatorname{HCCl}_{3}(0.1 \mathrm{M})$ in a $4 \mathrm{ml}$ vial equipped with a stir bar, the resulting solution was cooled to $0{ }^{\circ} \mathrm{C}$, followed by the addition of $m$-CPBA (24.4 mg, $0.12 \mathrm{mmol}, 1.2$ equiv). The mixture was then allowed to react at room temperature and monitored by TLC. Afterwards, it was diluted with EtOAc and transferred to a round bottom flask. Silica gel was added to the flask and volatiles were evaporated under reduced pressure. The purification was performed by flash column chromatography on silica gel to give 9 (12 $\mathrm{mg}, 45 \%)$.

Condition B: Without any particular precautions to extrude oxygen or moisture, 3aa (53 mg, $0.2 \mathrm{mmol}, 1$ equiv) was dissolved in DCM (0.04 M) in a reaction flask equipped with a stir bar, the resulting solution was cooled to $-78{ }^{\circ} \mathrm{C}$. Ozone was bubbled through the solution for 2 mins and $\mathrm{PPh}_{3}(68 \mathrm{mg}, 0.26 \mathrm{mmol}, 1.3$ equiv) was then added. After slow warm to room temperature, the reaction mixture was stirred for 4 hours and monitored by TLC. Afterwards, it was diluted with EtOAc and transferred to a round bottom flask. Silica gel was added to the flask and volatiles were evaporated under reduced pressure. The purification was performed by flash column chromatography on silica gel to give 9 (40 mg, 75\%). 


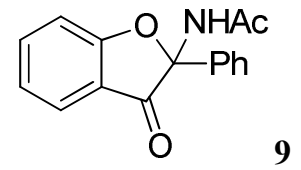

Yield 75\%; white solid; m.p. 188-190 ${ }^{\circ} \mathrm{C} ;{ }^{1} \mathbf{H}$ NMR (400 MHz, $\mathbf{C D}_{3} \mathbf{C l )}$ ) $\delta$ 7.69-7.59 (m, 4H), 7.37-7.35 (m, 3H), $7.17(\mathrm{~d}, J=8.8 \mathrm{~Hz}, 1 \mathrm{H}), 7.07$ (t, $J=7.2 \mathrm{~Hz}, 1 \mathrm{H}), 6.82(\mathrm{~s}$, 1H), 1.84 (s, 3H); ${ }^{13}$ C NMR (100 MHz, CD Cl): $\delta$ 195.0, 170.4, 169.6, 137.9, 134.5, 129.8, 129.1, 125.8, 125.2, 122.4, 120.0, 112.4, 91.7, 22.2; IR (neat, $\mathbf{c m}^{-1}$ ): v 3250, 3028, 2919, 1738, 1664, 1613, 1533, 1279, 969, 759, 698; MS (m/z, ESI): 268, 209;

HRMS calculated for $\mathrm{C}_{16} \mathrm{H}_{14} \mathrm{NO}_{3}(\mathrm{M}+\mathrm{H})^{+}: 268.0968$; Found: $268.0975 ; \mathbf{R}_{\mathbf{f}}=0.5$ (PE: EtOAc, 1:1).

\section{References}

(1) Liu, G.; Shen, Y.; Zhou, Z.; Lu, X. Angew. Chem. Int. Ed. 2013, 52, 6033.

(2) Nibbs, A. E.; Montgomery, T. D.; Zhu, Y.; Rawal, V. H. J. Org. Chem. 2015, 80, 4928.

(3) (a) Chen, Z.-S.; Duan, X.-H.; Wu, L.-Y.; Ali, S.; Ji, K.-G.; Zhou, P.-X.; Liu, X.-Y.; Liang, Y.-M. Chem. Eur. J. 2011, 17, 6918. (b) Nishimoto, Y.; Okita, A.; Yasuda, M.; Baba, A. Org. Lett. 2012, 14, 1846. (c) Gu, Y.; Chang, D.; Leng, X.; Gu, Y.; Shen, Q. Organometallics 2015, 34, 3065. 
VII Copies of ${ }^{1} \mathrm{H},{ }^{13} \mathrm{C}$ NMR spectra
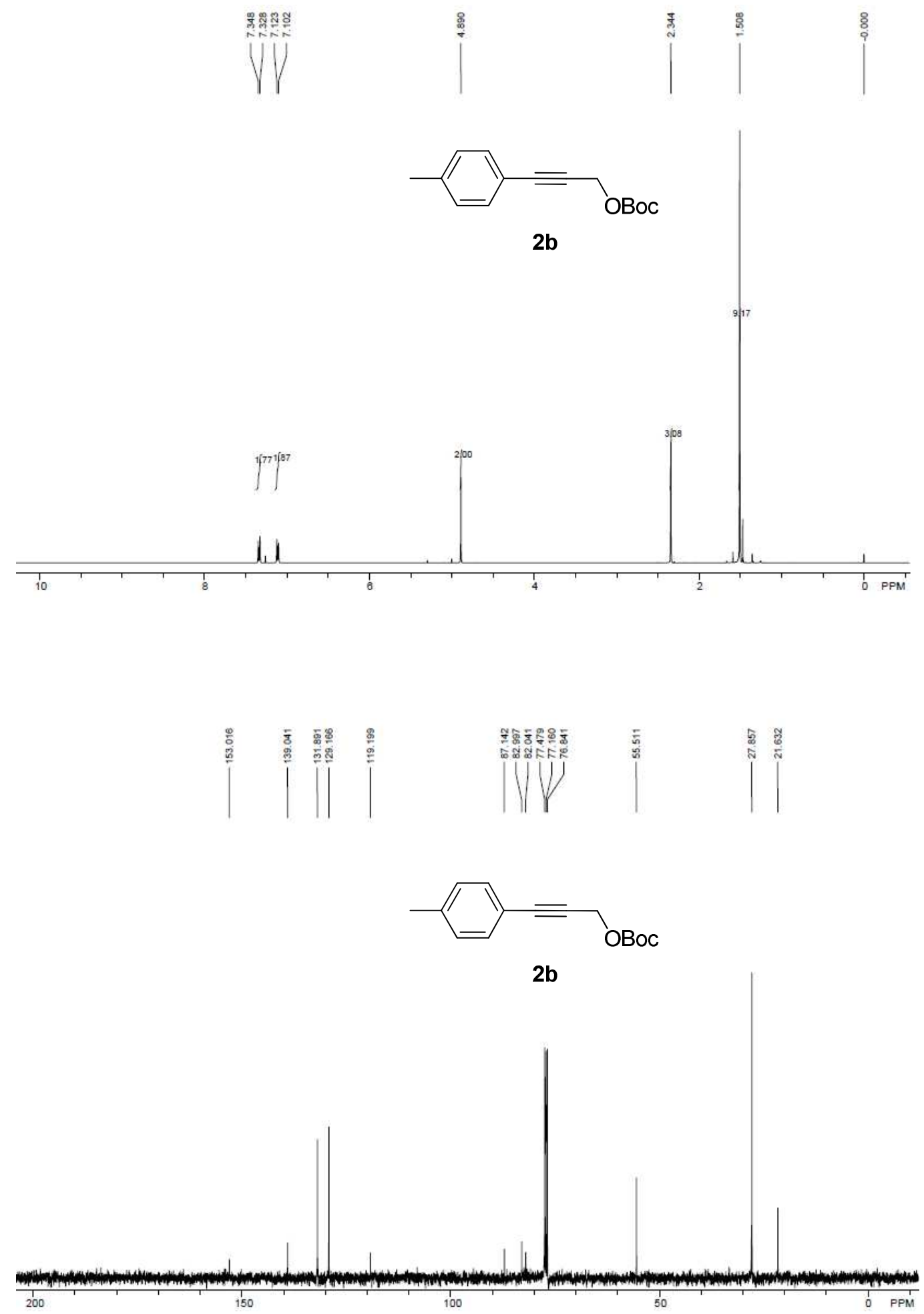


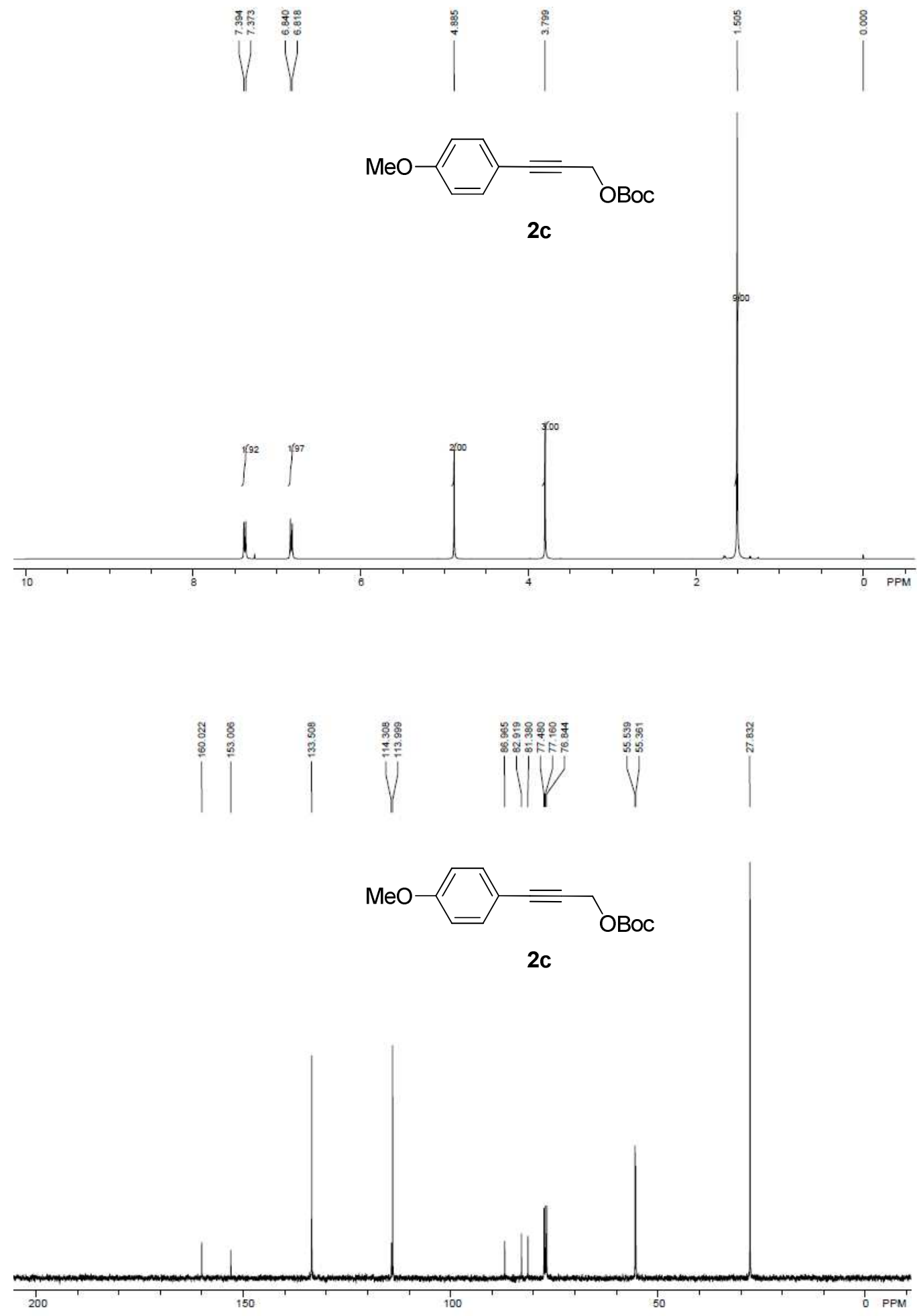




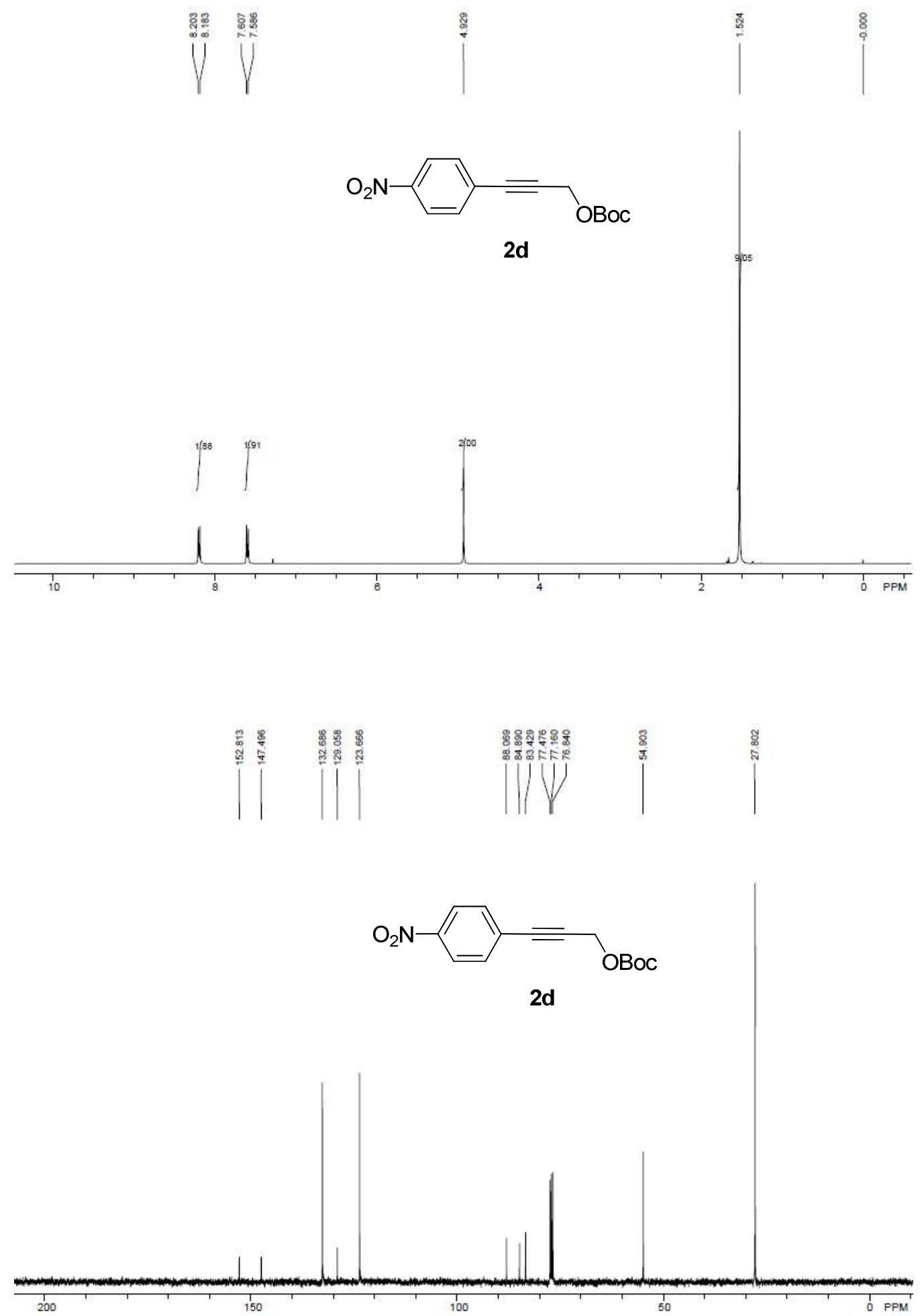



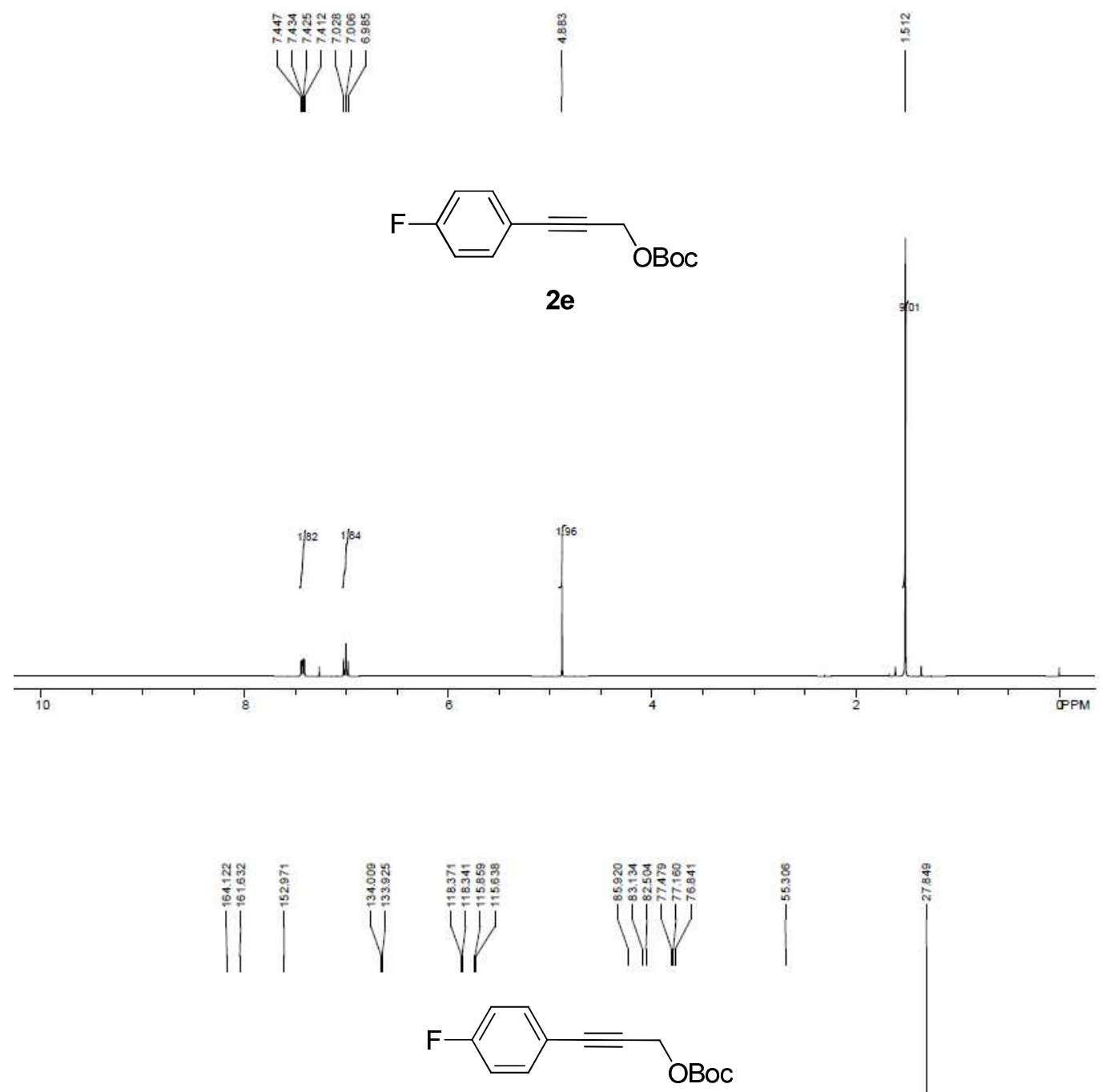

200 150 


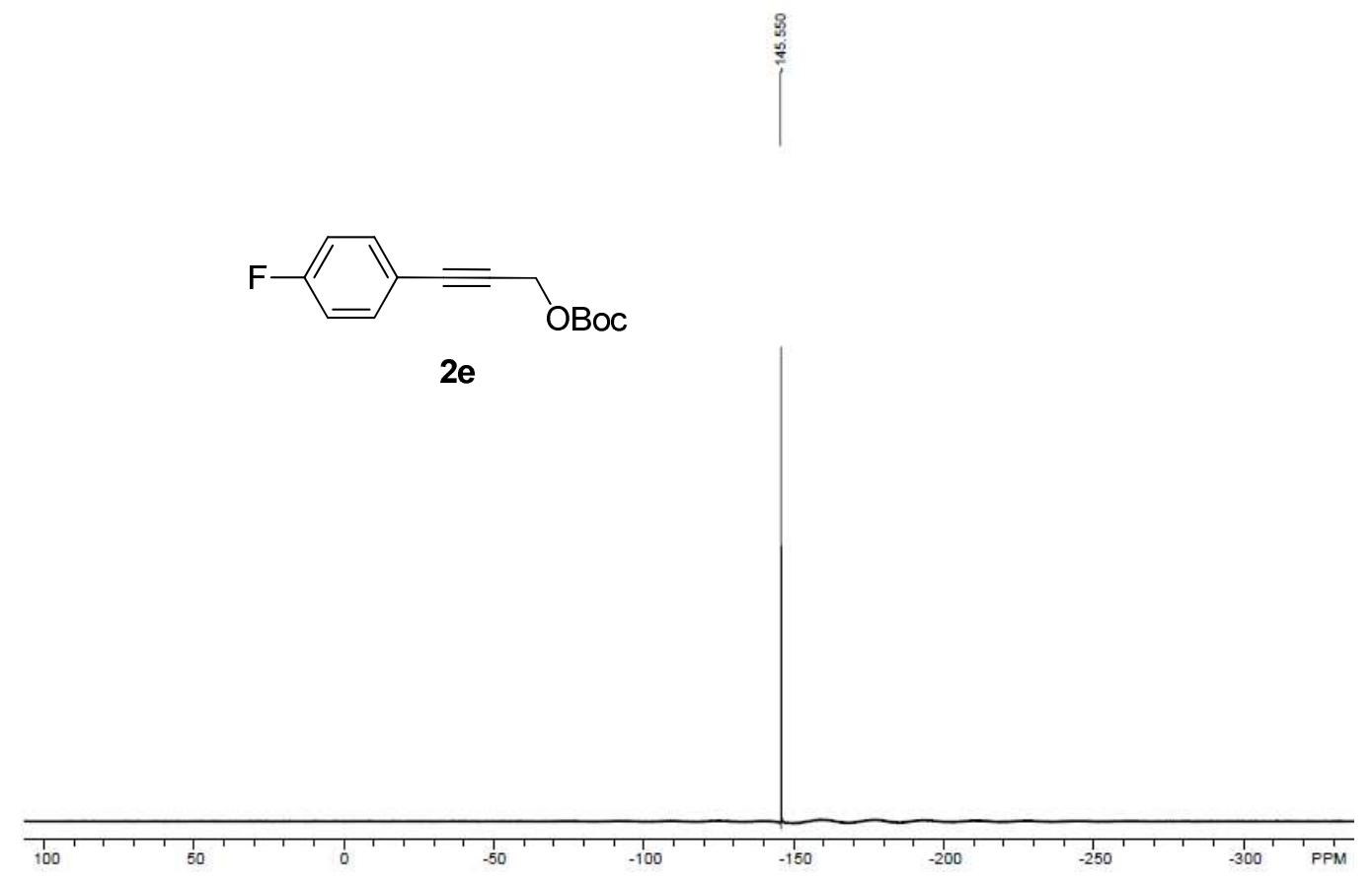




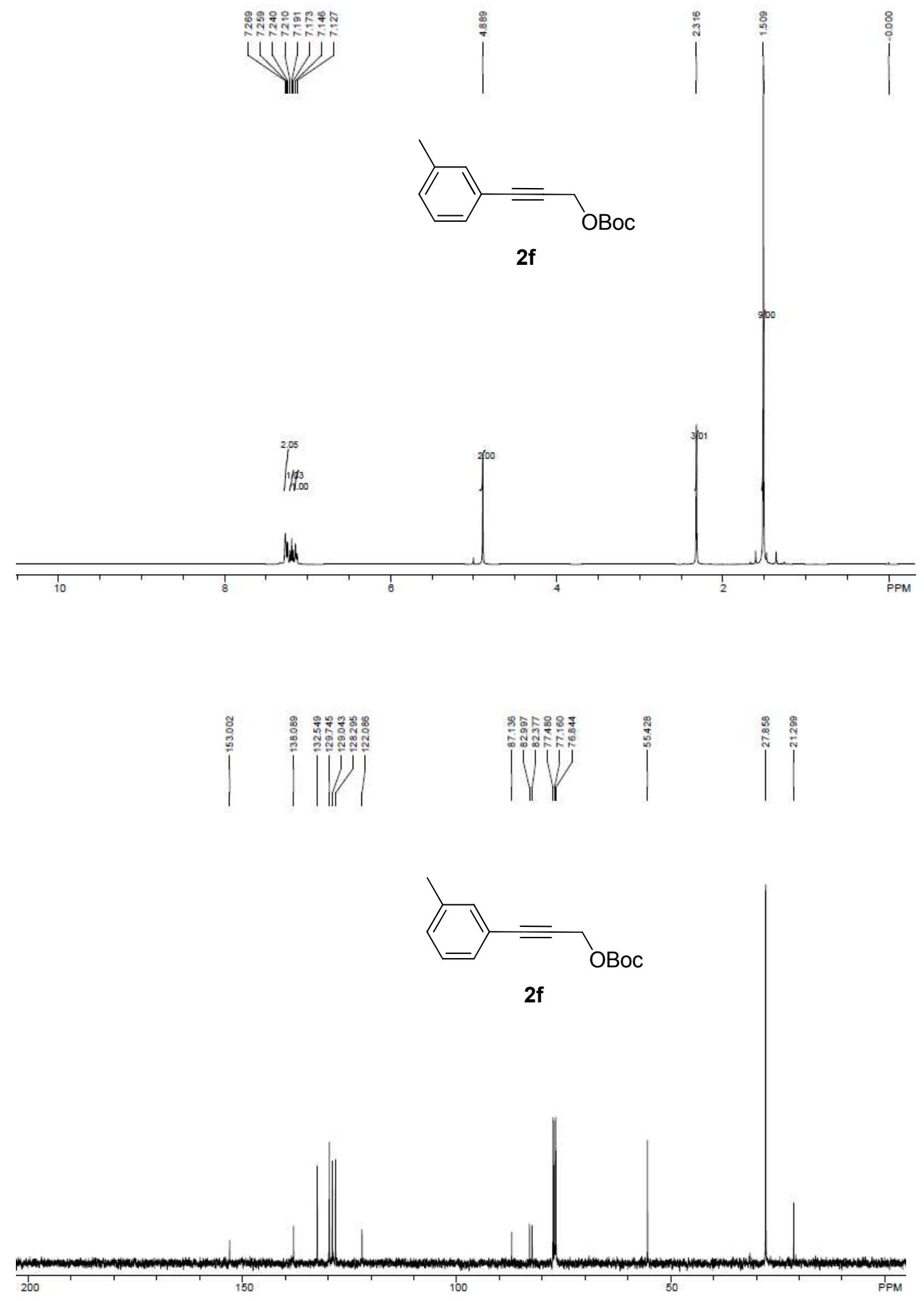




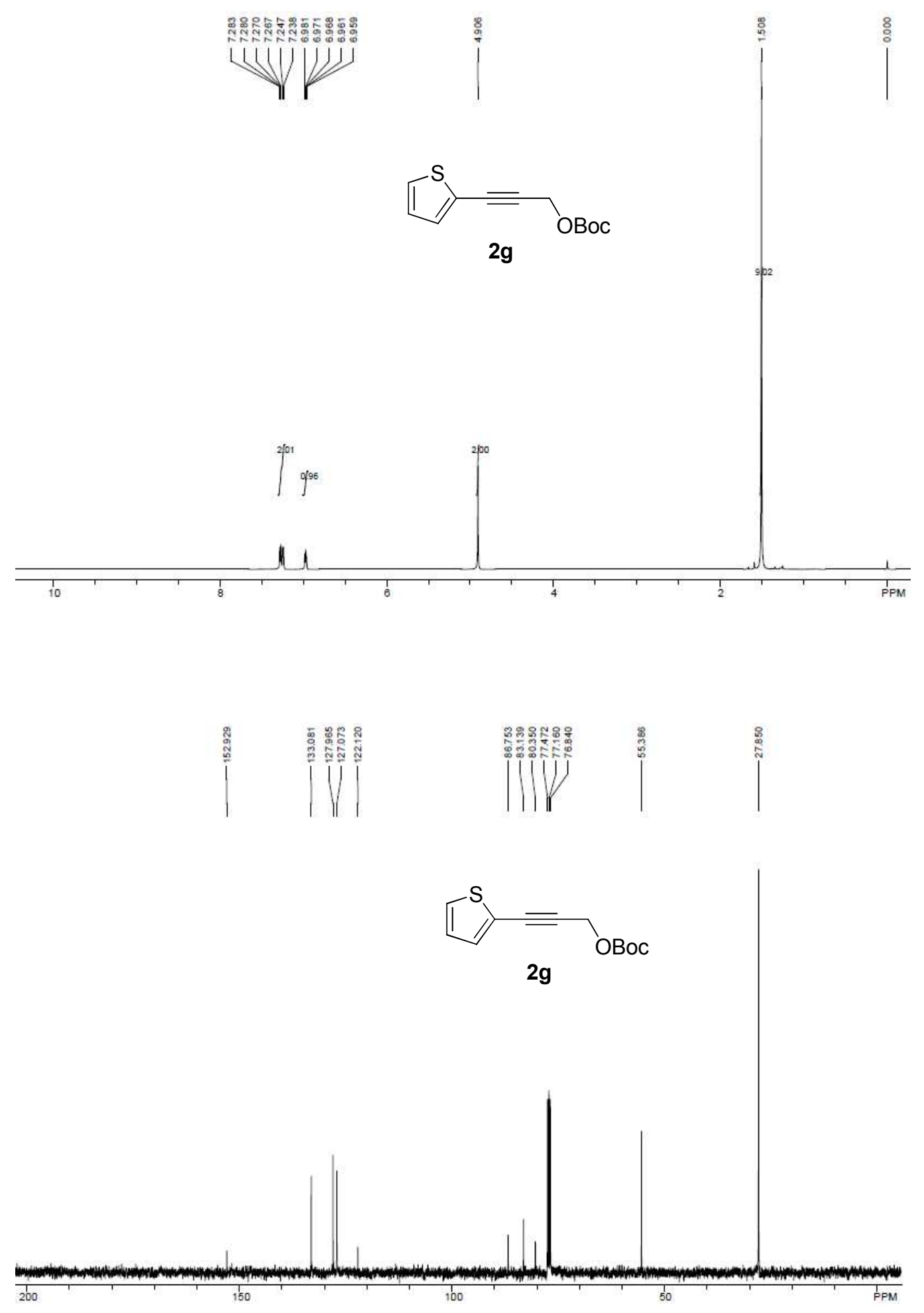



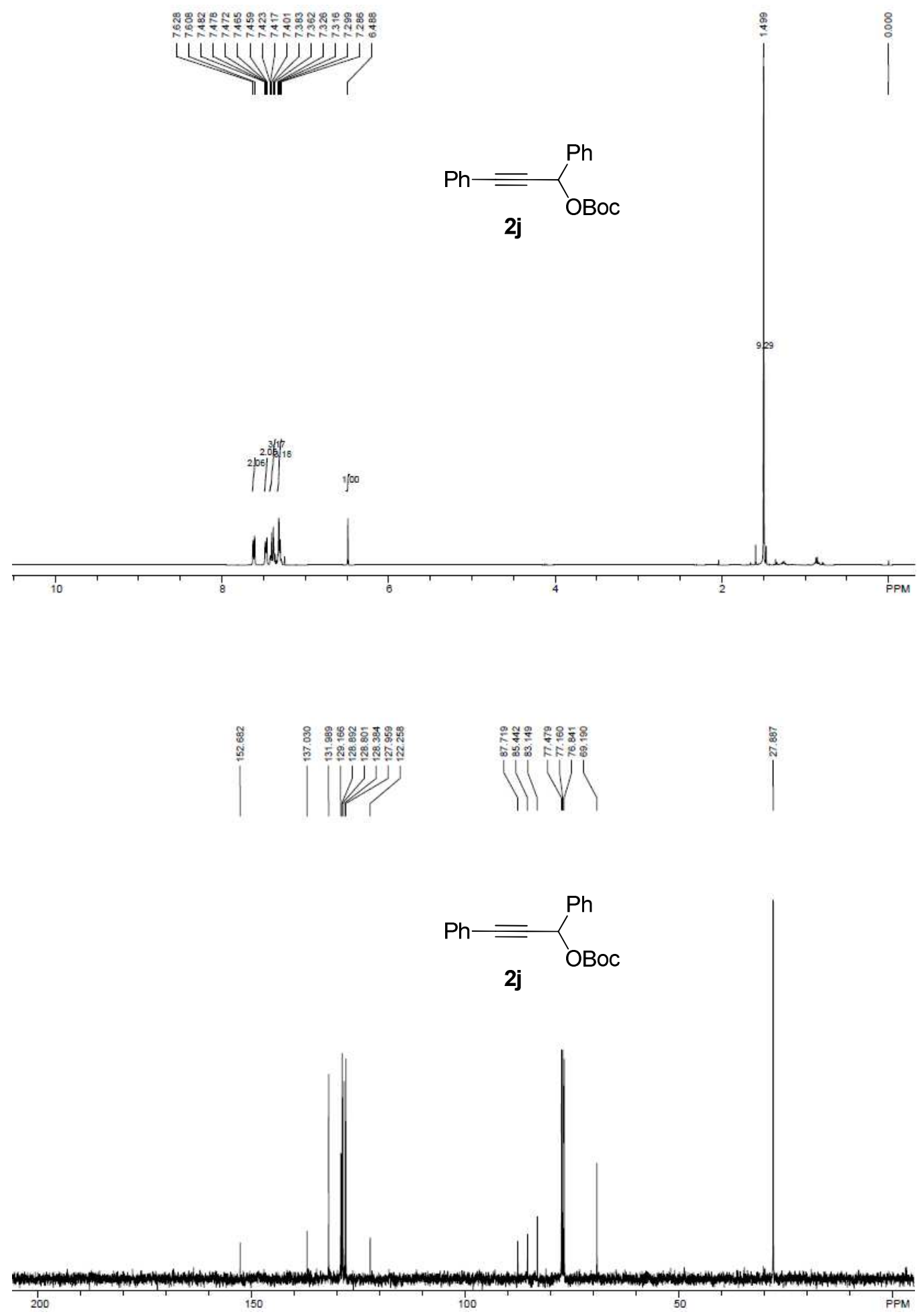

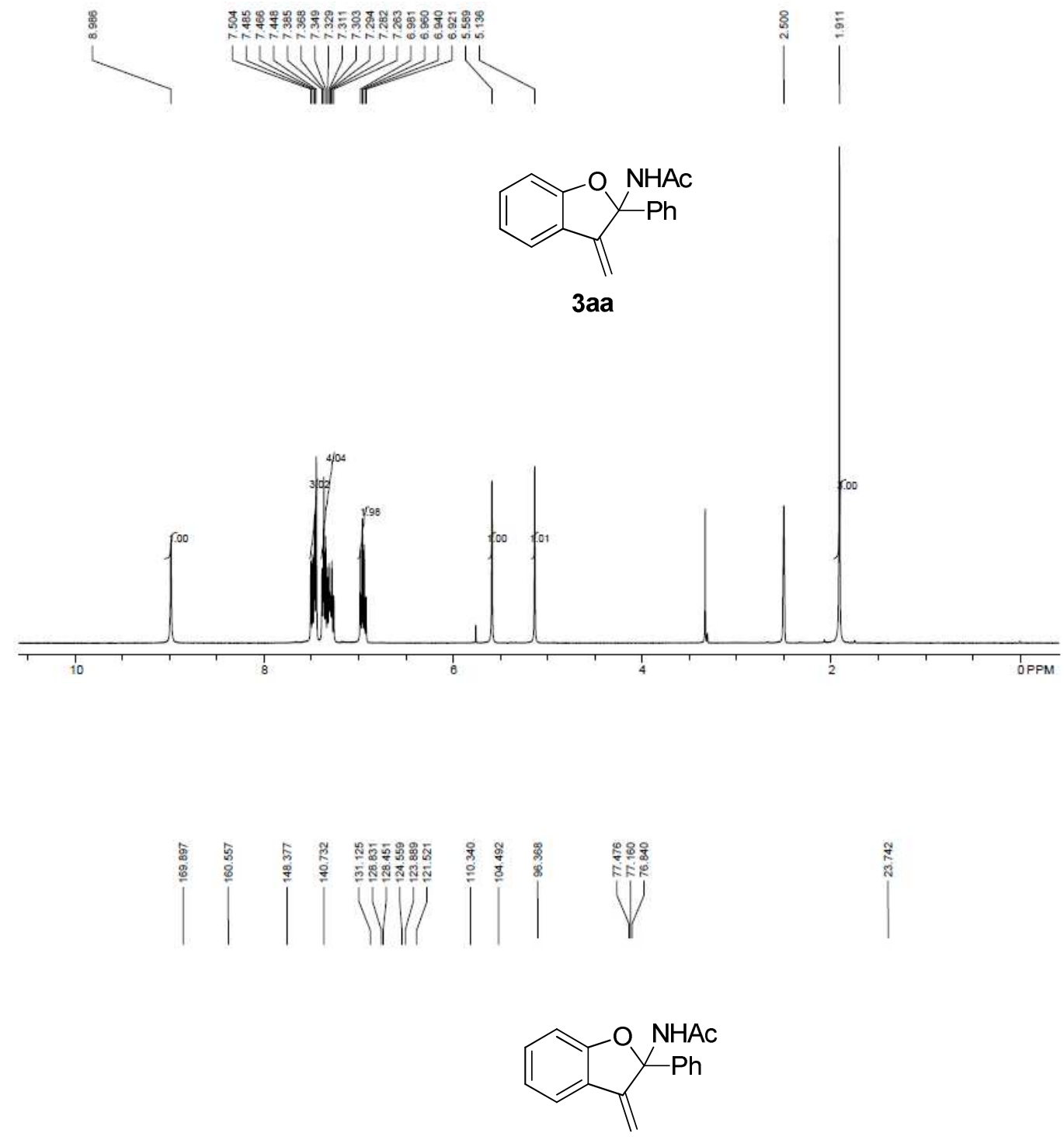

3aa

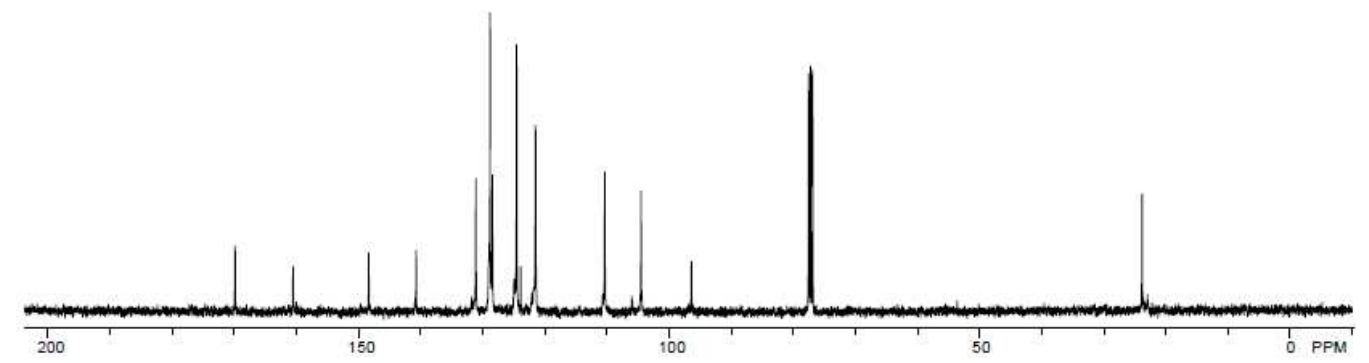



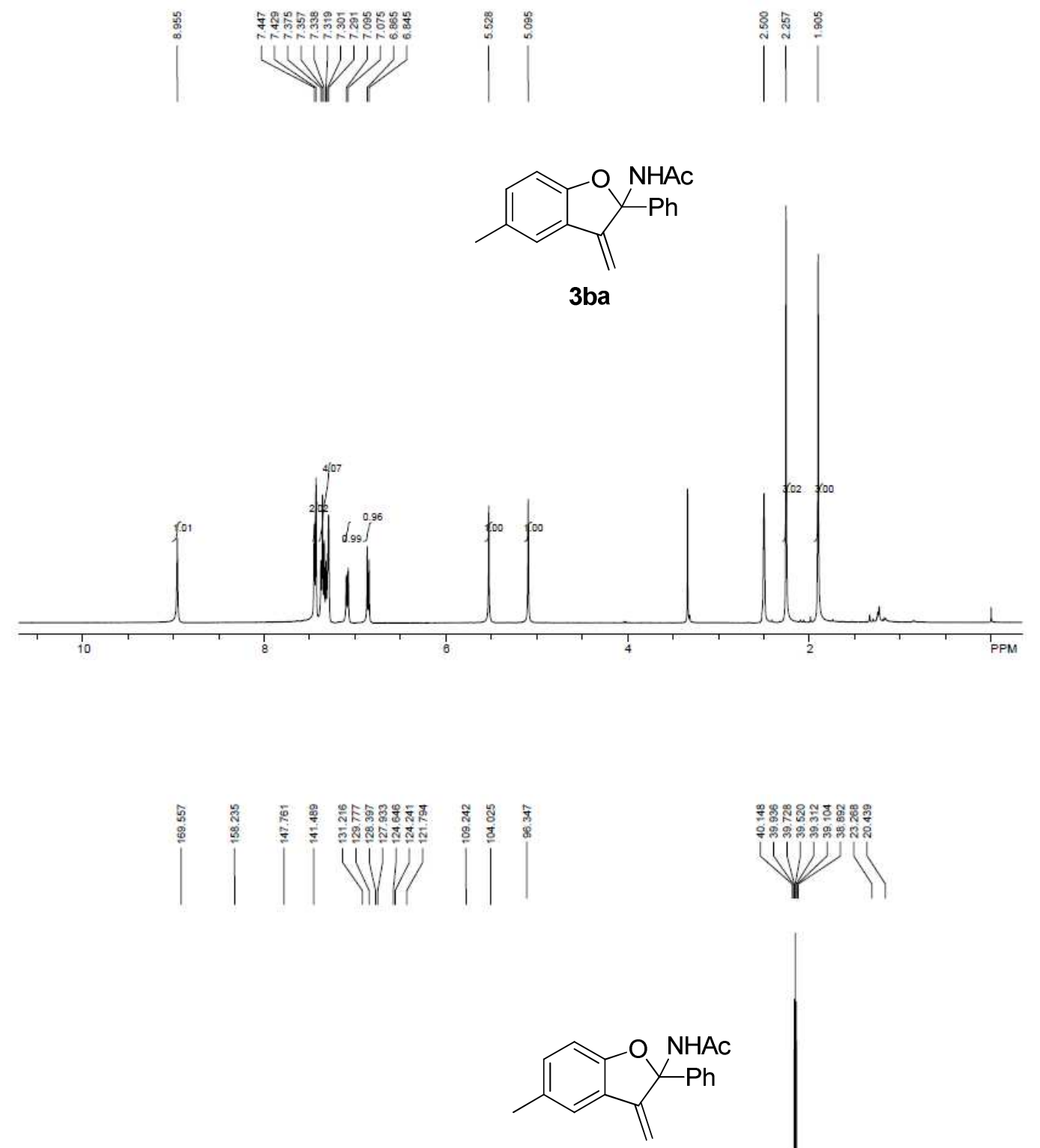

3 ba

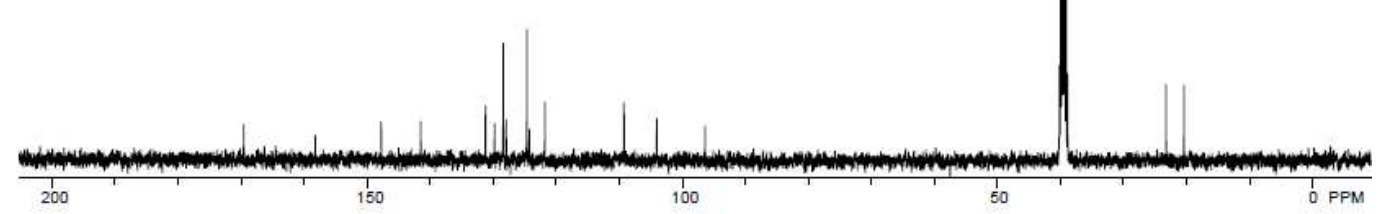



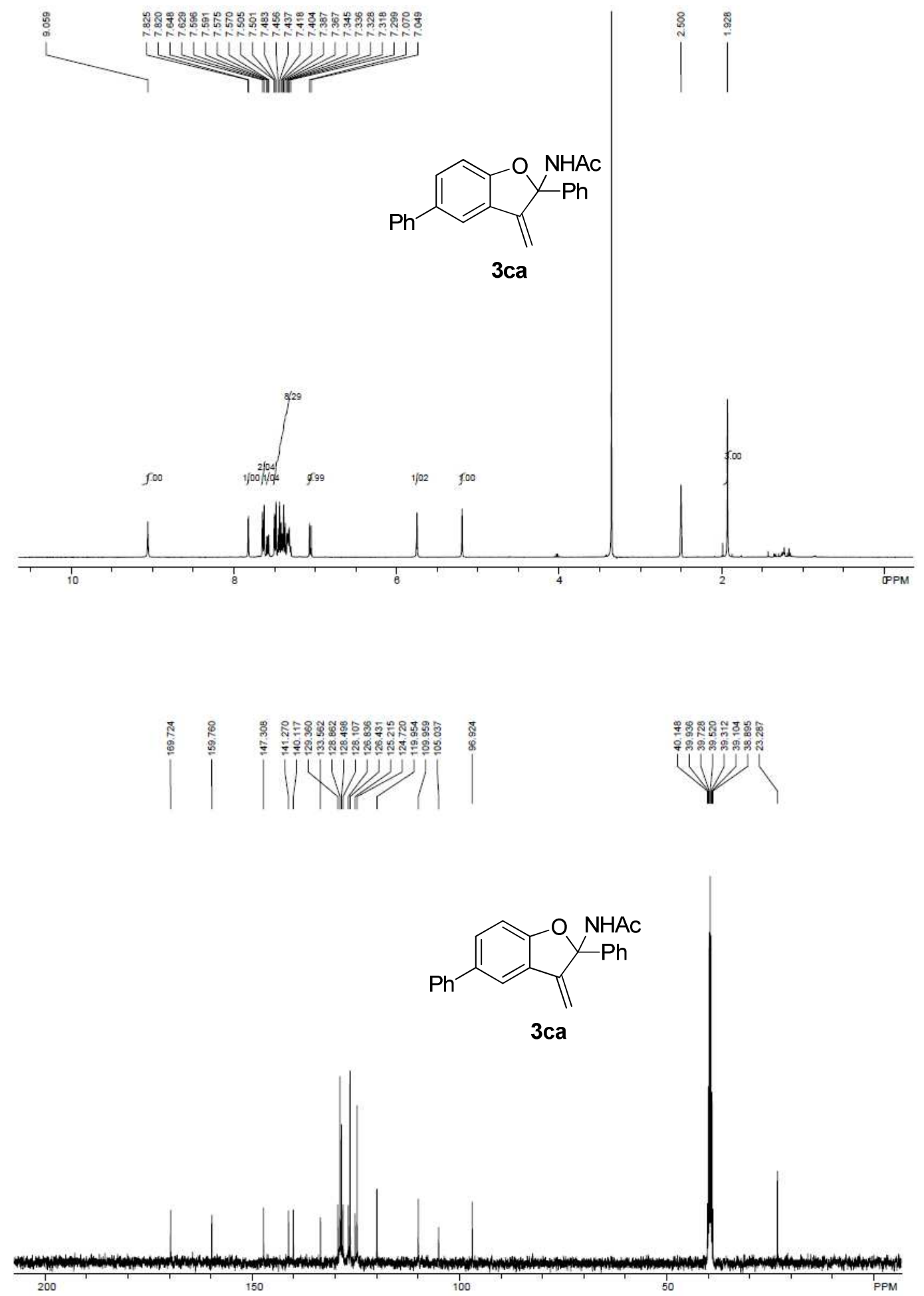

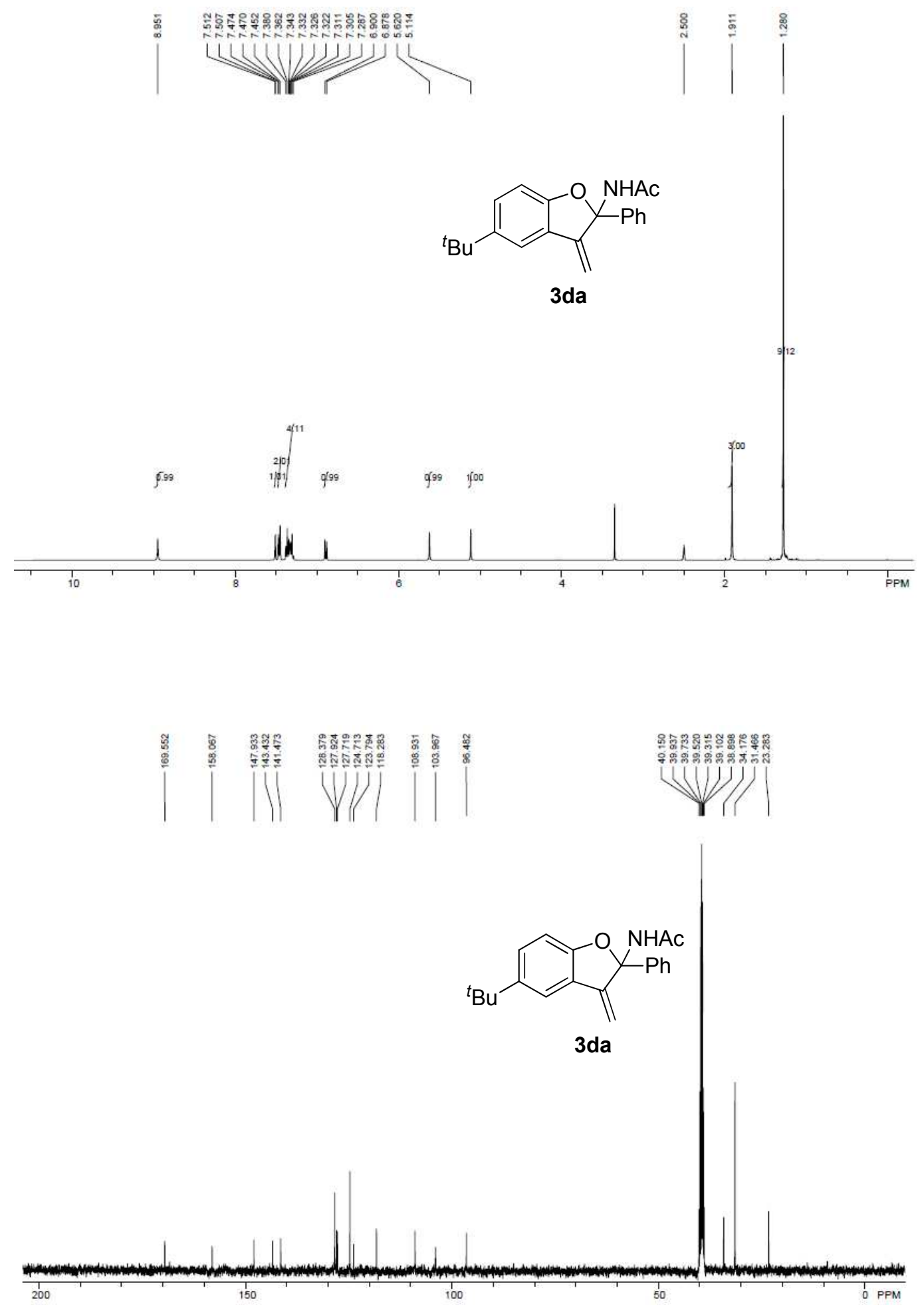

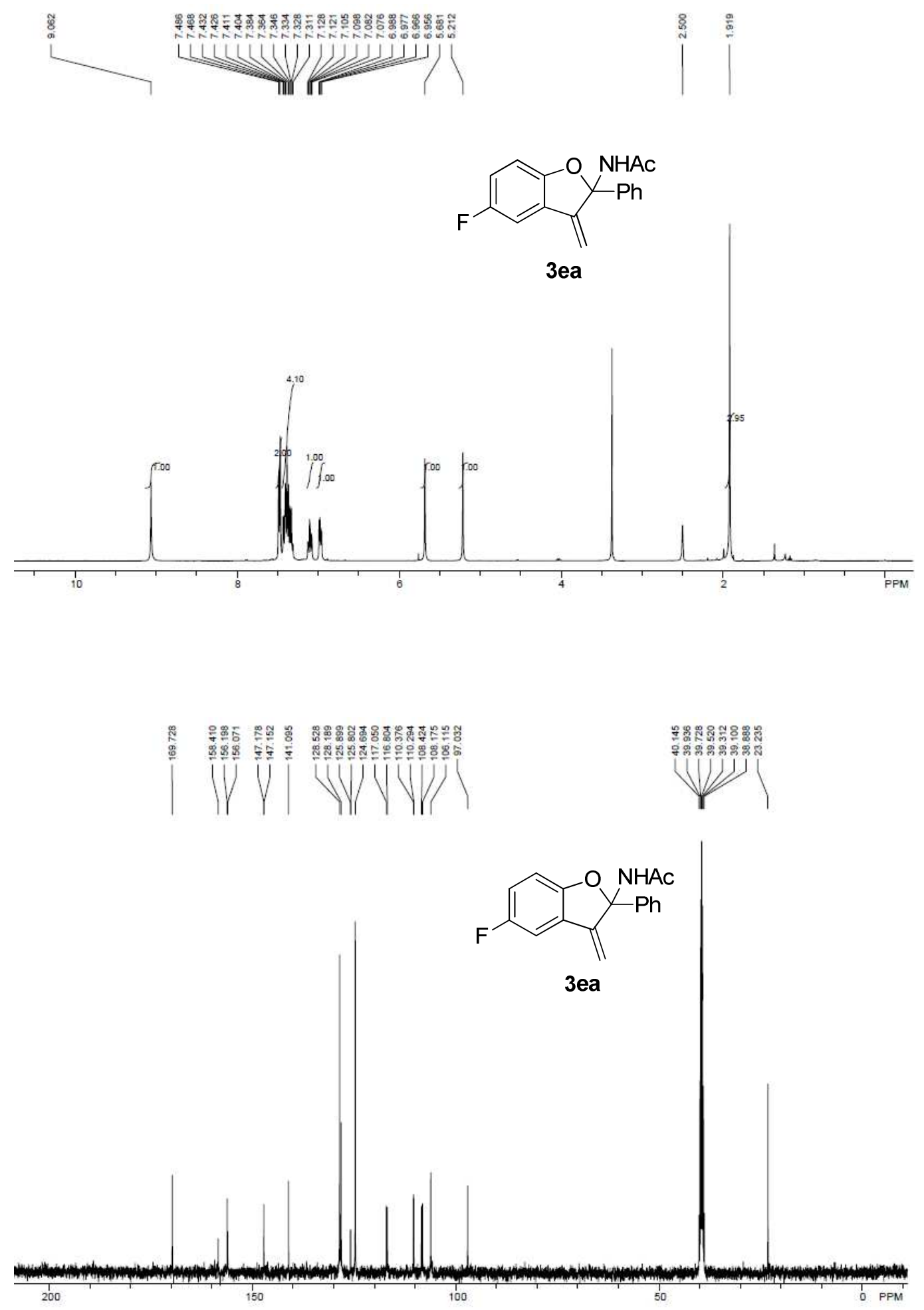

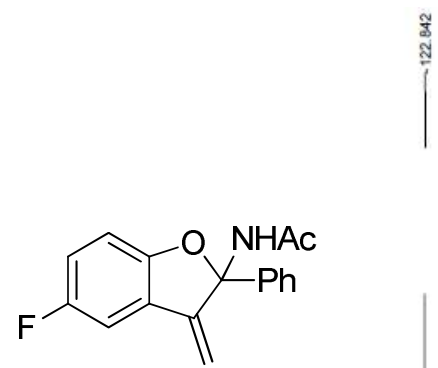

3ea

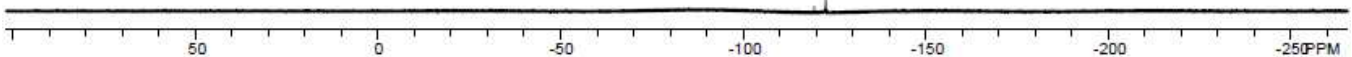



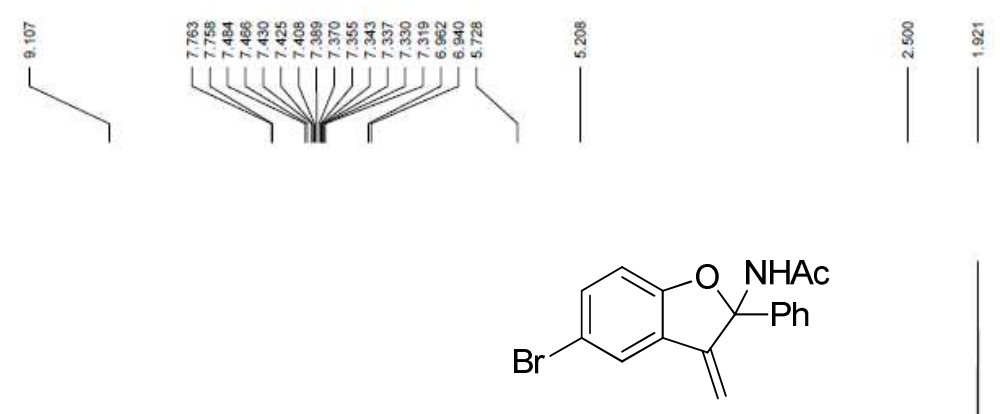

$3 f a$
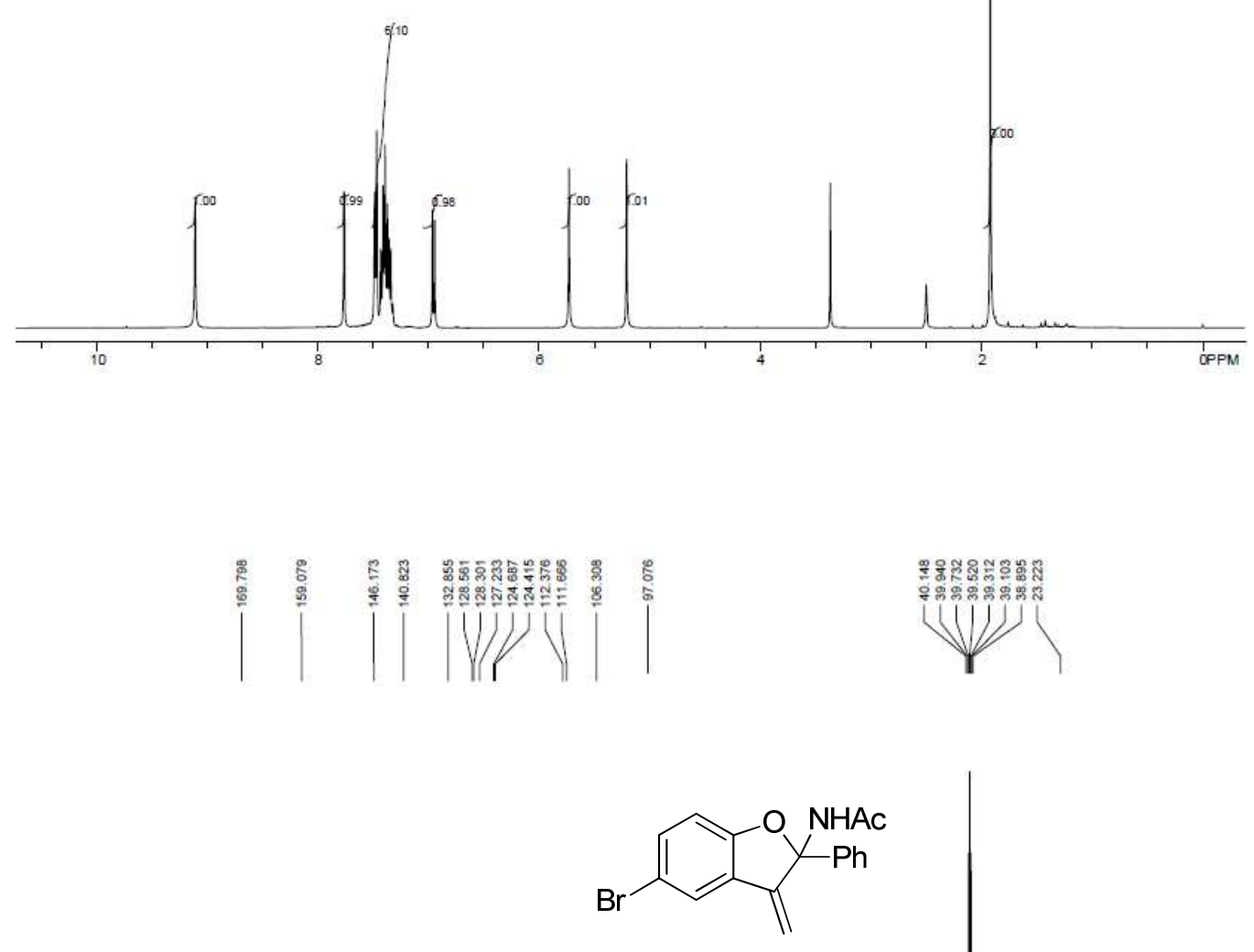

$3 f a$

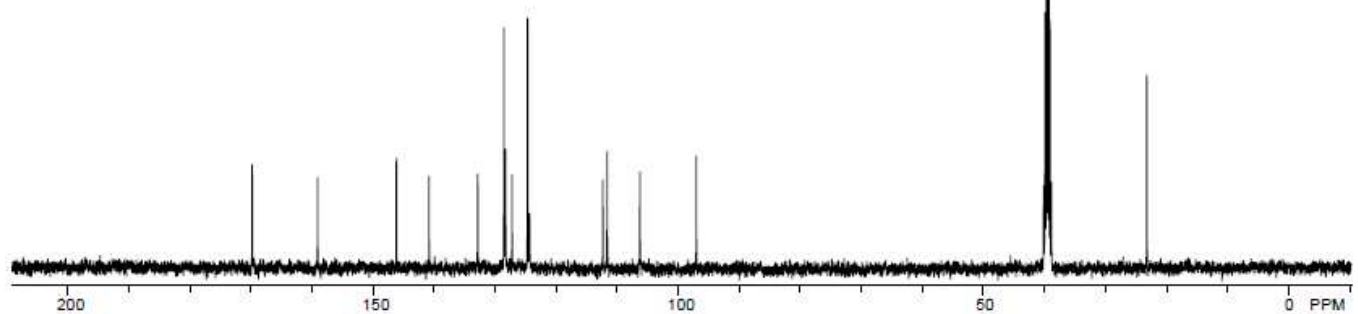




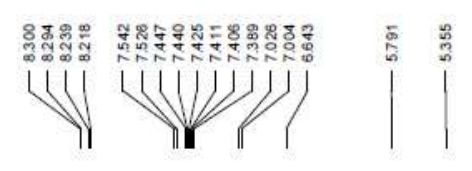<smiles>C=C1c2cc([N+](=O)[O-])ccc2OC1(NC(C)=O)c1ccccc1</smiles>

3ga
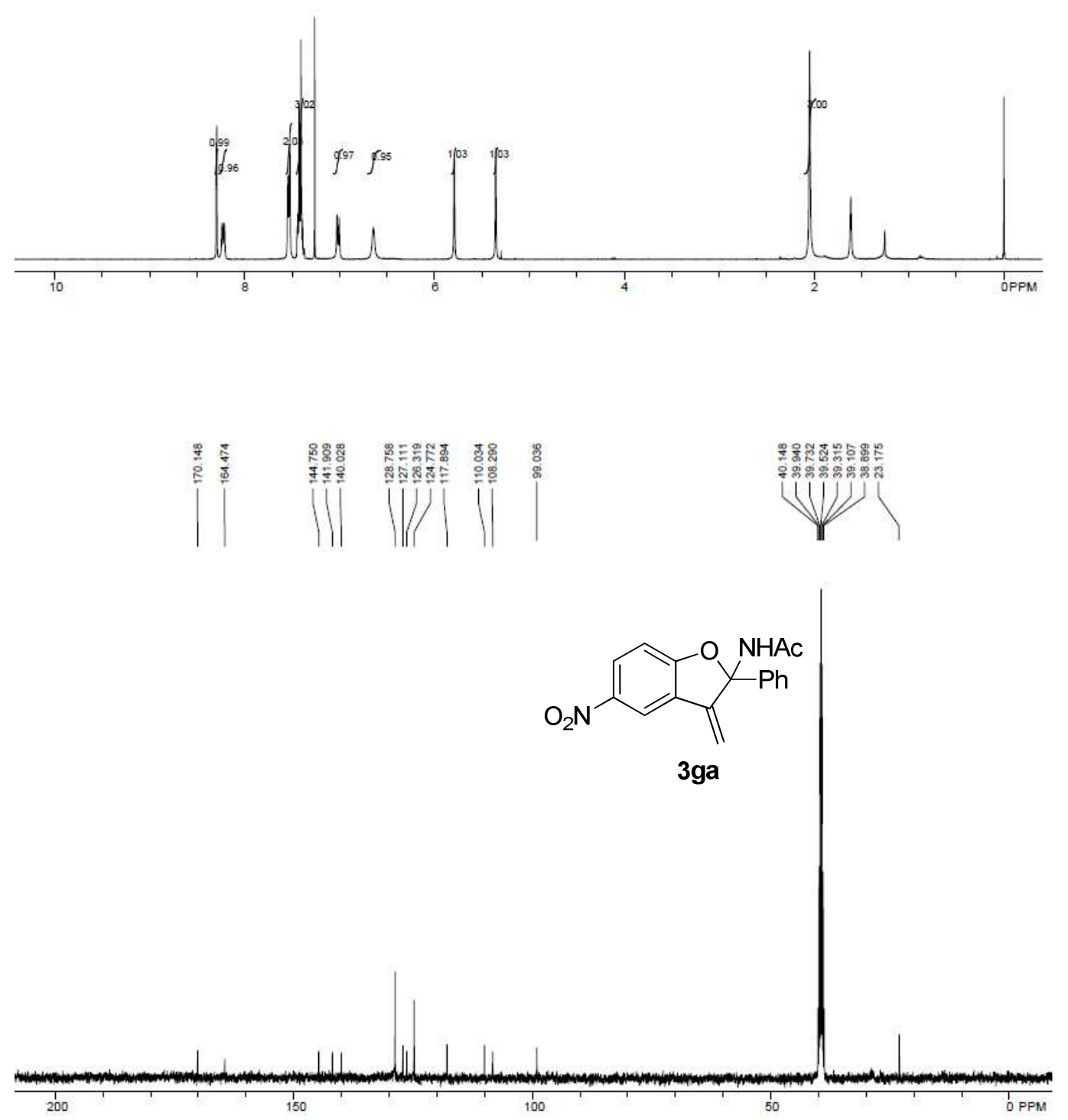

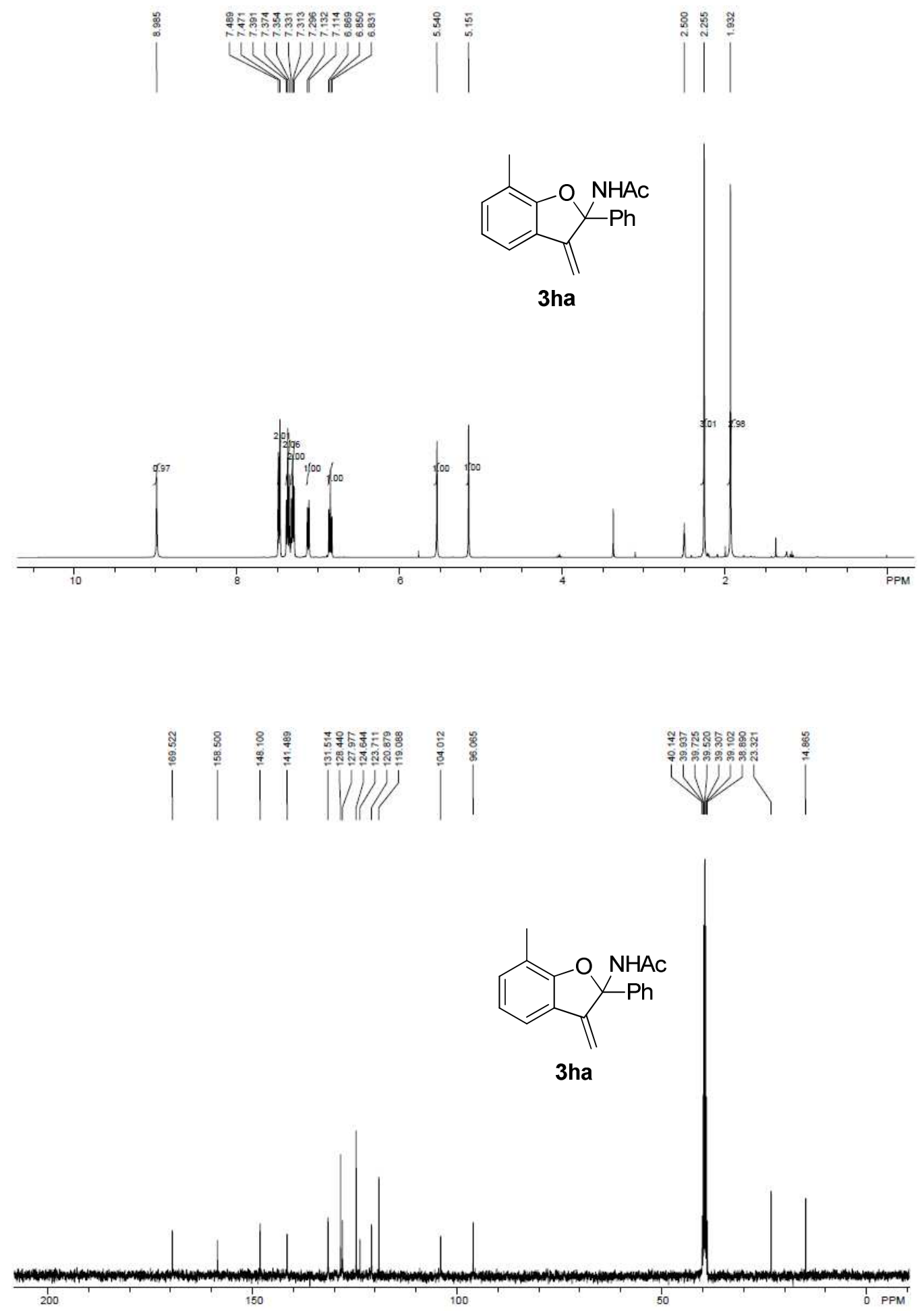


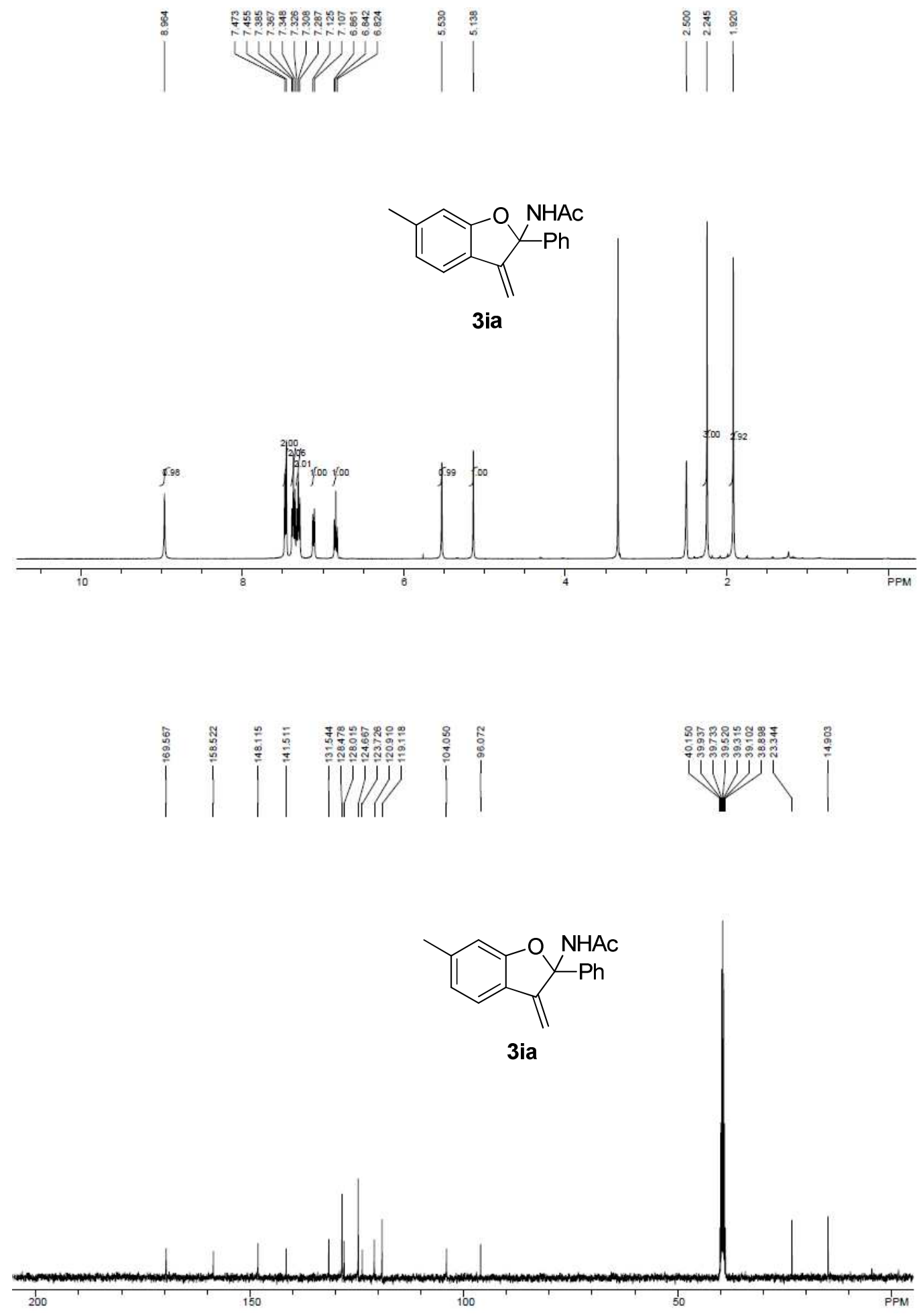



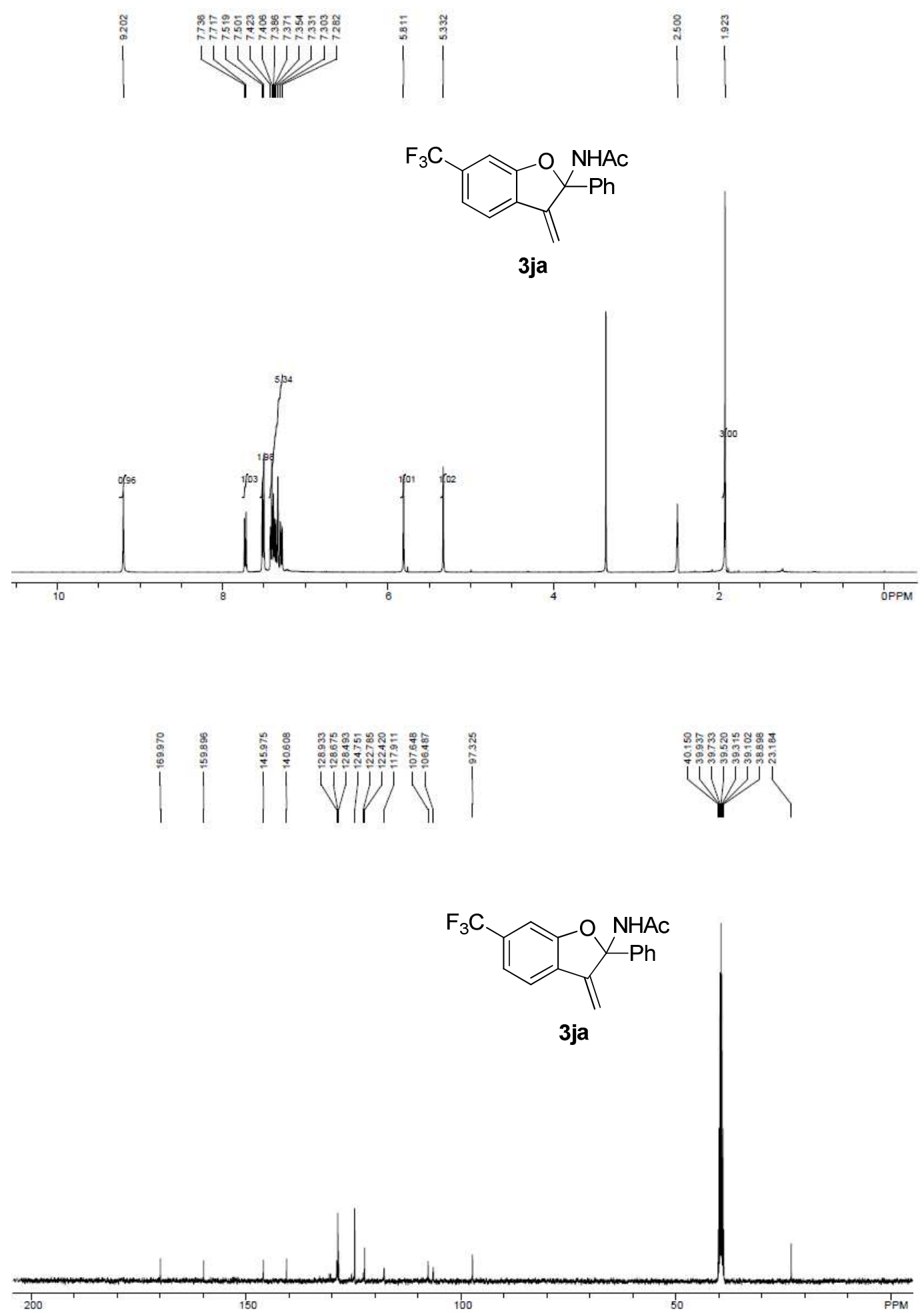


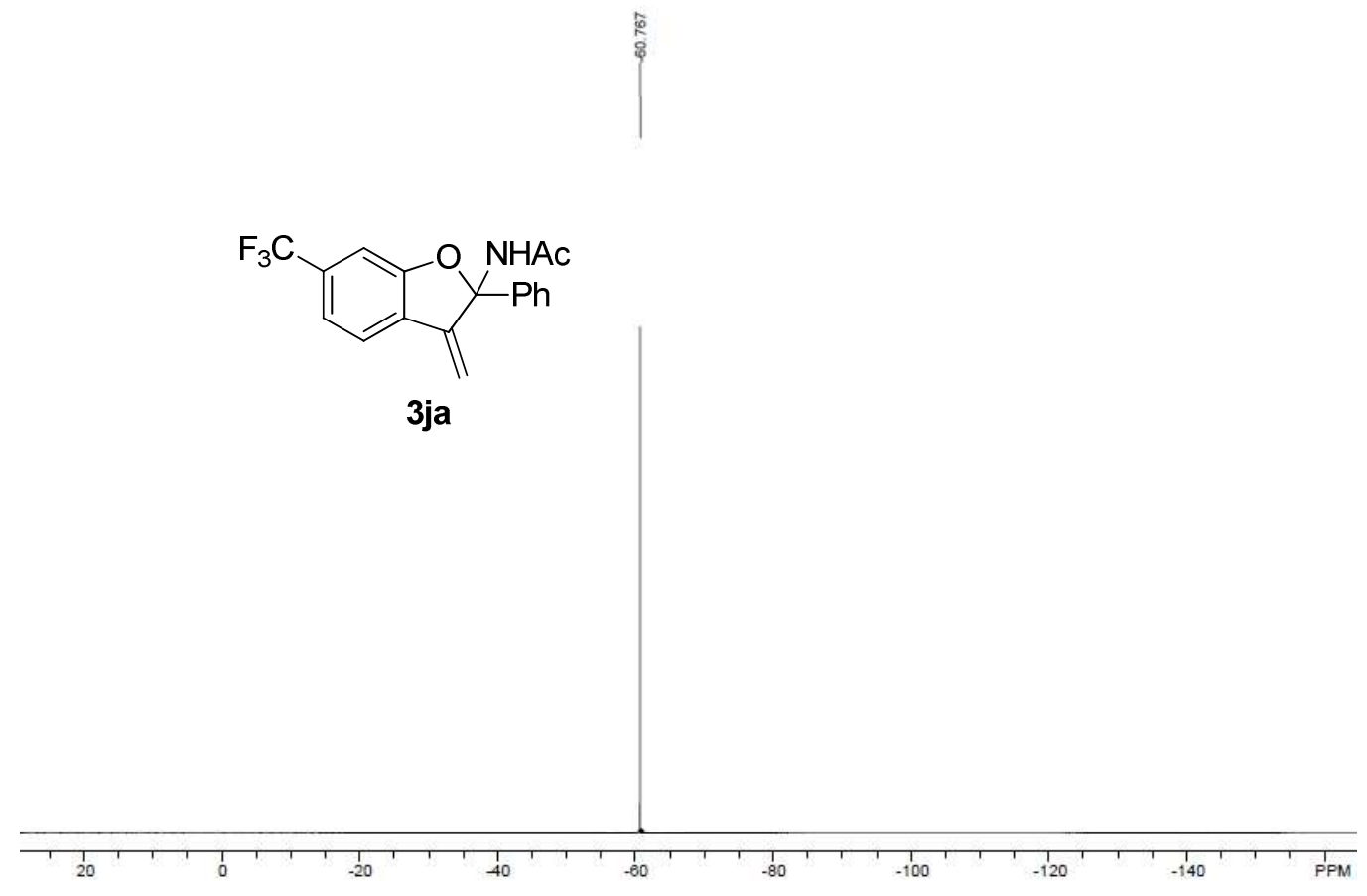



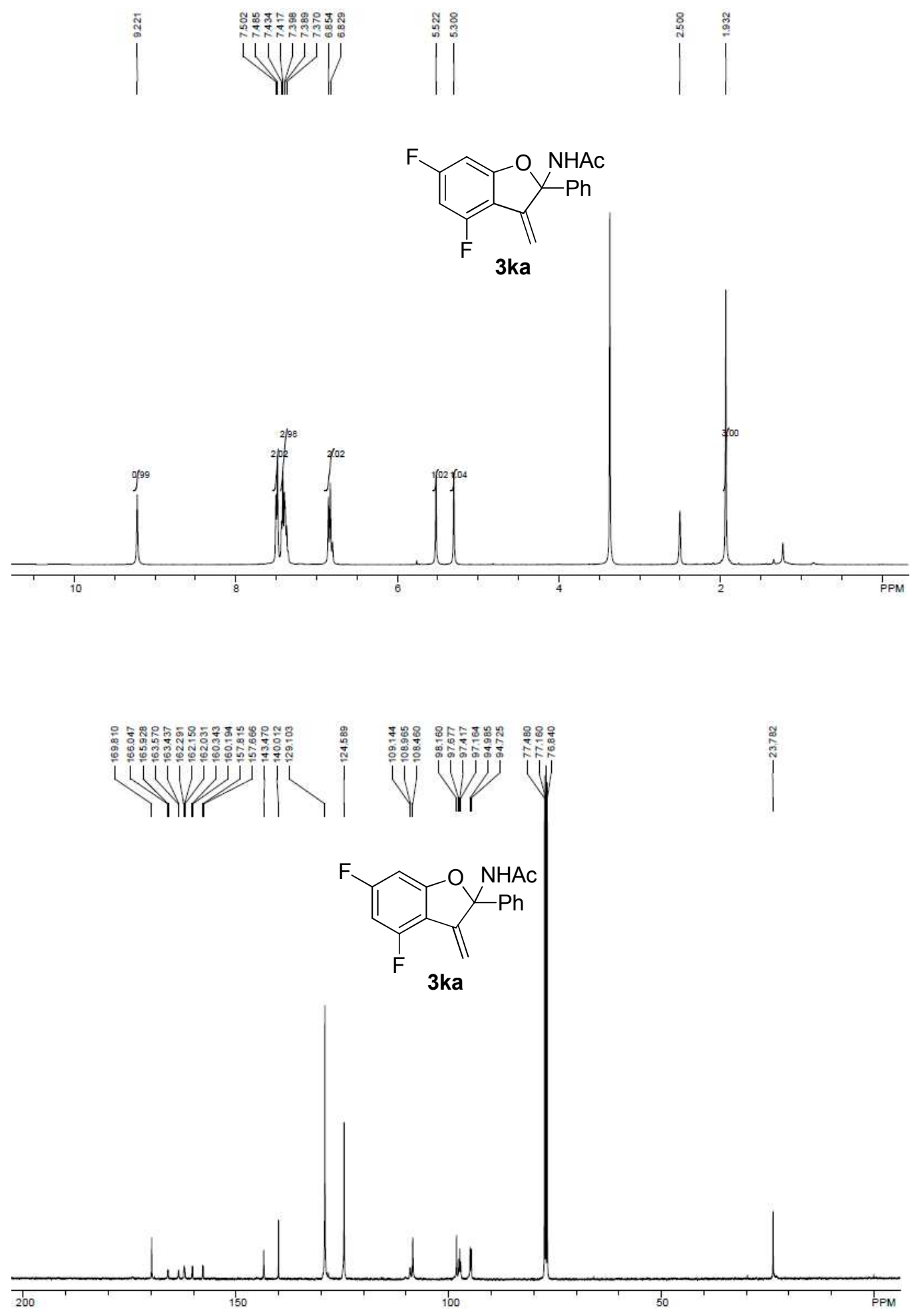

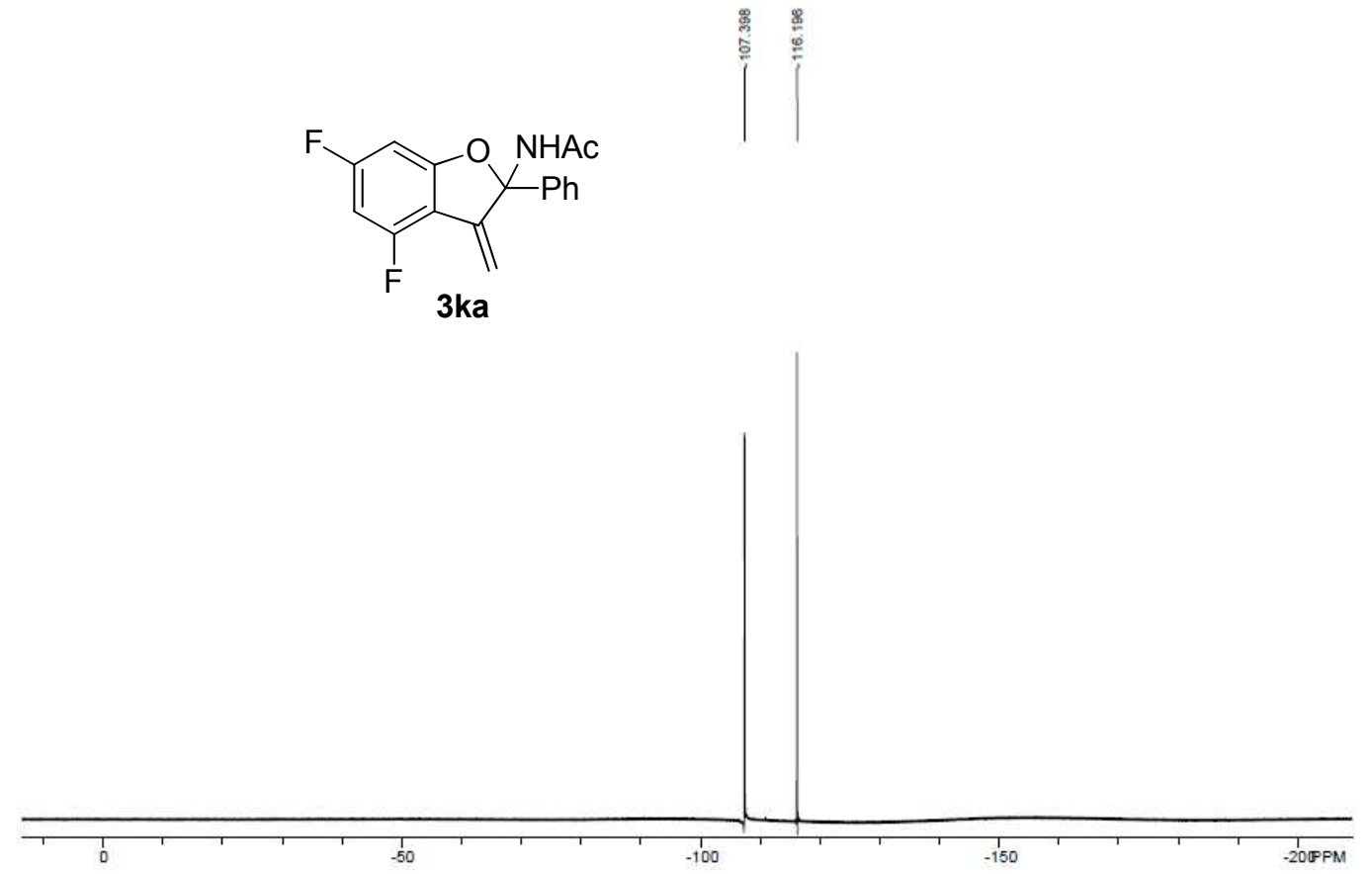


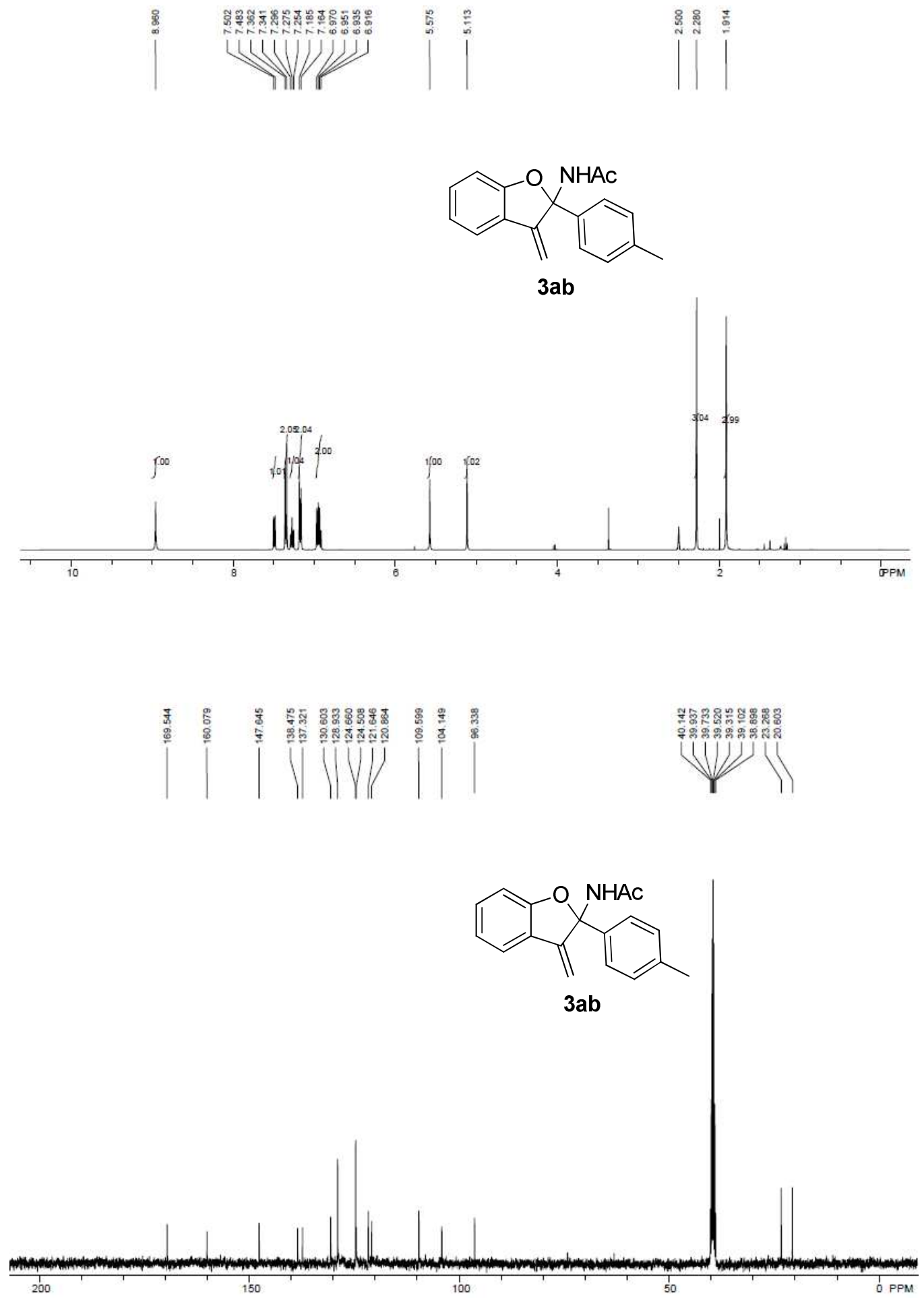



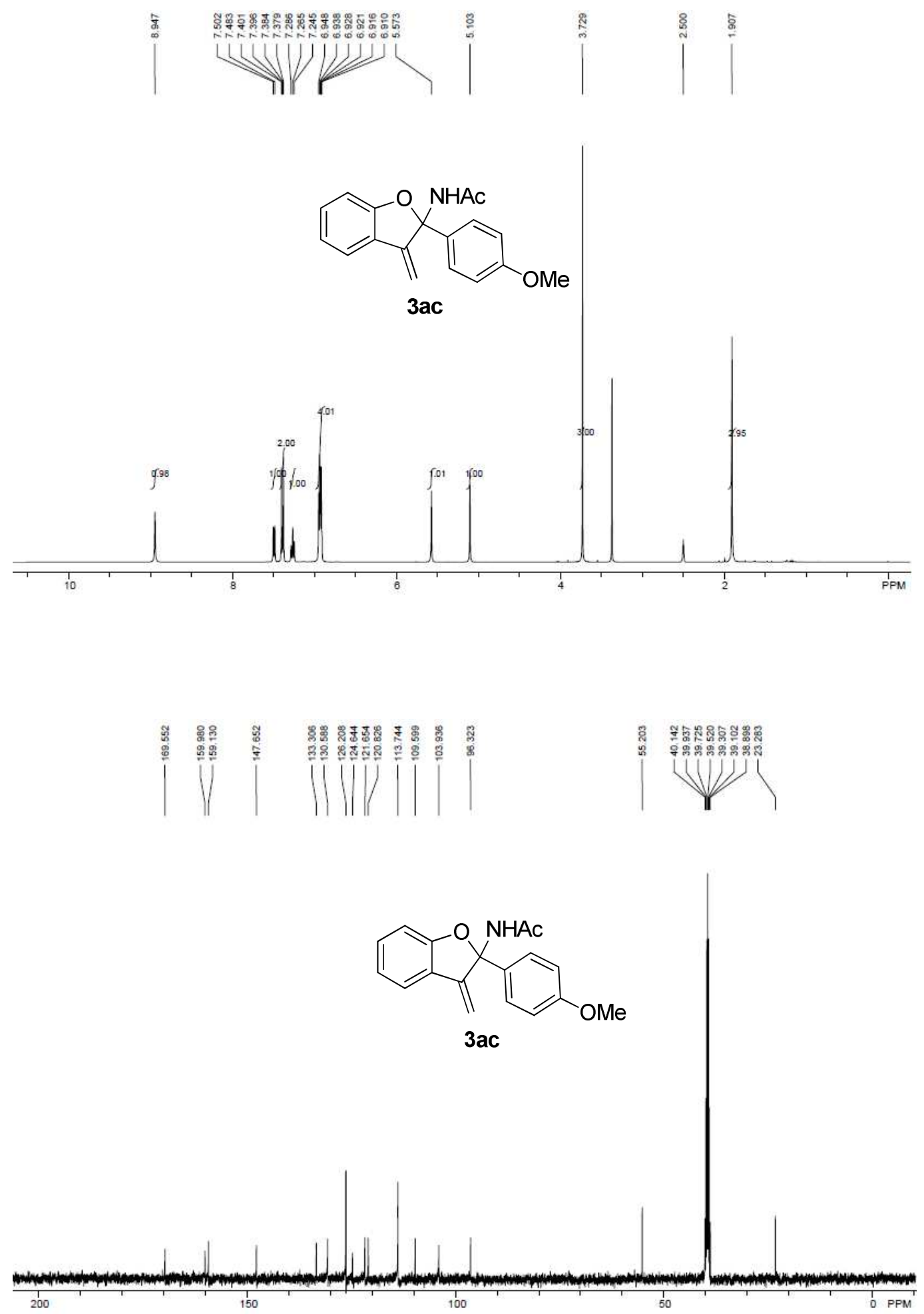

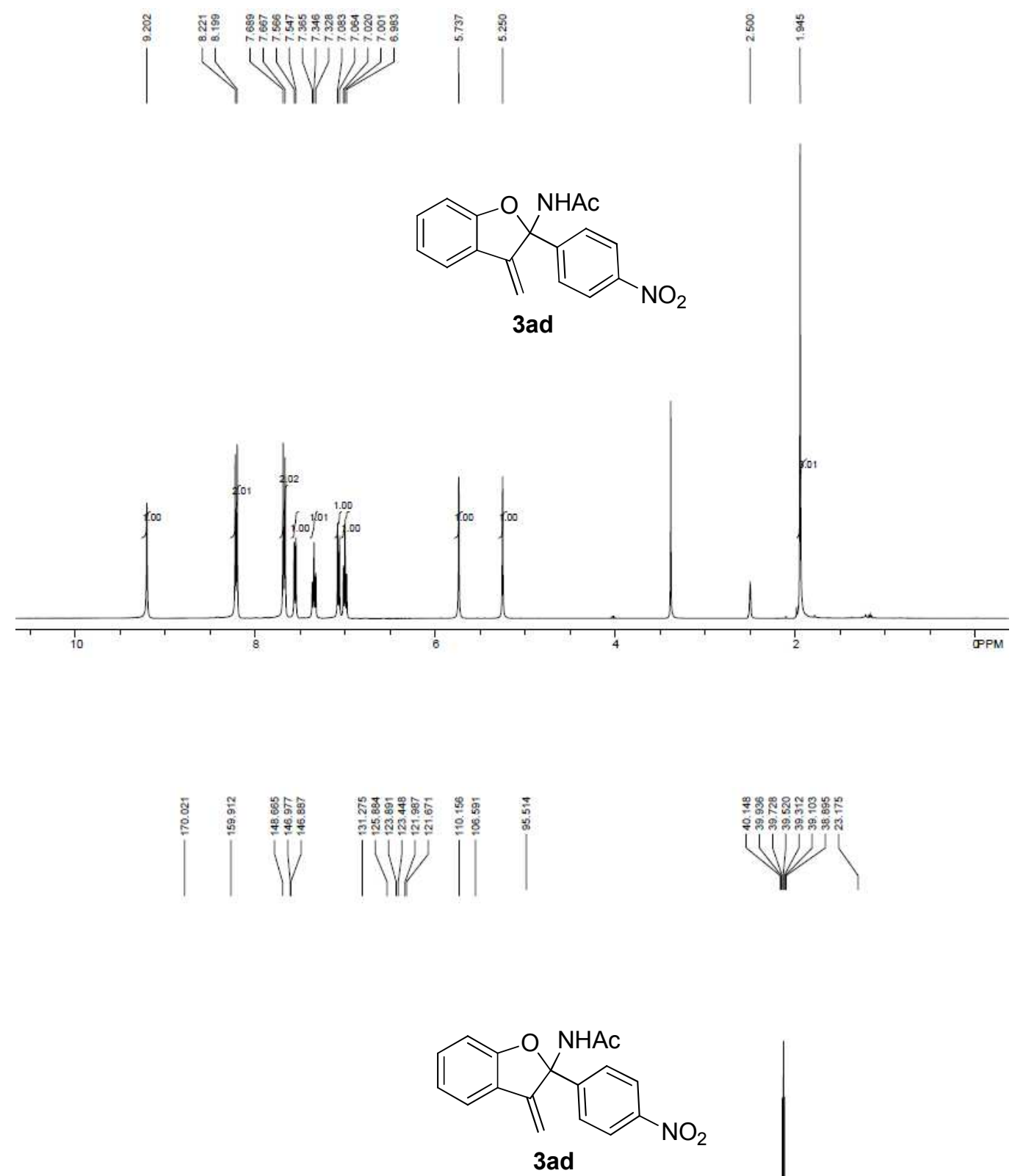

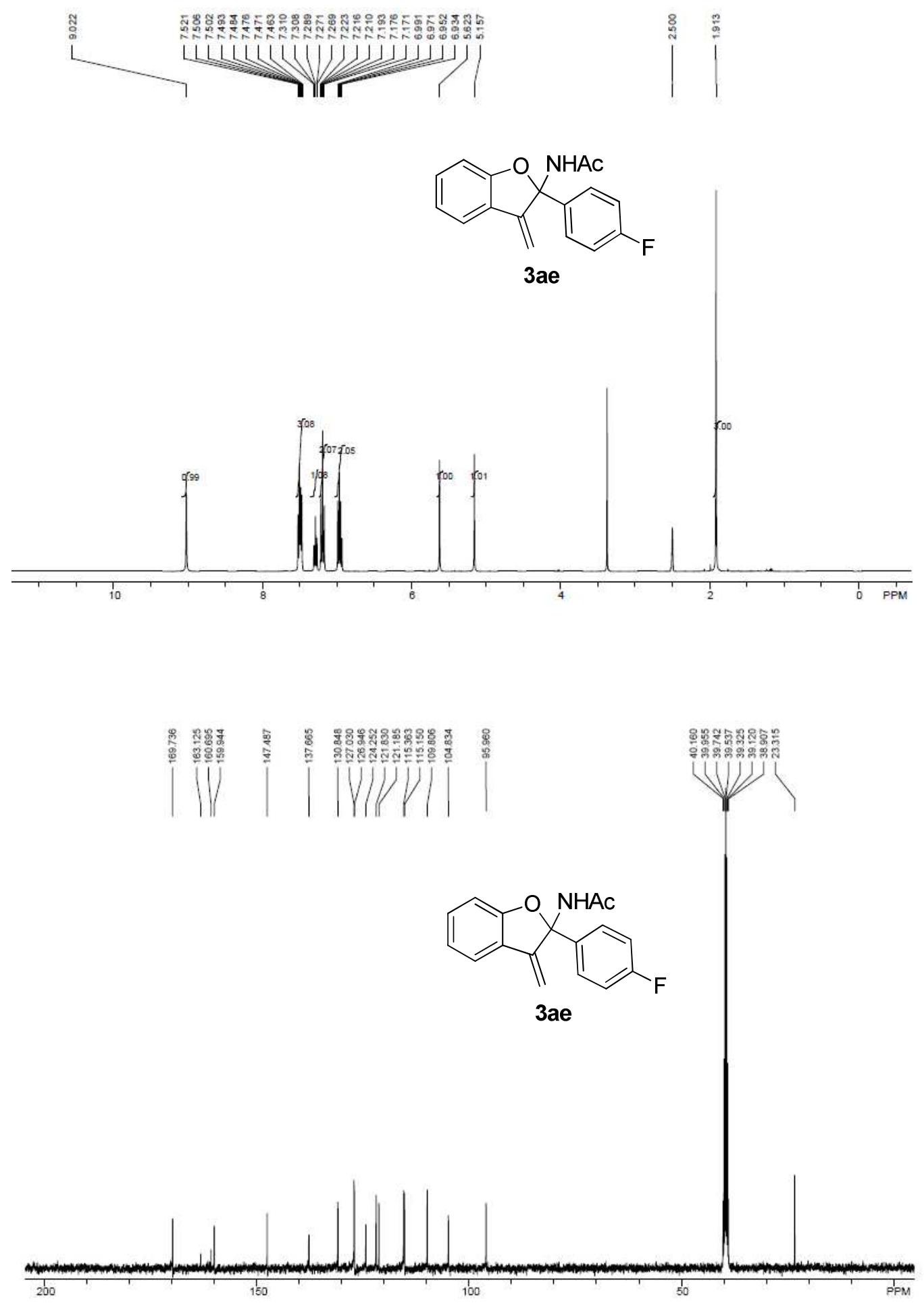


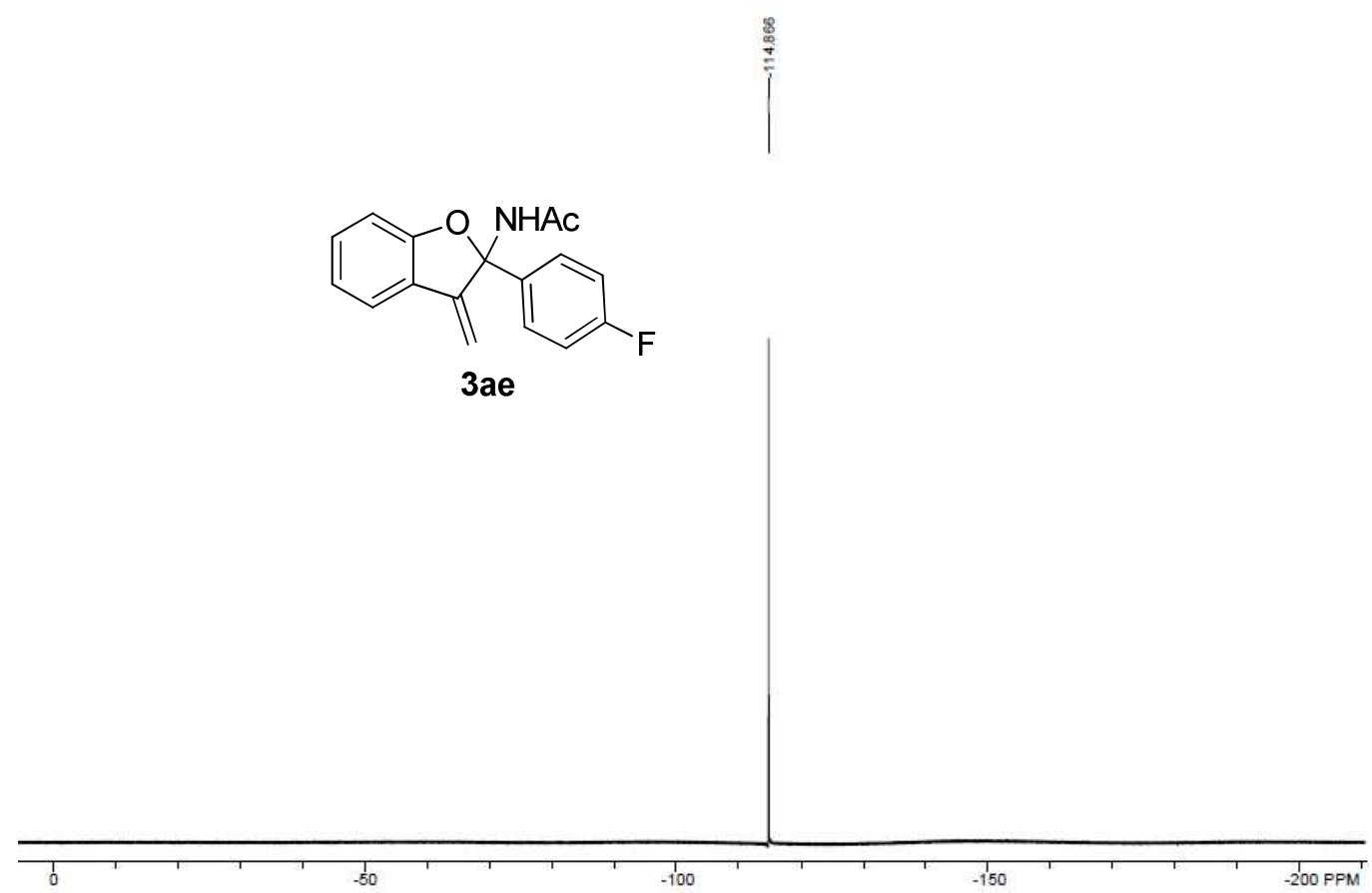



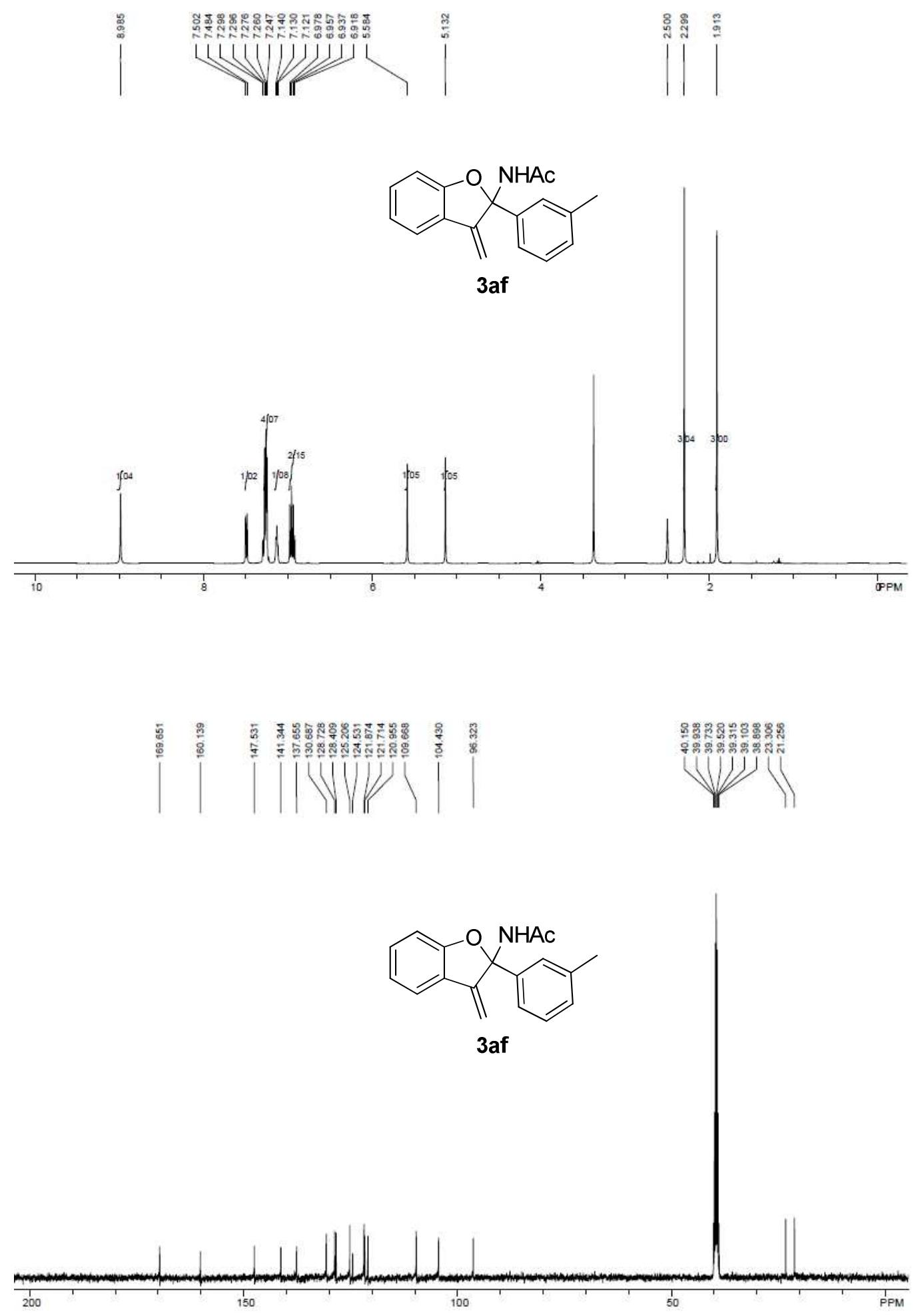


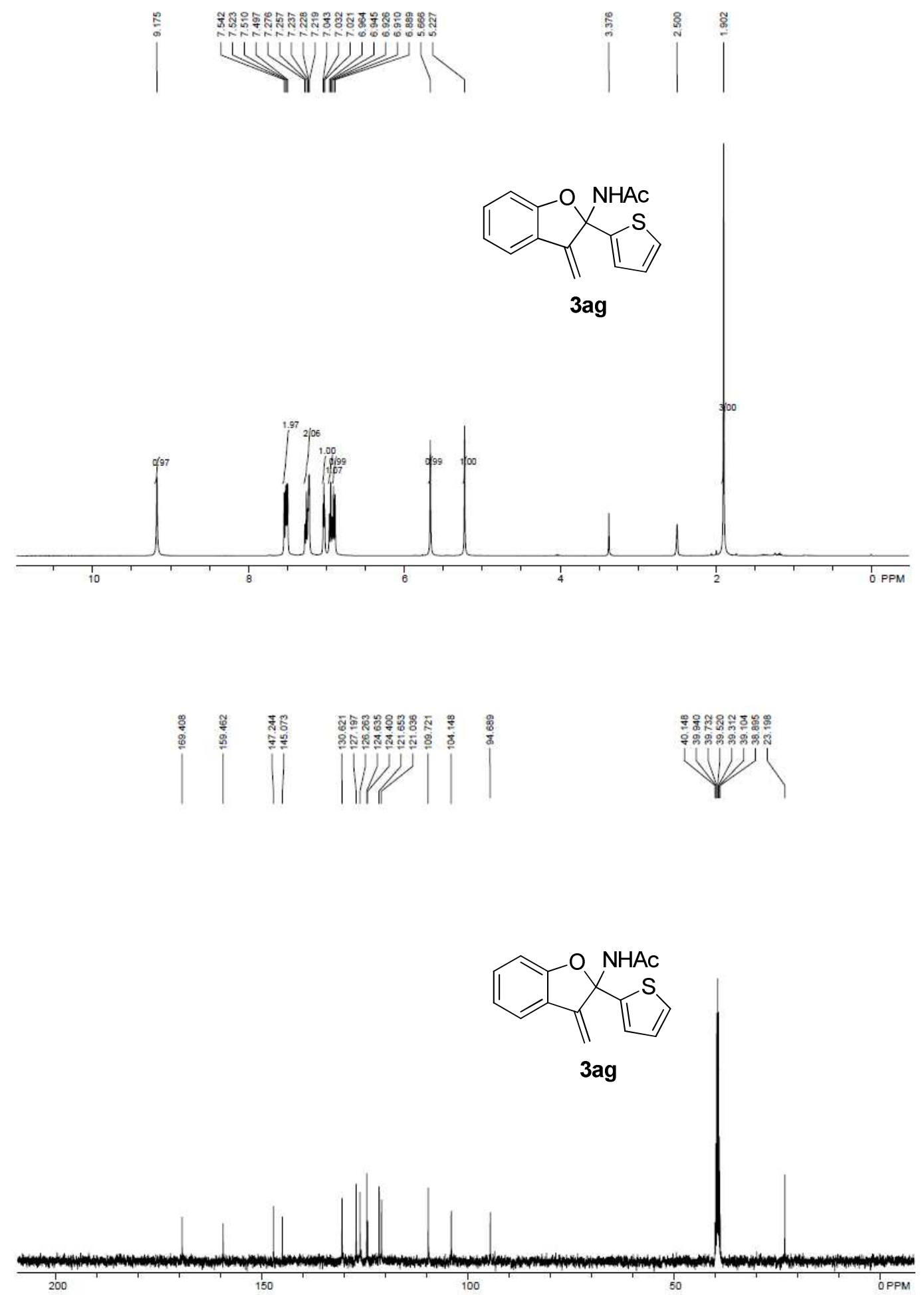



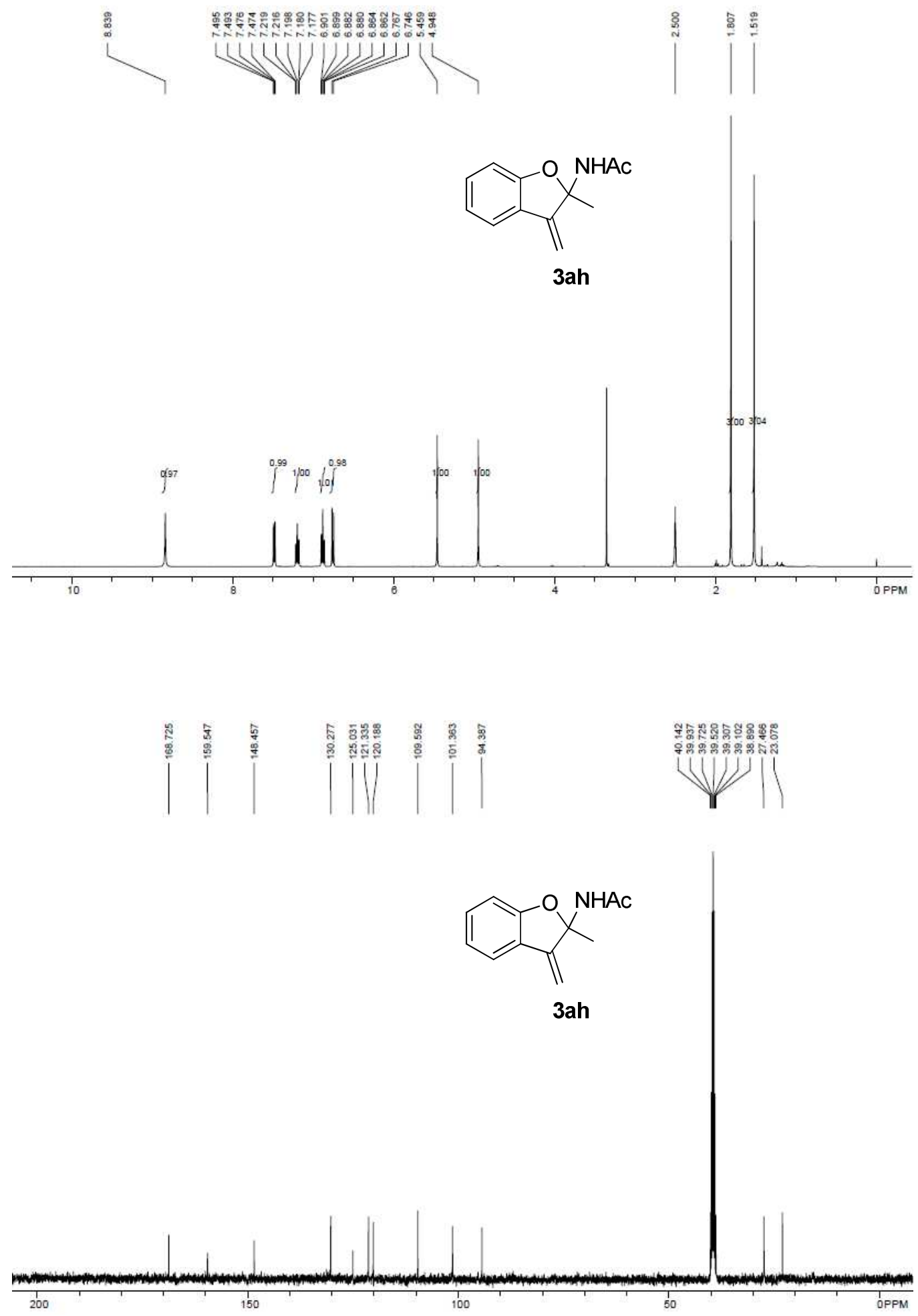

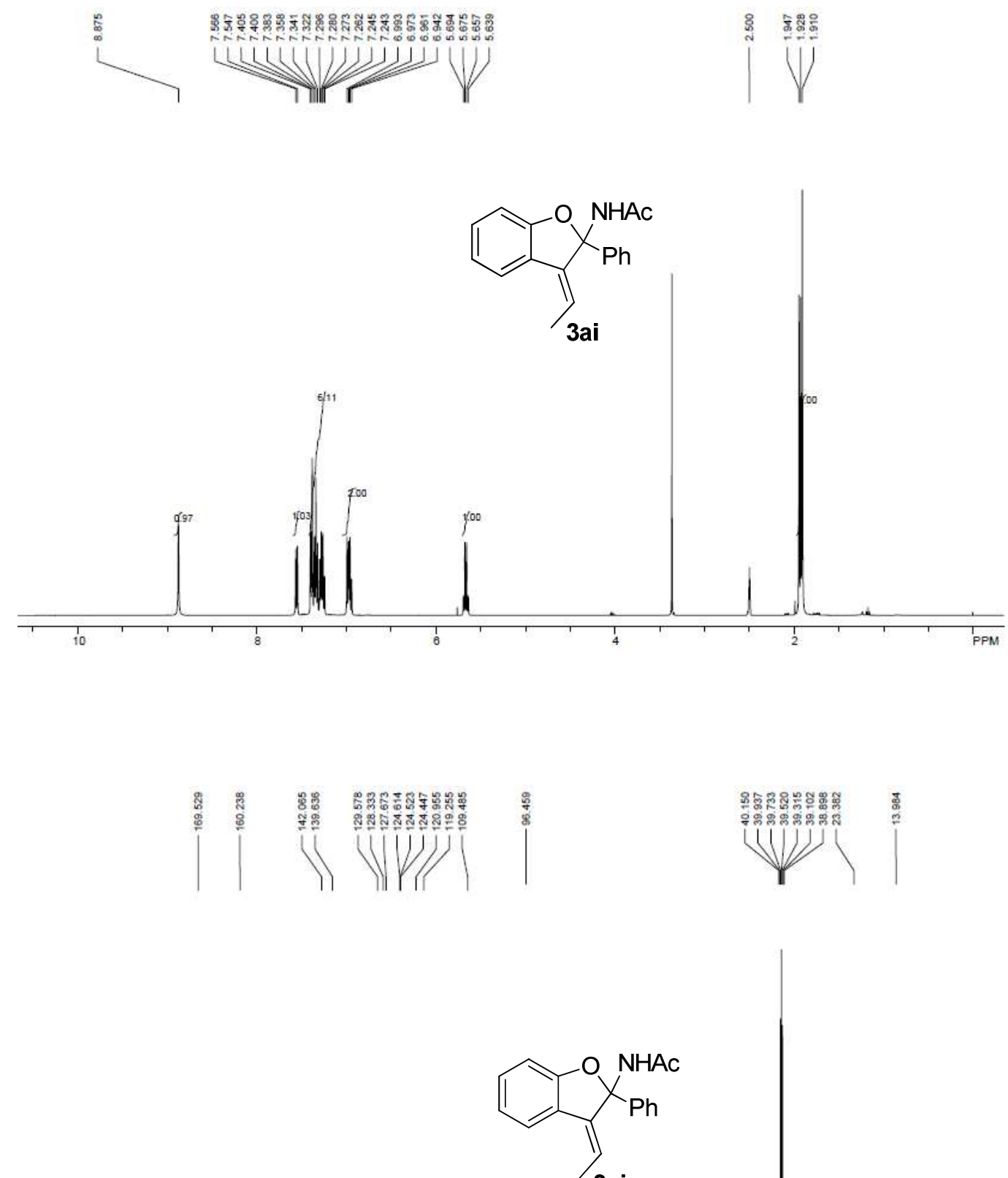

3ai

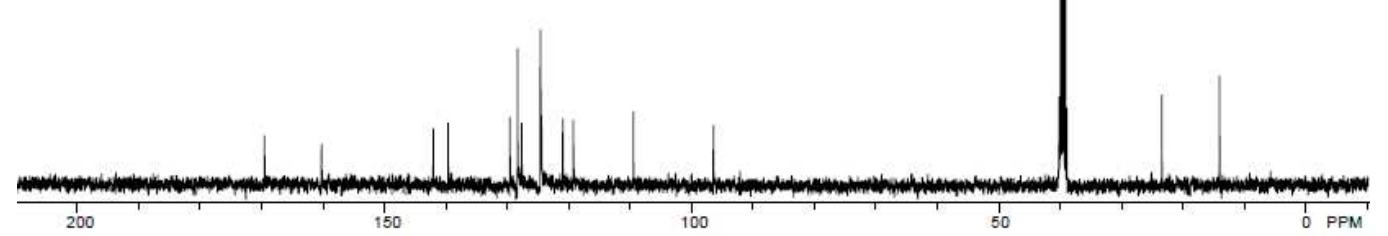




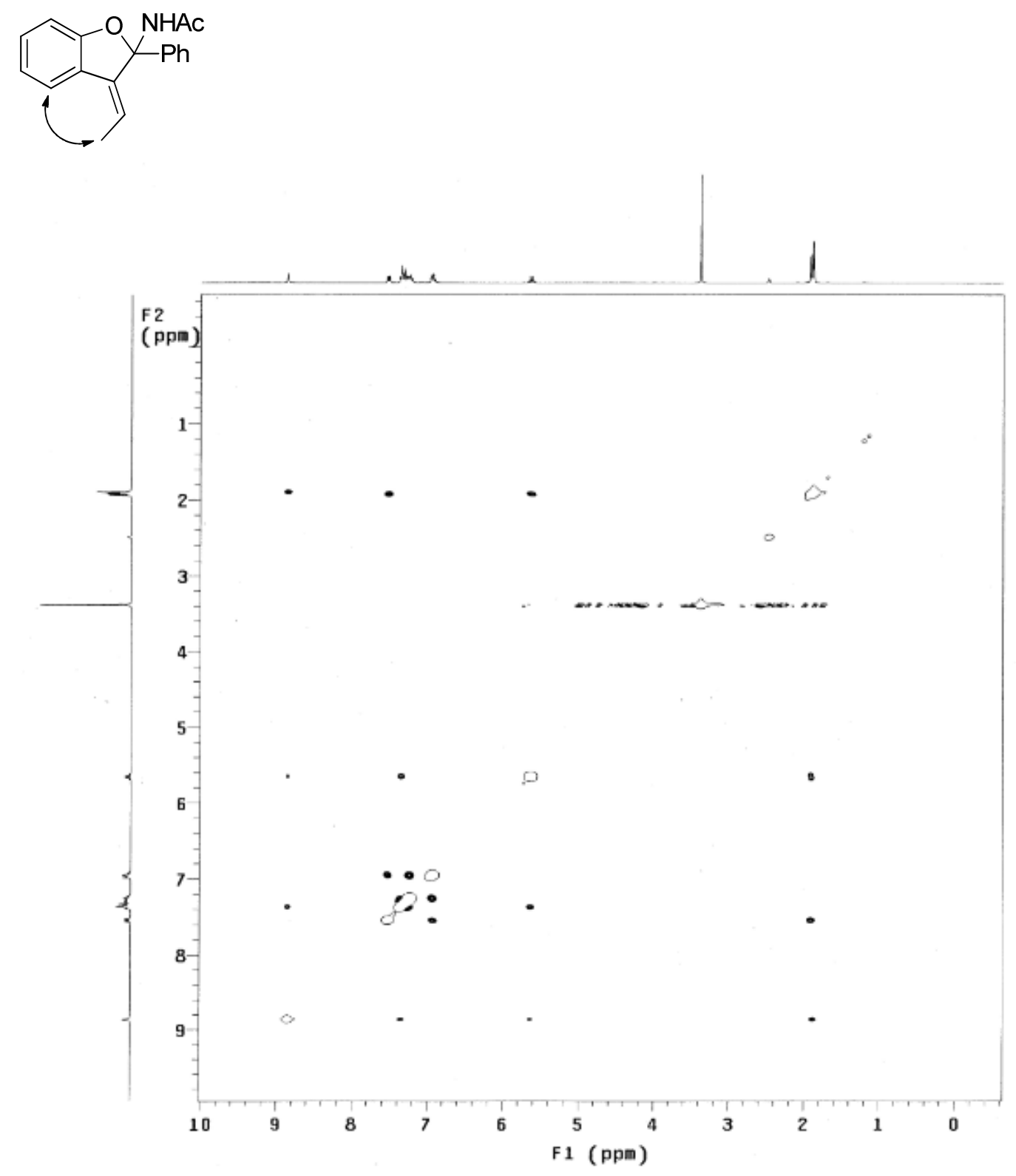



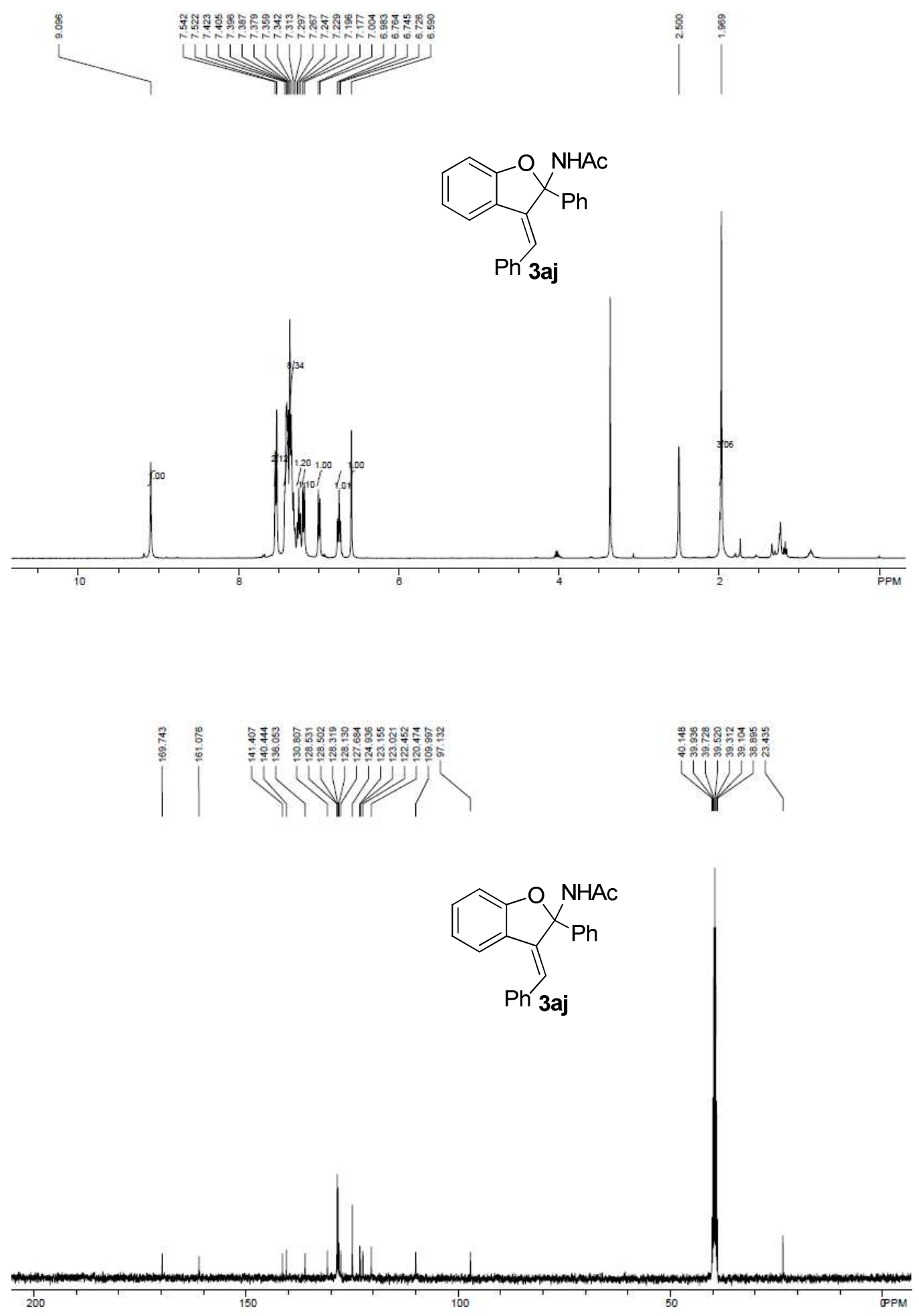

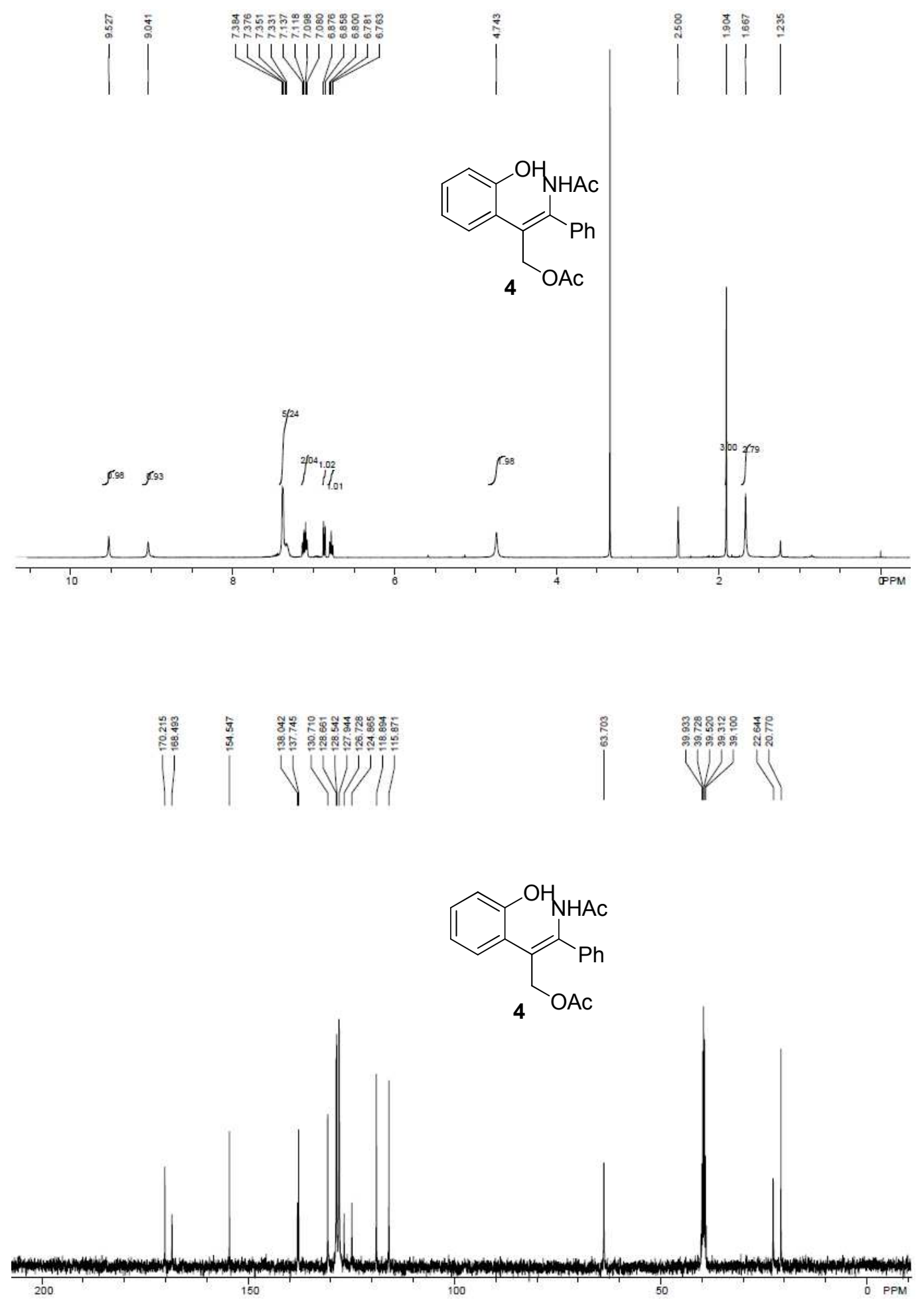


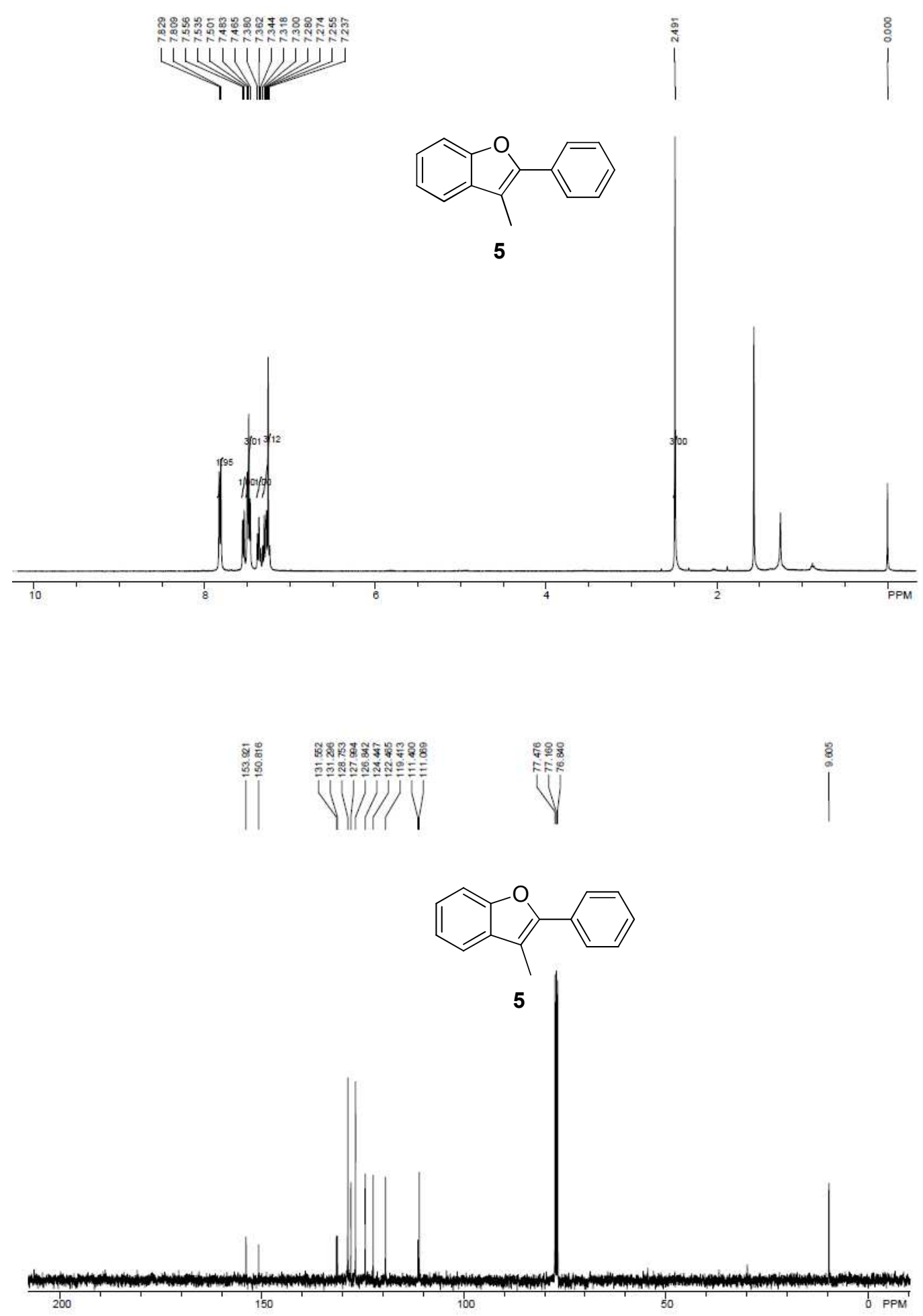



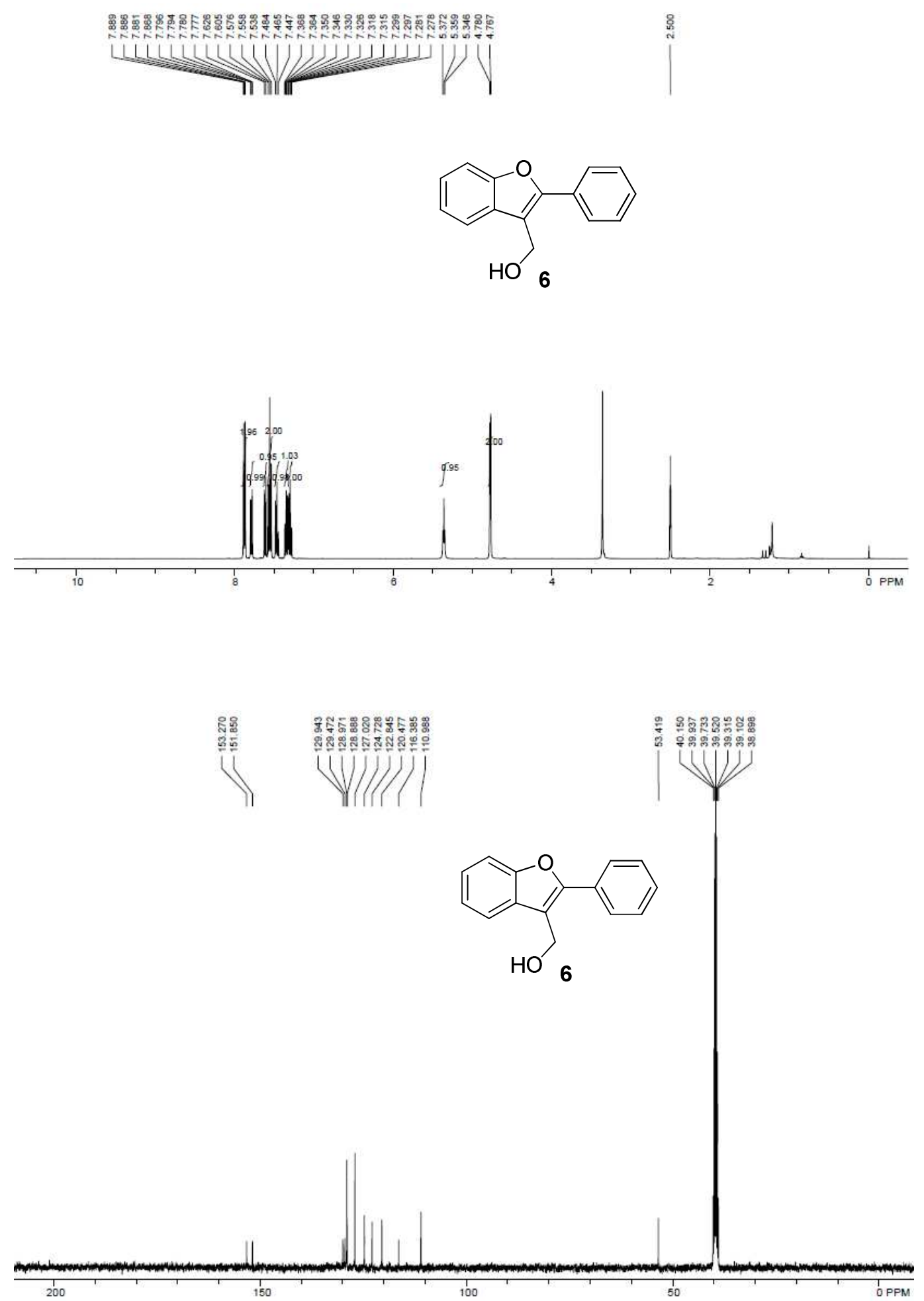

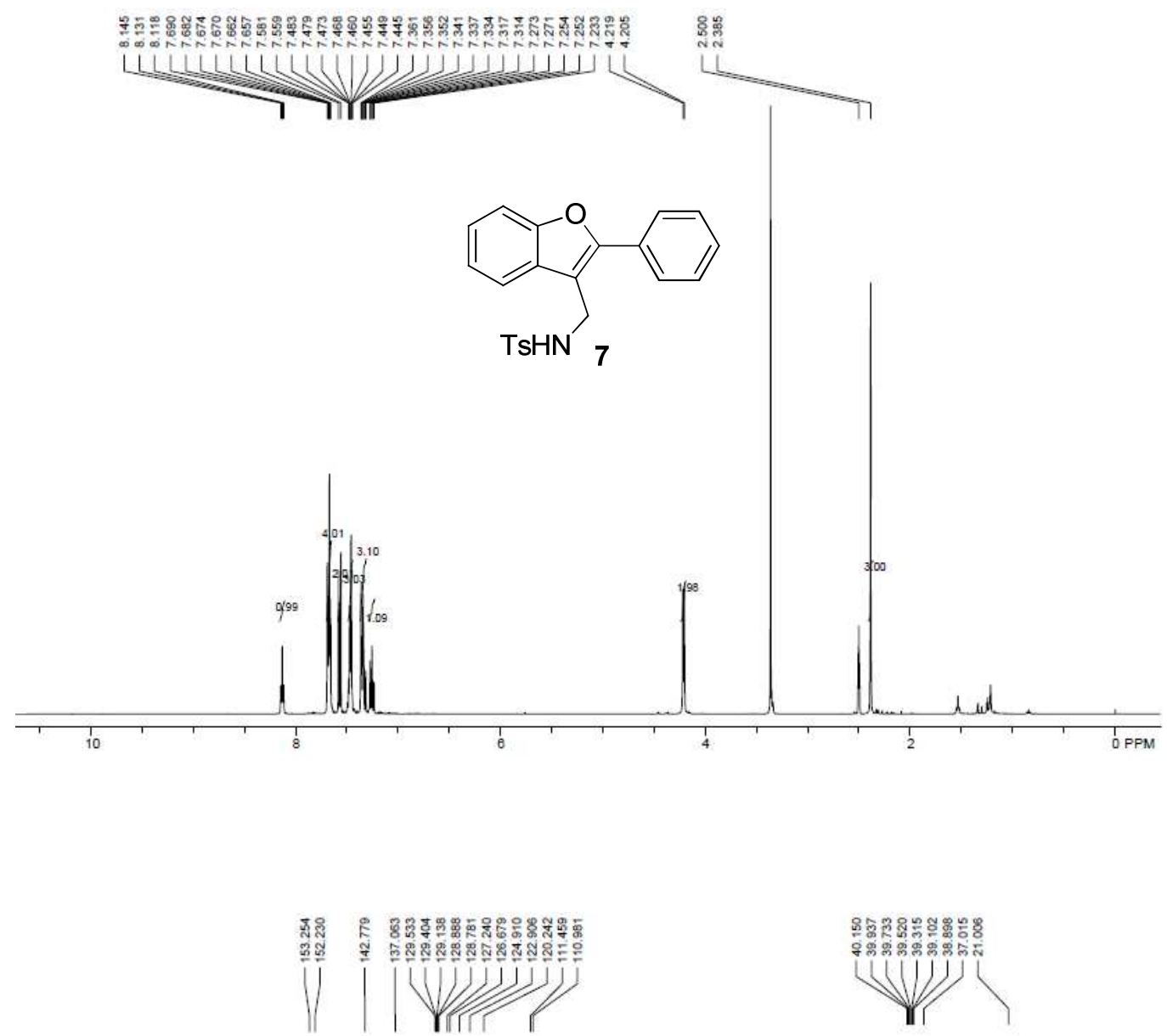

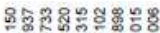

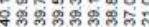

WU
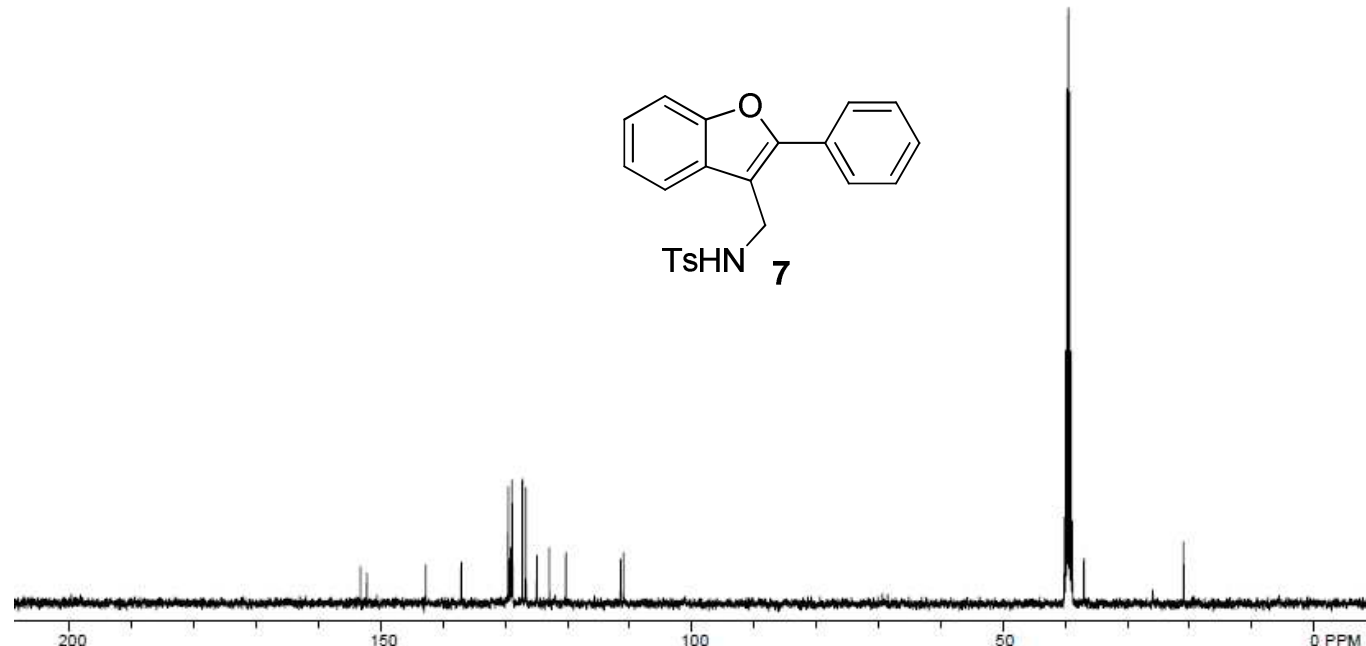

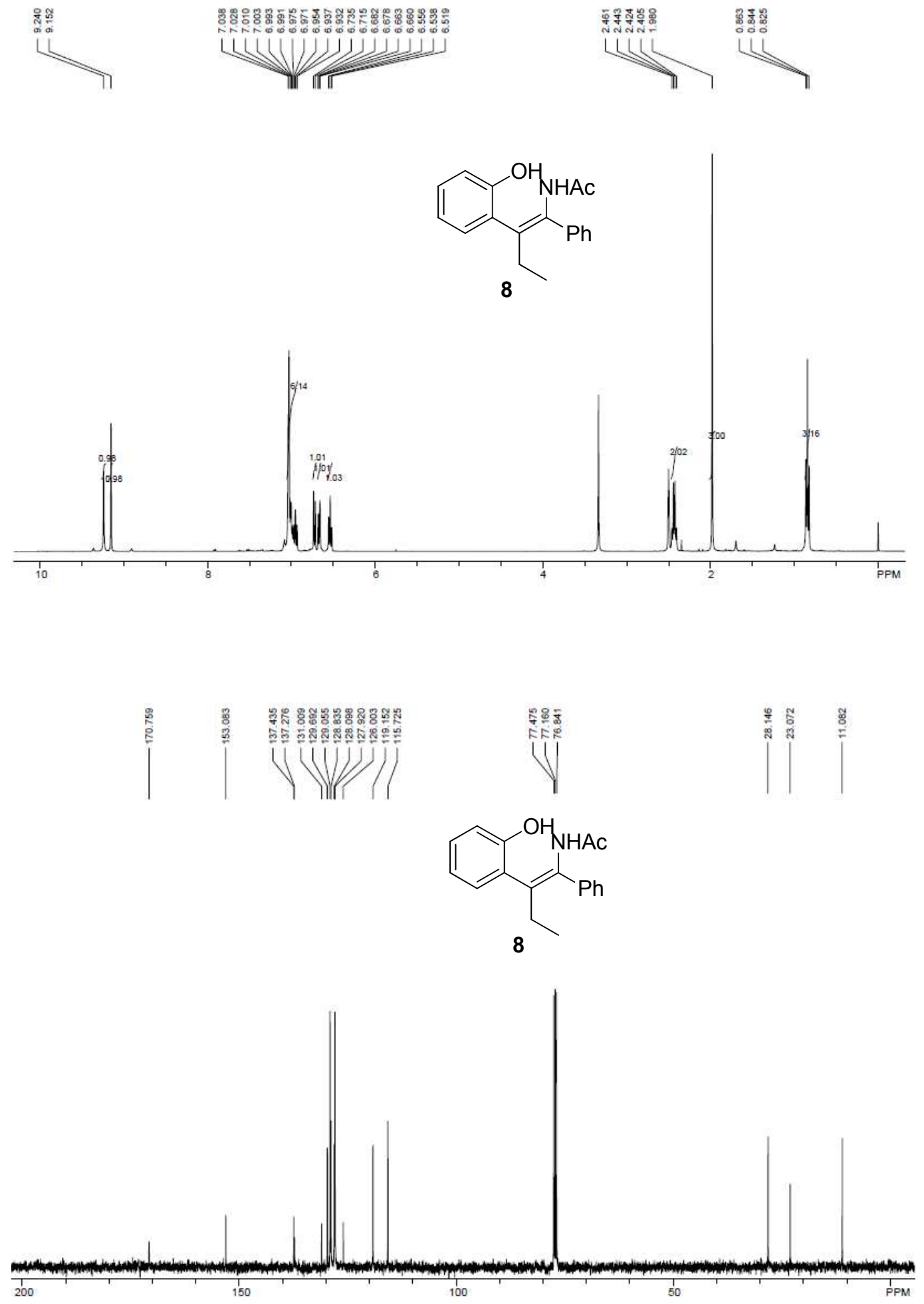

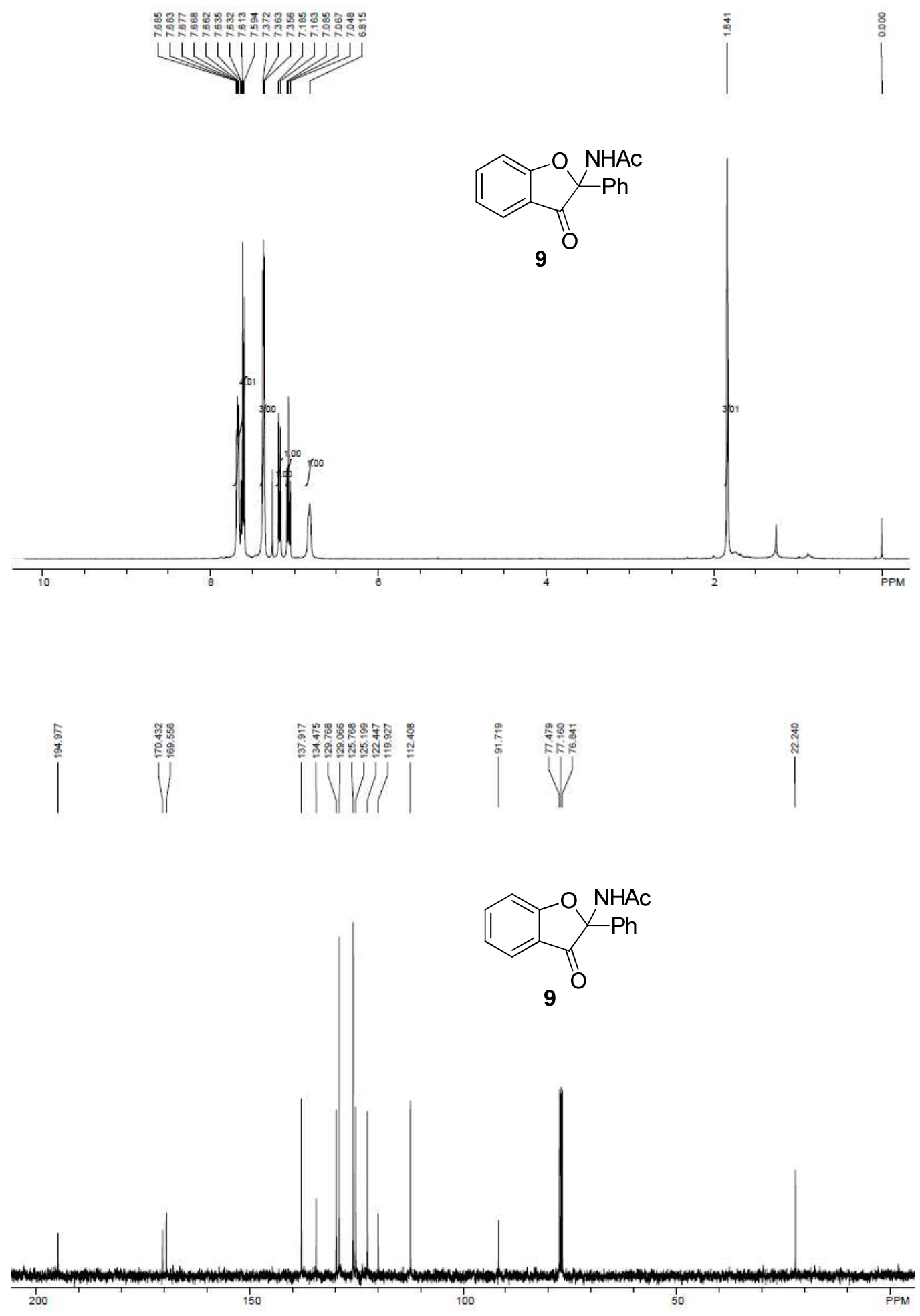